\title{
Supporting information: Changes in filament microstructures during direct ink writing with yield stress fluid support
}

\author{
Leanne Friedrich and Matthew Begley* \\ Materials Department, University of California Santa Barbara, Santa Barbara, CA, 93106, USA. Tel: (805)-679-1122; E-mail: \\ begley@engr.ucsb.edu
}

\section{Contents}

\begin{tabular}{lc}
\hline S1 Stokes numbers & S1
\end{tabular}

\begin{tabular}{ll}
\hline S2 Hypotheses & S2
\end{tabular}

S2.1 Plastic zone flow and disturbed zone flow $\ldots \ldots \ldots \ldots \ldots \ldots \ldots \ldots$ S2

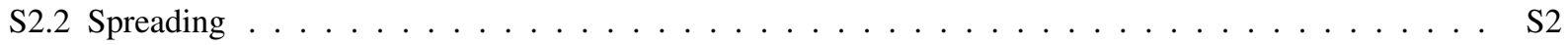

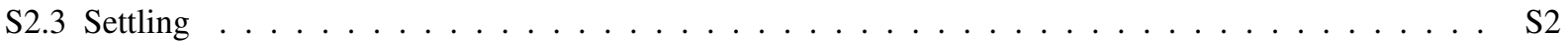

$\begin{array}{lc}\text { S3 Results } & \text { S4 }\end{array}$

S3.1 Correlations between printing parameters and dependent variables $\ldots \ldots \ldots \ldots \ldots$. . . . . . 4

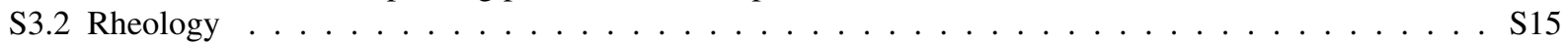

S3.3 Spreading time scales . . . . . . . . . . . . . . . . . . . . . . S16

S3.4 Correlations between dependent variables $\ldots \ldots \ldots \ldots$. . . . . . . . . . . . . . . S19

\begin{tabular}{lr}
\hline S4 Supplemental references & S40
\end{tabular}

\section{S1. Stokes numbers}

A particle can be assumed to follow fluid streamlines where the Stokes number is less than 1 . The Stokes number is defined as:

$$
S t=\frac{\rho_{p} d_{p}^{2} U_{c}}{18 \mu_{f} L_{c}}
$$

where $\rho_{p}$ is the particle density $\left(8.96 \mathrm{~kg} / \mathrm{mm}^{3}\right), d_{p}$ is the particle diameter $(0.015 \mathrm{~mm}), U_{c}$ is the characteristic speed (3-12 mm/s), $\mu_{f}$ is the fluid viscosity (10 Pa.s), and $L_{c}$ is the characteristic length scale $(0.6 \mathrm{~mm}$ nozzle outer diameter).[1] Stokes numbers in this experiment range from 0.056 to 0.224 , indicating that the particles follow fluid flow streamlines. 


\section{S2. Hypotheses}

\section{S2.1. Plastic zone flow and disturbed zone flow}

Table S1: Critical viscoplasticity and inertia parameters for fluids studied in this experiment. $\tau_{y}, \mathrm{~K}$, and $\mathrm{n}$ are fitting parameters for the Herschel Bulkley model, $\rho$ is the fluid density, Od is the Oldroyd number, Re is the Reynolds number, and speeds $(3 \mathrm{~mm} / \mathrm{s}, 12 \mathrm{~mm} / \mathrm{s})$ refer to printing speeds.

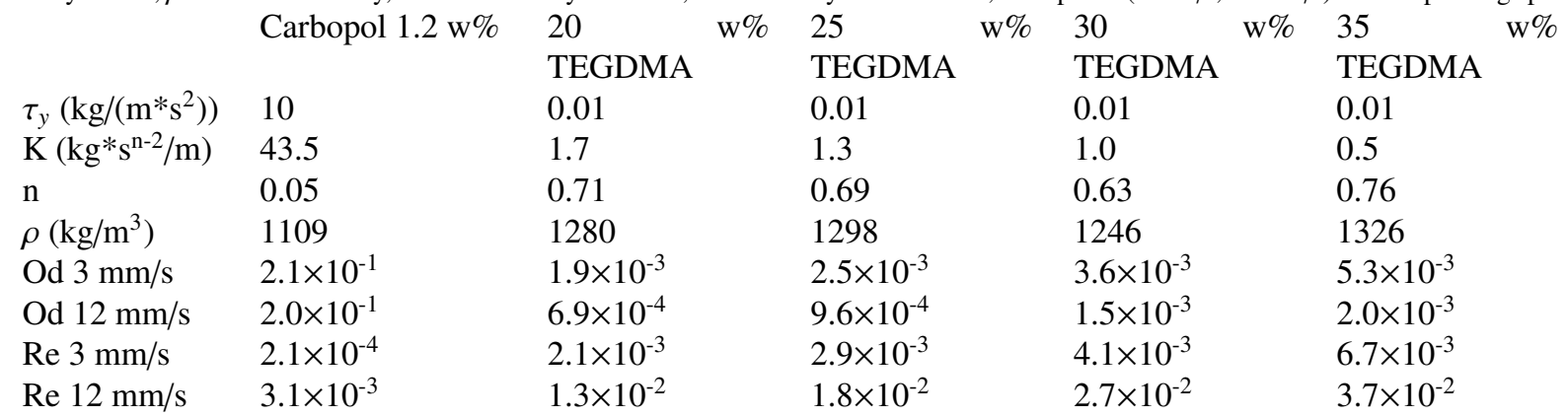

\section{S2.2. Spreading}

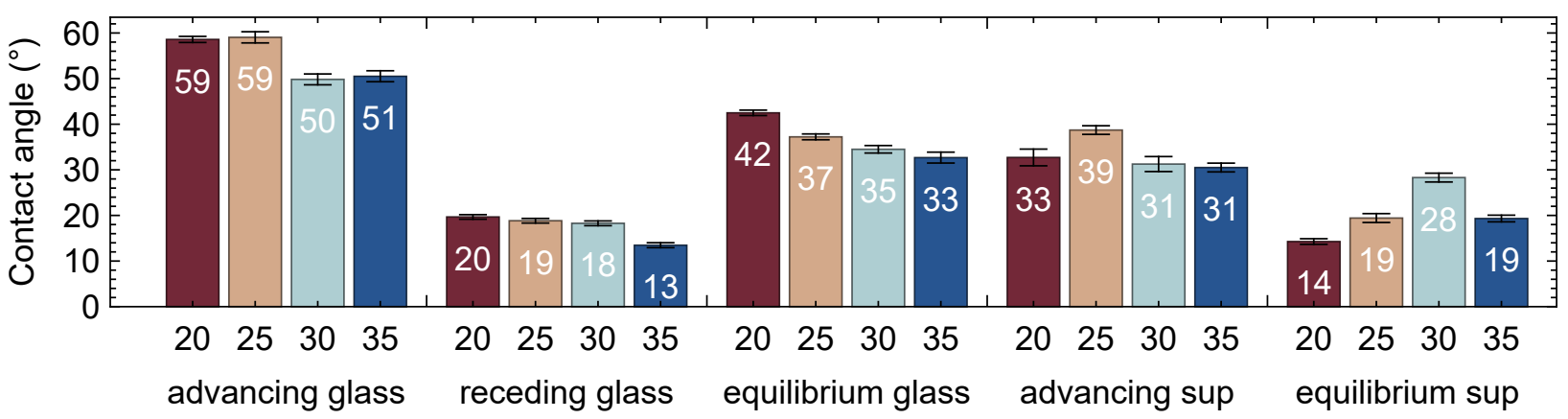

Figure S1: Advancing, receding, and equilibrium contact angles of the inks used in this study, as a function of TEGDMA w\% (20, 25, 30, 35), on glass and a thin layer of $1.2 \mathrm{w} \%$ Carbopol support (sup). Angles were measured using the submerged needle technique. Receding angles on support could not be collected without inducing mixing between the support and ink.

Table S2: Capillary length, which is the critical length scale at which gravity has a non-negligible contribution to the shape of a droplet sitting on a substrate, and Bond number, which a dimensionless metric of the trade-off between gravity and capillarity. Surface tensions were estimated from [2] and [3].

\begin{tabular}{|c|c|c|c|c|c|}
\hline & Carbopol $1.2 \mathrm{w} \%$ & $20 \quad \mathrm{w} \%$ & $25 \quad \mathrm{w} \%$ & $30 \quad \mathrm{w} \%$ & $35 \quad \mathrm{w} \%$ \\
\hline & & TEGDMA & TEGDMA & TEGDMA & TEGDMA \\
\hline Density $\left(\mathrm{kg} / \mathrm{m}^{3}\right)$ & 1109 & 1280 & 1298 & 1246 & 1326 \\
\hline $\begin{array}{l}\text { Estimated surface tension } \\
\left(\mathrm{kg} / \mathrm{s}^{2}\right)\end{array}$ & 0.056 & 0.041 & 0.040 & 0.038 & 0.035 \\
\hline Capillary length (mm) & 2.28 & 1.81 & 1.78 & 1.76 & 1.63 \\
\hline Bond number & 0.017 & 0.027 & 0.028 & 0.029 & 0.034 \\
\hline
\end{tabular}

\section{S2.3. Settling}

At the end of the nozzle, the particles are focused into a square region in the center of the nozzle (Fig. S2). Assuming that this microstructure is preserved as the filament is deposited on the substrate, the deposited particles will form a square distribution in the center of the printed line. In order to focus the particles into the center of the line, there must be a positive acoustic contrast between the particles and matrix. Generally, this means that the particles 
are denser than the polymer matrix. This is true for the silver-coated copper spheres and polyurethane-based matrix used in this study. As such, we expect the square distribution of particles in the center of the line to settle towards the substrate, eventually spreading into a wider distribution when they encounter the substrate (Fig. S2).

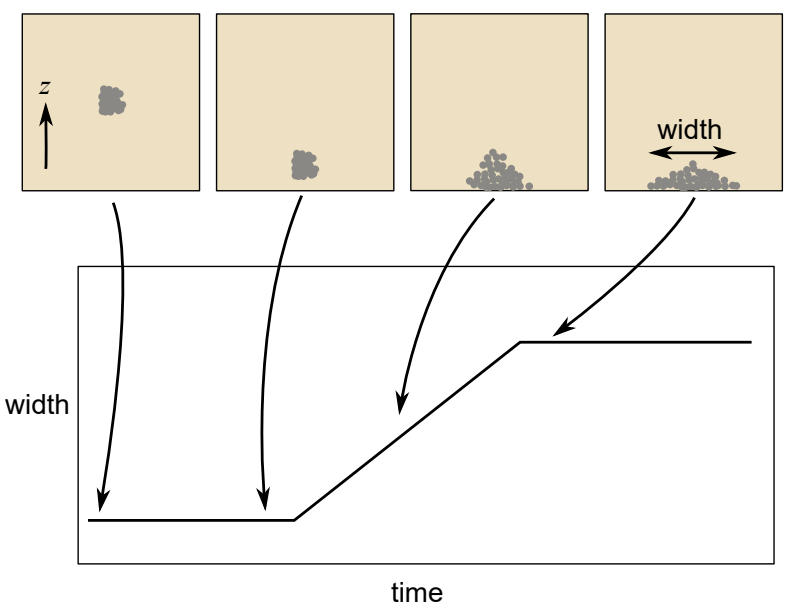

Figure S2: Proposed effect of particle settling on the particle distribution width as a function of time after deposition. Schematics indicate a cross-section of the printed line with particles in gray.

The amount of time that the particles spend settling before reaching the substrate depends on Stokes' drag. Particles falling through the matrix with acceleration due to gravity $g$ achieve a terminal velocity $v$ as a function of the particle density $\rho_{p}$ and fluid density $\rho_{f}$.[4]

$$
v_{g}=\frac{2}{9} \frac{\rho_{p}-\rho_{f}}{\mu} g R^{2}
$$

Using Equation S2, the distance that particles settle during the printing of one layer can be calculated. Because particle volume fractions are low $(\approx 1.4 \mathrm{v} \%)$, Stokes settling should be sufficient, and hindered settling does not need to be considered. The largest printed polygon is an octagon with edge length $12 \mathrm{~mm}$, the smallest polygon is a triangle with edge length $6 \mathrm{~mm}$, and print speeds range from 3-12 mm/s. For $15 \mu \mathrm{m}$ copper microspheres of density 8.96 $\mathrm{g} / \mathrm{cm}^{3}$, we can estimate the critical polygon perimeter for which particles settle to the substrate during one pass as a function of translation speed $v_{s}$, line half-width $w$, and settling speed $v_{g}$.

$$
P_{c}=\frac{w v_{s}}{v_{g}}
$$

Critical perimeters are listed in Table $\mathrm{S3}$

Table S3: Settling of copper microspheres during a single pass around a polygon. The critical perimeter is the polygon perimeter at which particles reach the substrate during a single pass around a polygon.

$\begin{array}{lllll} & 20 \mathrm{w} \% \text { TEGDMA } & 25 \mathrm{w} \% \text { TEGDMA } & 30 \mathrm{w} \% \text { TEGDMA } & 35 \mathrm{w} \% \text { TEGDMA } \\ \text { Density }\left(\mathrm{kg} / \mathrm{m}^{3}\right) & 1280 & 1298 & 1246 & 1326 \\ \text { Viscosity at } 0.01 \mathrm{~Hz}(\mathrm{~kg} /(\mathrm{ms})) & 0.083 & 0.034 & 0.057 & 0.050 \\ \text { Settling velocity }(\mathrm{mm} / \mathrm{s}) & 0.045 & 0.045 & 0.066 & 0.075 \\ \text { Min critical perimeter } P_{c}(\mathrm{~mm}) & 9.9 & 10.1 & 6.8 & 6.0 \\ \text { Max critical perimeter } P_{c}(\mathrm{~mm}) & 39.6 & 40.2 & 27.3 & 23.9\end{array}$

As a reference point, the settling speeds in Table $\mathrm{S3}$ predict that particles should settle out of solution in the ink reservoir on the printer within 20-30 minutes, which is roughly twice as fast as what we observe during experiments. As such, it may be reasonable to double the critical perimeters in Table S3 to more realistic values of 12.0 to $80.4 \mathrm{~mm}$. The polygons printed in this study have perimeters between 18 and $96 \mathrm{~mm}$, so it is realistic to expect that in some tests, the particles will settle to the substrate, and in others, the particles will not reach the substrate. 
From Stokes drag, we expect that higher TEGDMA loadings, which decrease the matrix viscosity, should produce larger changes in the distribution width during relaxation. Printing at faster speeds decreases the amount of time particles have to settle, leading to a smaller initial and relaxed width.

\section{S3. Results}
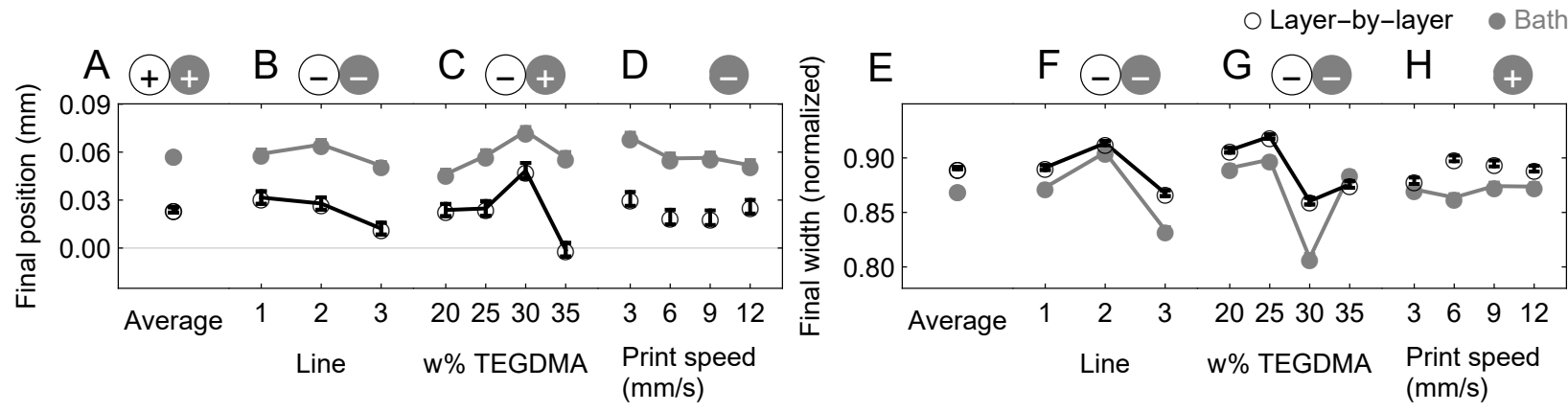

Figure S3: Final particle distribution positions and widths after all lines are printed. Positive positions are inward toward existing lines and the center of the polygon. Distribution widths are normalized by the width of a uniform distribution. Error bars indicate standard error. Signs of statistically significant Kendall $\tau$ parameters are indicated.

\section{S3.1. Correlations between printing parameters and dependent variables}

In this section, we propose a parameter $\tau_{\text {all }}$ which can be used for evaluating the accuracy of hypotheses in multivariable data sets. Altogether, while $\tau_{\text {all }}$ is a useful preliminary metric for evaluating the probability that a driving force influences a measured parameter, it struggles to distinguish between overlapping effects and assumes a linear superposition of effects, so it must be considered in the context of other results, not in isolation.

One way to extract the contributions of the hypothesized effects on the particle distribution and flow field is by comparing correlations between printing parameters and dependent variables to hypothesized correlations. In this work, we printed polygons at varying print speeds, where the flow speed is equal to the translation speed; varying TEGDMA concentrations in the ink, where increasing TEGDMA decreases the ink viscosity; and varying boundary conditions via the three passes around the polygon. For each dependent variable (for example, transverse flow velocity ahead of the nozzle), we can measure the sign of the average value and the sign of the Kendall $\tau$ correlation parameter with each independent variable (pass number, TEGDMA concentration, and print speed). These results are shown in Fig. S4 and Fig. S5

In Table S4, Table S5, Table S6, Table S7, and Table S8, we predict these four signs based on trends found in the literature for each of our five driving forces: the plastic zone, disturbed zone, capillary spreading, gravity spreading, and particle settling. For example, we predict that in layer-by-layer support, the average transverse flow velocity in the nearest neighbor will be negative under plastic zone flow, capillary spreading and gravity spreading, and positive under disturbed zone flow and particle settling. 
Position (mm)
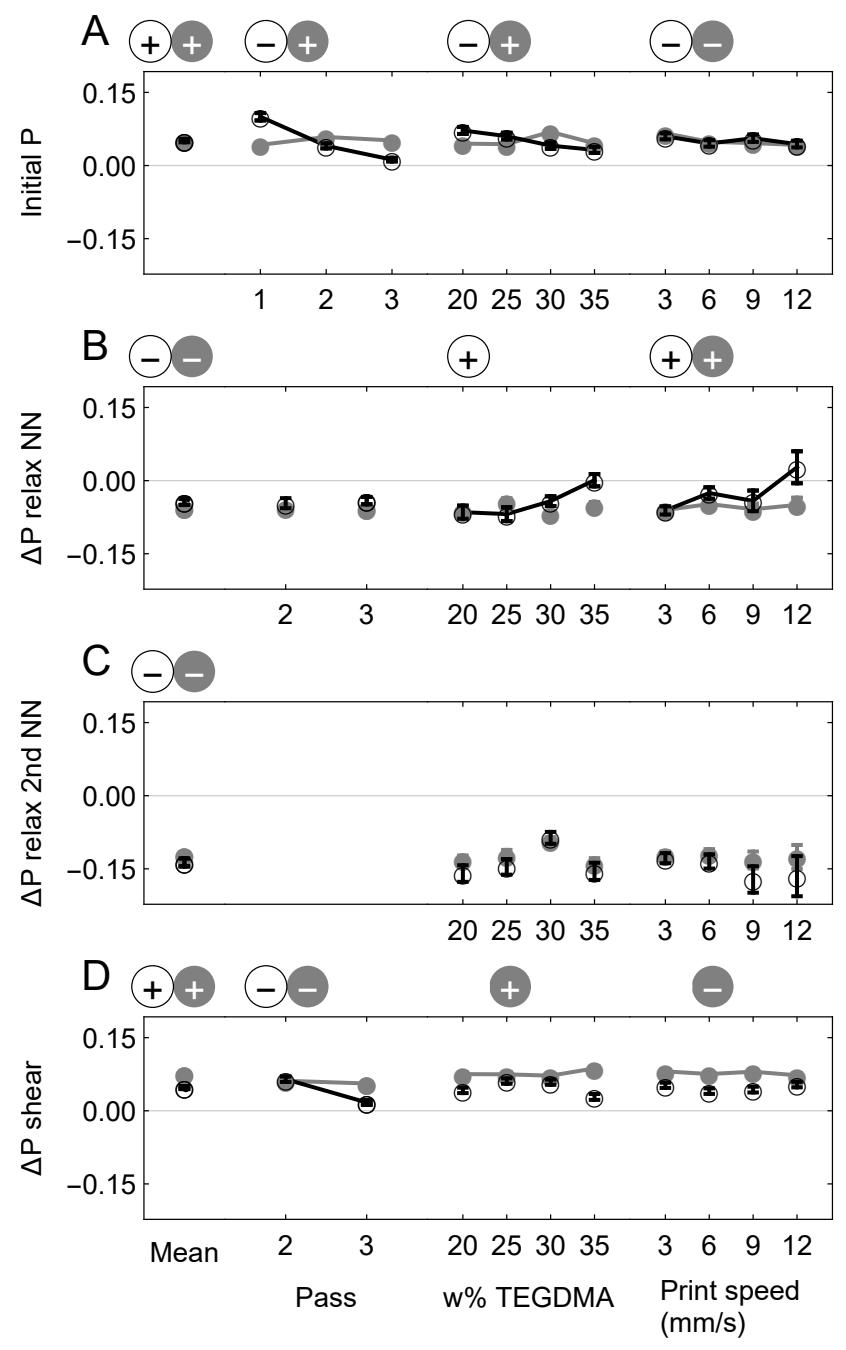

$\bigcirc$ Layer-by-layer $\bigcirc$ Bath Width (normalized)

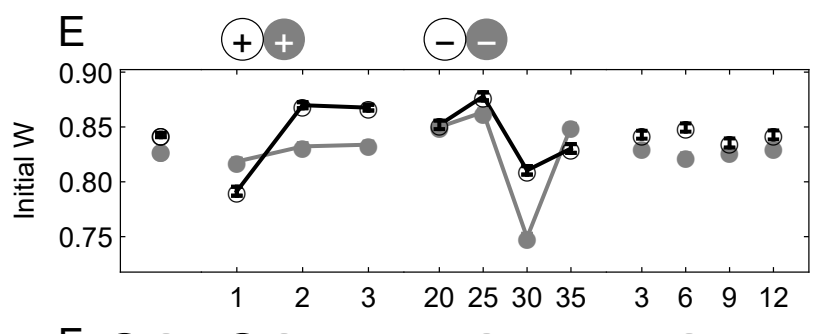

\section{$\mathrm{F} \oplus+\odot$}
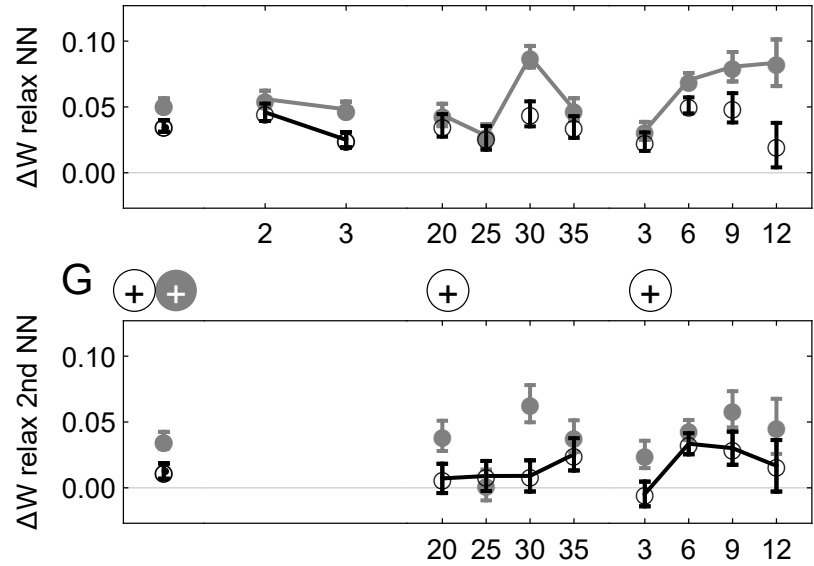

$\mathrm{H} \oplus \odot-\Theta \Theta$

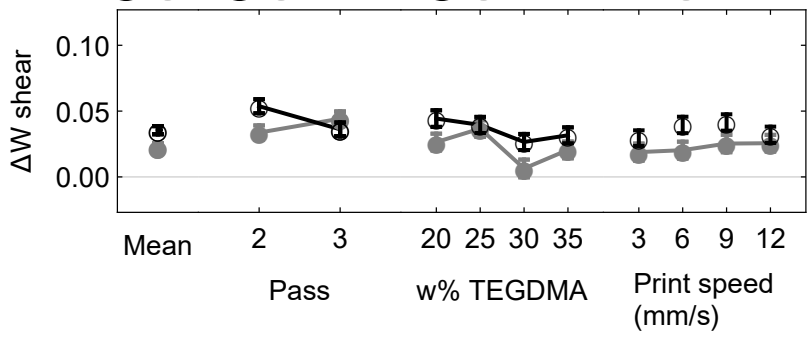

Figure S4: Particle distribution positions (P) and widths (W) as a function of pass number, w\% TEGDMA in the ink, and print speed (the flow speed is equal to the translation speed). Distribution widths are normalized by the width of a uniform distribution. Changes during relaxation are only collected for relaxation times above 7.5 seconds. Error bars indicate standard error. Signs of statistically significant $(p<0.05)$ Kendall $\tau$ parameters are shown. 

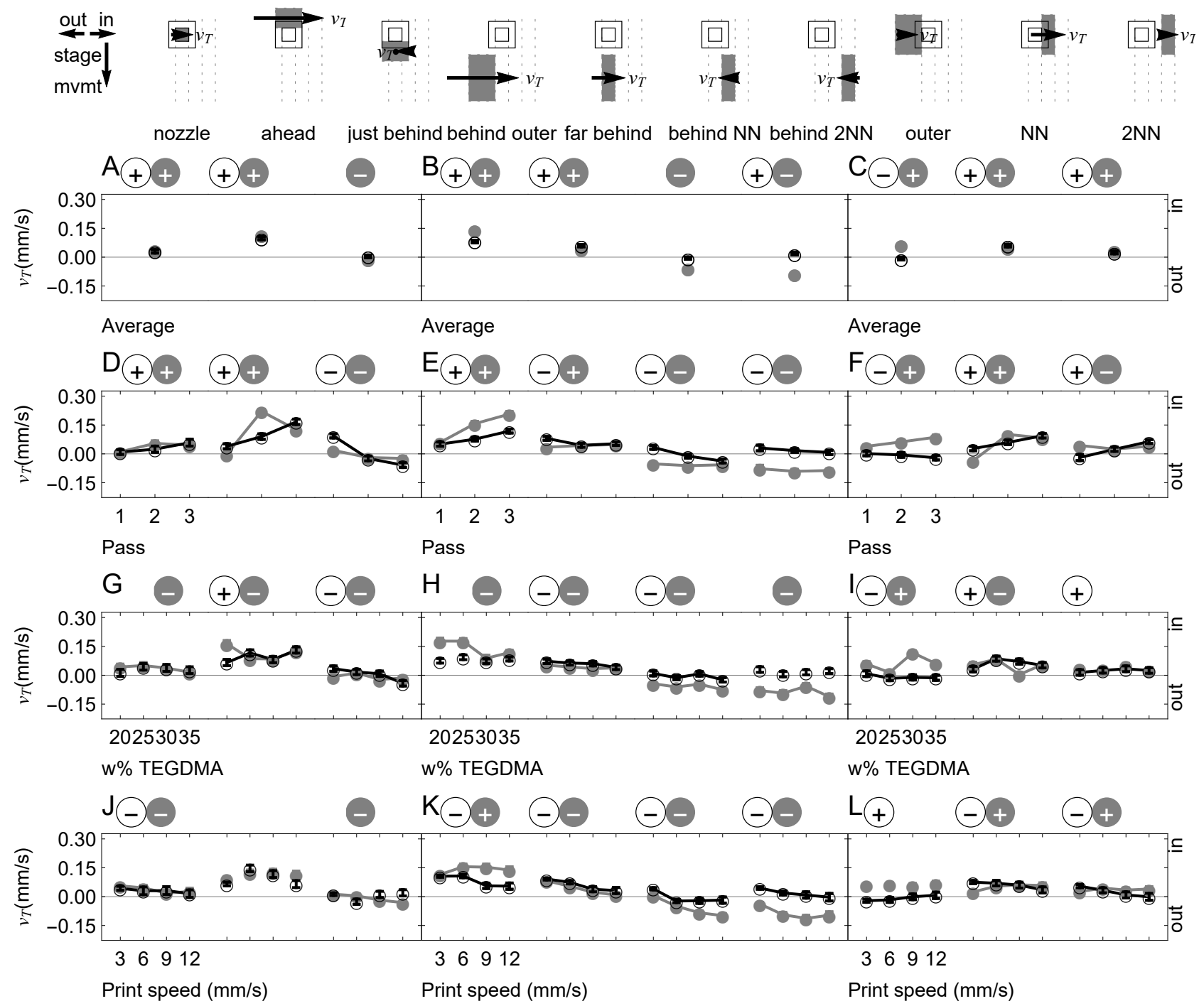

Figure S5: Average transverse flow velocities within ten regions. Positive $v_{T}$ signifies inward flows toward the center of the polygon and nearest neighbors. Signs of statistically significant $(p<0.05)$ Kendall $\tau$ parameters are shown. "NN" nearest neighbor, " $2 \mathrm{NN}$ ' second nearest neighbor. Error bars indicate standard error. 
Table S4: Signs of predicted transverse flows and particle distribution changes due to plastic flow. $\Delta$, pass is the change on successive passes; $\Delta$, TEGDMA is the change with increasing TEGDMA concentration in the ink; $\Delta$, speed is the change with increasing print speed, which is equal to the flow speed and the translation speed. "NN" nearest neighbor, "2NN" second nearest neighbor.

\section{Layer by layer}

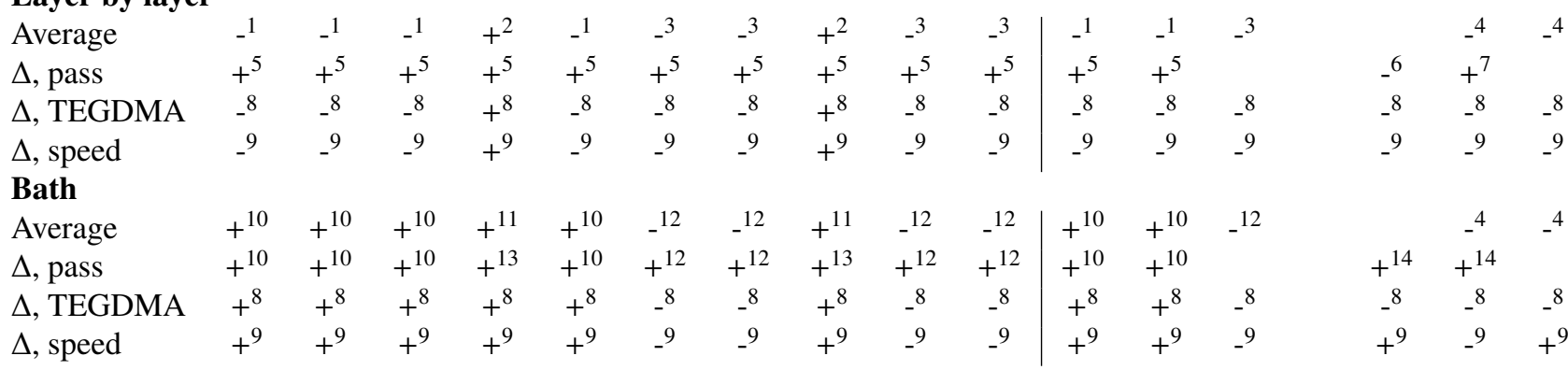

1. On pass 1,2 , and 3, respectively, transverse flows in line with the nozzle are outward, nil, and slightly inward, leading to an average outward (negative) flow

2. On pass 1,2, and 3, respectively, transverse flows in line with the nozzle are nil, nil, and slightly inward, leading to an average inward (positive) flow.

3. On pass 1,2, and 3, respectively, transverse flows in line with the nozzle are outward, nil, and outward, leading to an average outward (negative) flow.

4. Flow of the yielded support material compresses the written ink line.

5. On pass 1, 2, and 3, respectively, transverse flows in line with the nozzle are outward, nil, and slightly inward, leading to an inward (positive) increase in transverse flow velocity with pass number.

6. On successive passes, with decreasing ink viscosity, and with faster speeds, the ink line compresses more because, respectively, the deposited line is more bounded, less resistant to flow, and less resistant to flow due to increased shear rate at fast speeds.

7. Flow of the yielded support material compresses the written ink line a lot less on pass 2, then a little more on pass 3

8. More TEGDMA lowers the viscosity of the ink, enabling more flow.

9. Increasing the translation speed increases the size of the plastic zone.

10. On pass 1,2 , and 3 , respectively, transverse flows in line with the nozzle are nil, slightly inward, and slightly inward, leading to an average inward (positive) flow.

11. Ink flows inward on all passes.

12. Ink in the nearest neighbors shifts outward on the first pass during relaxation, then slightly shifts inward on successive passes.

13. The inner boundary becomes more compliant, and the outer boundary becomes less compliant on successive passes.

14. The ink becomes much less bounded on pass 2 , then only slightly more bounded on pass 3 . 
Table S5: Signs of predicted transverse flows and particle distribution changes due to inertial disturbed zone flow. $\Delta$, pass is the change on successive passes; $\Delta$, TEGDMA is the change with increasing TEGDMA concentration in the ink; $\Delta$, speed is the change with increasing print speed, which is equal to the flow speed and the translation speed. "NN" nearest neighbor, " $2 \mathrm{NN}$ " second nearest neighbor.

Layer by layer

\begin{tabular}{|c|c|c|c|c|c|c|c|c|c|c|c|c|c|c|c|c|c|c|}
\hline Average & -1 & -1 & -1 & $+^{2}$ & -1 & -2 & -2 & -3 & $t^{3}$ & $t^{3}$ & -1 & -1 & -2 & $t^{3}$ & & -2 & + & - \\
\hline$\Delta$, pass & $t^{4}$ & $+^{4}$ & $++^{4}$ & $+^{4}$ & $+^{4}$ & $+^{4}$ & $++^{4}$ & $++^{4}$ & $+^{4}$ & $+{ }^{4}$ & $t^{4}$ & $++^{4}$ & & $+^{4}$ & -5 & -4 & & -4 \\
\hline$\Delta$, TEGDMA & $+{ }^{6}$ & $+{ }^{6}$ & -6 & -6 & -6 & $+^{6}$ & $+{ }^{6}$ & $+{ }^{6}$ & -6 & -6 & $+{ }^{6}$ & -6 & $+{ }^{6}$ & -6 & -6 & -6 & -6 & $+^{6}$ \\
\hline$\Delta$, speed & $+^{6}$ & $++^{6}$ & -6 & -6 & -6 & $+^{6}$ & $+^{6}$ & $+^{6}$ & -6 & -6 & $+^{6}$ & -6 & $+{ }^{6}$ & -6 & -6 & -6 & -6 & $++^{6}$ \\
\hline \multicolumn{19}{|l|}{ Bath } \\
\hline Average & $+^{1}$ & $++^{1}$ & $+{ }^{1}$ & $+^{2}$ & $++^{1}$ & -2 & -2 & -3 & $t^{3}$ & $t^{3}$ & $+^{1}$ & $++^{1}$ & -2 & $t^{3}$ & & -2 & + & - \\
\hline$\Delta$, pass & $+^{4}$ & $++^{4}$ & $+{ }^{4}$ & $++^{4}$ & $t^{4}$ & $++^{4}$ & $+^{4}$ & $++^{4}$ & $+^{4}$ & $++^{4}$ & $t^{4}$ & $+^{4}$ & $+^{4}$ & $+^{4}$ & $+{ }^{4}$ & -4 & & - \\
\hline$\Delta$, TEGDMA & -6 & -6 & $+{ }^{6}$ & -6 & $+{ }^{6}$ & $+^{6}$ & $++^{6}$ & $+^{6}$ & -6 & -6 & -6 & $+^{6}$ & $+^{6}$ & -6 & -6 & -6 & -6 & $+{ }^{6}$ \\
\hline$\Delta$, speed & -6 & -6 & $+{ }^{6}$ & -6 & $+{ }^{6}$ & $+^{6}$ & $++^{6}$ & $++^{6}$ & -6 & -6 & -6 & $++^{6}$ & $+^{6}$ & -6 & -6 & -6 & -6 & $+{ }^{6}$ \\
\hline
\end{tabular}

1. The disturbed zone is larger on the inner edge than the outer edge.

2. The disturbed zone narrows behind the nozzle.

3. The disturbed zone widens at the nozzle.

4. On successive passes, the inner side of the disturbed zone grows due to a more compliant inner boundary, while the outer disturbed zone shrinks due to a less compliant outer boundary.

5. The Oldroyd number decreases on the inner edge by less than it increases on the outer edge.

6. Increasing the TEGDMA concentration in the ink and increasing the print speed elongates and narrows the disturbed zone. 
Table S6: Signs of predicted transverse flows and particle distribution changes due to capillary spreading. $\Delta$, pass is the change on successive passes; $\Delta$, TEGDMA is the change with increasing TEGDMA concentration in the ink; $\Delta$, speed is the change with increasing print speed, which is equal to the flow speed and the translation speed. "NN" nearest neighbor, "2NN" second nearest neighbor.

\section{Layer by layer}

\begin{tabular}{|c|c|c|c|c|c|c|c|c|c|c|c|c|c|c|c|c|c|c|}
\hline Average & -1 & -1 & -1 & -1 & -1 & $-^{2}$ & -2 & -1 & -2 & $-^{2}$ & -1 & -1 & $z^{2}$ & -1 & $t^{3}$ & $t^{3}$ & $+^{4}$ & $t^{3}$ \\
\hline$\Delta$, pass & $+^{5}$ & $+^{5}$ & $+^{5}$ & $+^{5}$ & $+^{5}$ & $+^{5}$ & $+^{5}$ & $+^{5}$ & $+^{5}$ & $+^{5}$ & $+^{5}$ & $+^{5}$ & & $+^{5}$ & $+^{5}$ & $+^{5}$ & & $+^{5}$ \\
\hline$\Delta$, TEGDMA & -6 & -6 & -6 & -6 & -6 & -6 & -6 & -6 & -6 & -6 & -6 & -6 & -6 & -6 & $+^{6}$ & $+^{6}$ & $+^{6}$ & $+^{6}$ \\
\hline$\Delta$, speed & -7 & -7 & -7 & -7 & -7 & -7 & -7 & -7 & -7 & -7 & -7 & $+^{8}$ & $+^{8}$ & $-^{7}$ & +7 & $\_8$ & -8 & $+^{7}$ \\
\hline \multicolumn{19}{|l|}{ Bath } \\
\hline Average & +9 & $+{ }^{9}$ & +9 & $++^{9}$ & $+{ }^{9}$ & $ـ^{2}$ & $-^{2}$ & $++^{9}$ & $ـ^{2}$ & -2 & +9 & $++^{9}$ & $-^{2}$ & $+{ }^{9}$ & $++^{10}$ & $+{ }^{10}$ & $+^{4}$ & $++^{10}$ \\
\hline$\Delta$, pass & $++^{11}$ & $++^{11}$ & $++^{11}$ & $++^{11}$ & $+{ }^{11}$ & -12 & -12 & $++^{11}$ & -12 & -12 & $++^{11}$ & $+{ }^{11}$ & & $++^{11}$ & $+^{5}$ & $+^{5}$ & & $t^{5}$ \\
\hline$\Delta$, TEGDMA & $+^{6}$ & $+^{6}$ & $+^{6}$ & $+^{6}$ & $+^{6}$ & -6 & -6 & $+^{6}$ & -6 & -6 & $+^{6}$ & $+^{6}$ & -6 & $+^{6}$ & $+^{6}$ & $+^{6}$ & $+^{6}$ & $+^{6}$ \\
\hline$\Delta$, speed & $+^{7}$ & +7 & $+^{7}$ & $+^{7}$ & $++^{7}$ & - 7 & -7 & $++^{7}$ & .7 & - 7 & $++^{7}$ & -8 & $+{ }^{8}$ & $+^{7}$ & $++^{6}$ & -8 & -8 & $++^{6}$ \\
\hline
\end{tabular}

1. The transverse component of the contact line velocity is greater on glass than on support because the support is sloped.

2. Adding another ink line pulls existing ink lines outwards (negative).

3. The contact lines travel in opposite directions.

4. Only the outer edge of the nearest neighbor is pulled outward.

5. Ink wets ink more strongly than it wets support, so the existing lines on later passes pull the new line inward (positive) more strongly than the support did.

6. Higher TEGDMA concentrations decrease the viscosity of the ink, decreasing its resistance to flow.

7. Faster flow speeds increase the shear strain rate on the ink and decrease its viscosity, decreasing its resistance to flow.

8. Faster translation speeds decrease the relaxation time, decreasing the change during relaxation.

9. Because the support boundary conditions are symmetric, the only shift in the new line due to capillarity happens because existing ink pulls the new line inwards.

10. Only the outer edge of the nearest neighbor is pulled outward. The other contact lines stay in place.

11. On successive passes, more ink-ink contact lines are introduced, increasing the inward pull on the new line.

12. On successive passes, more ink-ink contact lines are introduced, increasing the outward pull on the existing line. 
Table S7: Signs of predicted transverse flows due to gravitational spreading. $\Delta$, pass is the change on successive passes; $\Delta$, TEGDMA is the change with increasing TEGDMA concentration in the ink; $\Delta$, speed is the change with increasing print speed, which is equal to the flow speed and the translation speed. "NN" nearest neighbor, "2NN" second nearest neighbor.

Layer by layer

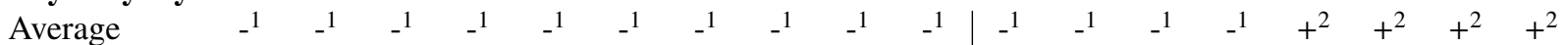

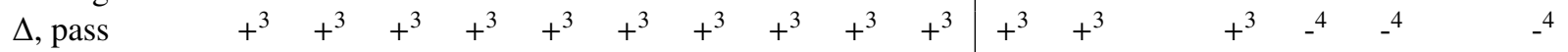

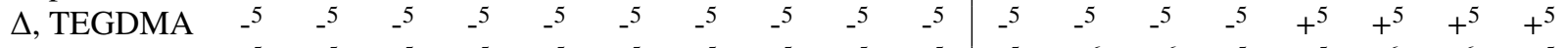

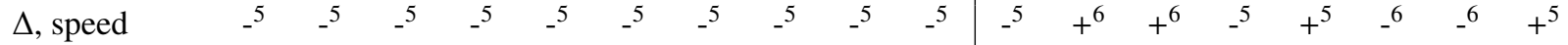

Bath

Average

$\Delta$, pass

$\Delta$, TEGDMA

$\Delta$, speed

1. There is no barrier to spreading on the outer edge.

2. The center of gravity settles lower onto the substrate, widening the line.

3. On pass 1 and 2, the deposited line shifts outward, but on the last pass, the line is bordered on both sides by support, so it does not shift due to gravity.

4. On pass 1 and 2, the deposited line spreads, but on the last pass, the line is bordered on both sides by support, so it does not spread due to gravity.

5. Increasing the TEGDMA content and increasing the print speed decreases the ink viscosity, decreasing its resistance to spreading.

6. Increasing the translation speed decreases the relaxation time, decreasing changes due to relaxation. 
Table S8: Signs of predicted transverse flows and particle distribution changes due to particle settling. $\Delta$, pass is the change on successive passes; $\Delta$, TEGDMA is the change with increasing TEGDMA concentration in the ink; $\Delta$, speed is the change with increasing print speed, which is equal to the flow speed and the translation speed. "NN" nearest neighbor, " $2 \mathrm{NN}$ " second nearest neighbor.

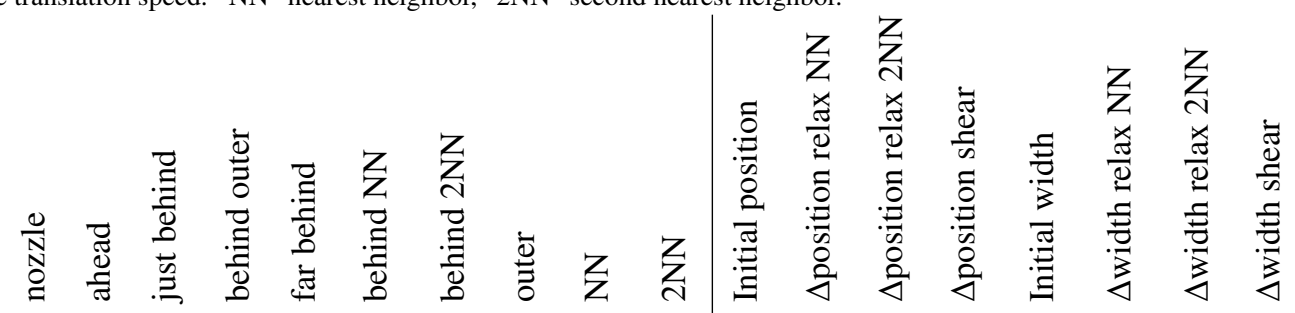

\section{Layer by layer}

Average

$\Delta$, pass

$\Delta$, TEGDMA

$\Delta$, speed

\section{Bath}

Average

$\Delta$, pass

$\Delta$, TEGDMA

$\Delta$, speed
$1 \quad 1$

$\begin{array}{ll}-2 & 1 \\ 4 & \\ -5 & \\ -6 & \end{array}$

$+$

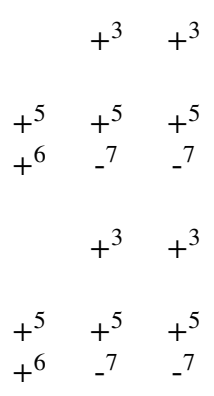

1. Because settling is symmetric, flows in line with the nozzle are net nil.

2. Settling causes the particles to spread outward, resulting in inward (positive) flows inward of the nozzle in the nearest neighbors and outward (negative) flows outward of the nozzle on the left.

3. Settling increases the distribution width.

4. Settling involves movement of particles through the bulk, so boundary conditions do not specifically influence settling.

5. Increasing the TEGDMA concentration decreases the ink viscosity, increasing the terminal velocity of the settling particles.

6. Increasing the flow speed increases the shear rate on the ink, decreasing its viscosity and increasing the terminal velocity of the settling particles.

7. Increasing the translation speed decreases the relaxation time, decreasing changes due to relaxation. 
Table S9: Summary of signs of experimental transverse flows and particle distribution changes, from Figures S4 and S5 $\Delta$, pass is the change on successive passes; $\Delta$, TEGDMA is the change with increasing TEGDMA concentration in the ink; $\Delta$, speed is the change with increasing print speed, which is equal to the flow speed and the translation speed. "-" indicates a negative correlation where $-0.1<\tau<0$, " ++ " indicates a stronger positive correlation where $0.1<\tau<0.2$, and so on, where $-1<\tau<1$. "NN" nearest neighbor, " $2 \mathrm{NN}$ " second nearest neighbor.

\begin{tabular}{|c|c|c|c|c|c|c|c|c|c|c|c|c|c|c|c|c|c|c|}
\hline & $\begin{array}{l}\frac{0}{N} \\
\text { N } \\
\text { ○ }\end{array}$ & $\frac{\widetilde{J}}{\stackrel{\widetilde{J}}{ \pm}}$ & 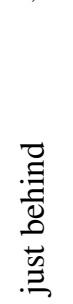 & 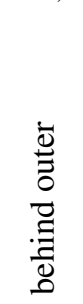 & 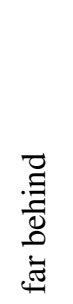 & 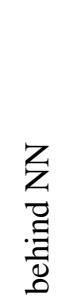 & 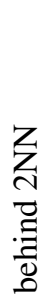 & $\begin{array}{l}\stackrel{\bar{\Xi}}{\Xi} \\
0\end{array}$ & Z & Z & 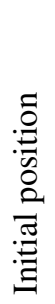 & 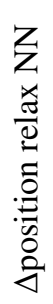 & 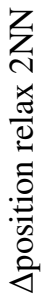 & 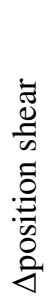 & 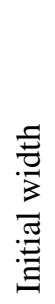 & 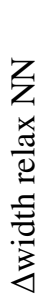 & 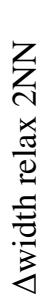 & 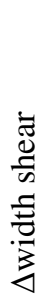 \\
\hline \multicolumn{19}{|c|}{ Layer by layer } \\
\hline Average & + & + & & + & + & & + & - & + & + & + & - & - & + & & + & + & + \\
\hline$\Delta$, pass & + & ++ & --- & ++ & - & -- & - & - & ++ & ++ & - & & & - & ++ & - & & - \\
\hline$\Delta$, TEGDMA & & + & - & & - & - & & - & + & + & - & + & & & -- & & + & - \\
\hline \multicolumn{19}{|l|}{ Bath } \\
\hline Average & + & + & - & + & + & - & - & + & + & + & + & - & - & + & & + & + & + \\
\hline$\Delta$, pass & + & +++ & -- & +++ & + & - & - & ++ & +++ & - & + & & & - & + & - & & + \\
\hline$\Delta$, TEGDMA & - & - & - & - & - & - & - & + & - & & + & & & + & - & + & & - \\
\hline$\Delta$, speed & - & & - & + & --- & --- & -- & & + & + & - & + & & - & & + & & + \\
\hline
\end{tabular}

Table $\mathbf{S 9}$ shows the experimental signs of the average value of the dependent variables and the Kendall $\tau$ correlation parameters. For example, in layer-by-layer support, the average transverse flow velocity in the nearest neighbor is positive. Thus, disturbed zone flow and/or particle settling are likely influential in the nearest neighbor because they predict positive average transverse flow velocities in the nearest neighbor. To further refine whether the transverse flow velocity in the nearest neighbor is influenced more by disturbed zone flow or particle settling, we can consider the change in transverse flow velocity with print speed. The transverse flow velocity becomes more negative with increasing speed, which is predicted by plastic zone flow, disturbed zone flow, capillary spreading, and gravity spreading, but not particle settling. Thus, from these two measurements, we can postulate that disturbed zone flow is the most influential effect in the nearest neighbor. To more precisely quantify our confidence that an effect influences an independent variable (transverse flow velocity in a given region, distribution position, distribution width, change in distribution position, or change in distribution width), we can use the following metric $\tau_{\text {all }}$ which runs from -10 to 10 . $\tau_{\text {all }}$ is calculated for each combination of dependent variable and driving force, for example transverse flow velocity in the nearest neighbor and disturbed zone flow. A large positive $\tau_{\text {all }}$ value indicates high confidence that the driving force influences the dependent variable. A large negative $\tau_{\text {all }}$ value indicates high confidence that the driving force does not influence the dependent variable.

$$
\tau_{\text {all }}=\frac{10}{4}\left(\mu_{t} \frac{\mu_{e}}{\mu_{\max }}+\sum_{\text {pass }, T E G D M A, \text { speed }} \tau_{t} \times\left\{\begin{array}{ll}
\frac{\tau_{e}}{\tau_{\max }} & p \leq 0.05 \\
0 & p>0.05
\end{array}\right)\right.
$$

The factor of 10/4 normalizes $\tau_{\text {all }}$ to run from -10 to $10 . \mu_{t}$ is the predicted sign of the independent variable, -1 or $1 . \mu_{e}$ is the experimental average value of the independent variable. $\mu_{\max }$ is the maximum absolute value of $\mu_{e}$ across transverse velocities in all regions, all positions and changes in position, or all changes in width, whichever set the probed independent variable belongs to. Because all widths are positive, the first term is equal to 0 when the dependent variable is initial width. The second term is summed over the independent variables pass number, TEGDMA concentration, and print speed. $\tau_{t}$ is the predicted Kendall $\tau$ parameter for the correlation between the independent variable and the dependent variable, -1 or $1 . \tau_{e}$ is the experimental $\tau$ correlation parameter between the dependent and independent variable. $\tau_{\max }$ is the maximum absolute $\tau_{e}$ achieved in this data set for the given independent variable for all transverse velocities, positions and changes in position, or widths and changes in width, whichever set the probed independent variable belongs to. $p$ is the p-value for the experimental Kendall $\tau$ test.

$\tau_{\text {all }}$ values are shown in Table S10. For metrics with similar behaviors, the $\tau_{\text {all }}$ value is averaged. For example, "behind inner" represents two regions: behind nearest neighbor and behind second nearest neighbor, so the plastic 
Table S10: $\tau_{\text {all }}$ values indicating confidence in the effect of the five proposed effects (plastic zone, etc.) on transverse flows in various regions and changes in the particle distribution over the course of the print. "behind inner" encompasses behind nearest neighbor and behind second nearest neighbor, and "inner" encompasses the nearest neighbor and second nearest neighbor regions. For the particle distributions, both position and width are considered. $\tau_{\text {all }}$ ranges from -10 to 10 , with 10 indicating high confidence in the influence of the effect on transverse flows or particle distributions and -10 indicating high confidence against the effect.

\begin{tabular}{|c|c|c|c|c|c|c|c|c|c|c|c|c|}
\hline & \multicolumn{6}{|c|}{ Transverse flows } & & & \multicolumn{4}{|c|}{ Particle distributions } \\
\hline & $\begin{array}{l}\overrightarrow{\widetilde{J}} \\
\stackrel{\Xi}{\widetilde{J}}\end{array}$ & $\begin{array}{l}\frac{0}{N} \\
\stackrel{N}{\Xi}\end{array}$ & 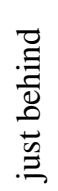 & 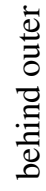 & 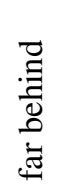 & $\begin{array}{l}\bar{\Xi} \\
. \Xi \\
. \Xi \\
. \Xi\end{array}$ & $\stackrel{\bar{\Xi}}{\stackrel{\Xi}{0}}$ & $\underset{\Xi}{\Xi}$ & : & 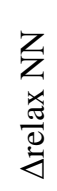 & 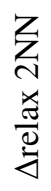 & $\frac{\bar{\Xi}}{\frac{7}{8}}$ \\
\hline \multicolumn{13}{|l|}{ Layer-by-layer } \\
\hline Plastic zone & & -2 & & 1 & 2 & 1 & -1 & 1 & & -4 & -1 & \\
\hline Disturbed zone & -1 & & & 6 & 2 & -4 & & 3 & -3 & -3 & & -2 \\
\hline Capillary spreading & & -2 & & 2 & 2 & 1 & & 1 & & 1 & 1 & -1 \\
\hline Gravity spreading & & -2 & & 2 & 2 & 1 & & 1 & -2 & 2 & 1 & \\
\hline Settling & & & & & & -2 & & & -1 & 1 & & \\
\hline \multicolumn{13}{|l|}{ Bath } \\
\hline Plastic zone & & 3 & -3 & 3 & -2 & 4 & 4 & & 4 & -4 & & \\
\hline Disturbed zone & 3 & 6 & -3 & 7 & -2 & -2 & 2 & 2 & 4 & -2 & 2 & -2 \\
\hline Capillary spreading & & 3 & -3 & 3 & -2 & 4 & 4 & -2 & 2 & -1 & 2 & 2 \\
\hline Gravity spreading & & & & & & & & & & & & \\
\hline Settling & & & & & & -4 & -3 & 1 & -1 & 1 & 1 & \\
\hline
\end{tabular}

zone/behind inner $\tau_{\text {all }}$ value is the average of the $\tau_{\text {all }}$ values for the effect of plastic zone flow on transverse flows in the two regions. A large positive $\tau_{\text {all }}$ indicates high confidence that the theory influences the given metric, while a large negative number indicates high confidence against the theory. For example, in layer-by-layer support disturbed zone flow likely is influential in the behind outer region $\left(\tau_{\text {all }}=6\right)$ but not behind inner $\left(\tau_{\text {all }}=-4\right)$.

Table $\mathrm{S} 10$ can be used to postulate what effects influence transverse flows and particle distributions in different locations and times in the printing process. In some cases, no effects predict the behavior of the system. For example, for transverse flows within the nozzle, no driving forces have positive $\tau_{\text {all }}$ confidence values.

In other cases, the behavior of the system could be explained by many driving forces. For example, in the behind outer region in layer-by-layer support, plastic zone, disturbed zone, capillary spreading, and gravity spreading all have positive $\tau_{\text {all }}$ confidence values. This could be because of overlapping predictions. The magnitude of $\tau_{\text {all }}$ is useful in this case. Disturbed zone flow is more likely than plastic zone flow to influence transverse flow in the behind outer region because it has a much larger $\tau_{\text {all }}$ value.

Far behind the nozzle, all $\tau_{\text {all }}$ parameters are equal to 2 . Care must be taken when considering the meaning of this case. Similar $\tau_{\text {all }}$ values could occur where multiple driving forces predict the same trend. Consider the following example. Force A and force B both predict that the transverse flows increase with TEGDMA concentration. Only force $\mathrm{A}$ is influential in the system, so transverse flows increase with TEGDMA concentration. However, the resultant $\tau_{\text {all }}$ confidence values are the same for force A and force B because they predict the same trend. Alternatively, this could occur where each driving force accurately predicts different trends. Consider the following example. Force A causes transverse flows to strongly scale positively with TEGDMA and weakly scale negatively with print speed, and force B causes transverse flows to weakly scale negatively with TEGDMA and strongly scale positively with print speed. Force A and force B are both influential in the system, so the experimental transverse flow velocity increases with both TEGDMA concentration and print speed. The resultant $\tau_{\text {all }}$ confidence values for force A and force B will be similar because they each accurately predict one experimental trend.

The $\tau_{\text {all }}$ confidence analysis in Table S10 indicates differences in the prominence of plastic zone flow in layer-bylayer and bath support. Confidence values for plastic zone flow are higher in bath support than layer-by-layer support, which could be because bath support requires a larger volume of yielded support material. However, in bath support, evidence of plastic zone flow can be observed inside the nozzle, behind to the sides, and in the outer region, which are largely incongruous regions and may only match plastic zone predictions because of overlap with capillary spreading (Table S10). Likewise, the evidence of plastic zone flow impacting the initial distribution might be due to overlaps with disturbed zone flow and capillary spreading (Table S10]. 


\begin{abstract}
Table S11: Expanded version of Table $\mathbf{S 1 0}$
Transverse flows

Layer-by-layer

Plastic zone

Disturbed zone

Capillary spreading

Gravity spreading

Settling

Bath

Plastic zone

Disturbed zone

Capillary spreading

Gravity spreading

Settling

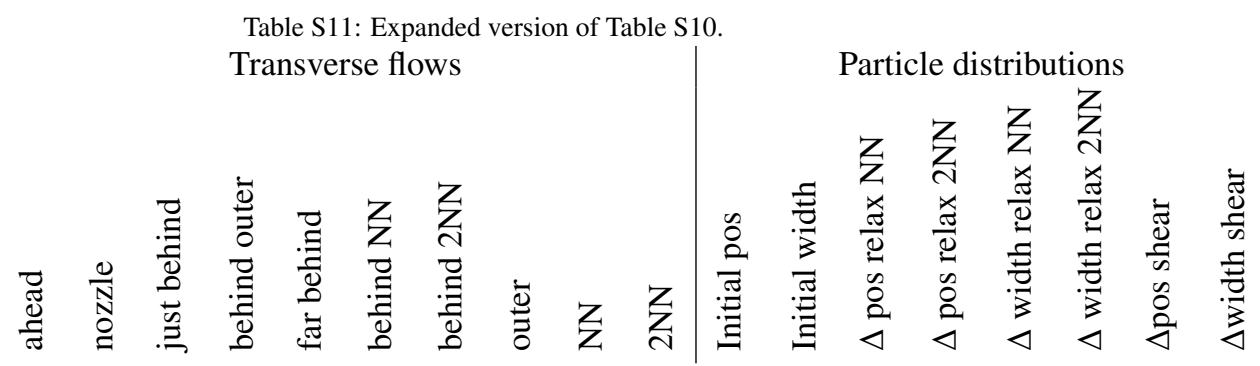

article distributions

\begin{tabular}{ccccccccc|cccccccc} 
& -2 & 1 & 2 & 2 & 1 & -1 & & 2 & 1 & & -4 & 2 & -3 & -5 & & \\
-1 & & 6 & 2 & -5 & -3 & & 3 & 3 & -6 & & -4 & 2 & -2 & -3 & -2 & -2 \\
& & 2 & 2 & 2 & 1 & & & 2 & 1 & & 1 & 2 & 2 & & -3 & 1 \\
& -2 & 2 & 2 & 2 & 1 & & & 2 & 1 & -5 & 1 & 2 & 3 & & -3 & 3 \\
-2 & & & & -3 & -1 & & 1 & -1 & & -2 & & & 2 & & &
\end{tabular}

3

Disturbed zone flow exhibits the highest confidence values of all of the effects, for both layer-by-layer and bath support. Disturbed zone flow is especially prominent in the behind outer region, which makes sense because that region is populated entirely by surplus ink, which would not be subjected to other effects like spreading and shear histories. Otherwise, disturbed zone flow effects can be observed in regions near the nozzle, such as ahead, within the nozzle, in the outer region, and in the inner regions. Disturbed zone flow is the effect that we expect to be most localized to the region near the nozzle, so this agrees with expectation. Disturbed zone flow is also prominent in more regions in bath support, which could be because the larger Oldroyd numbers in bath support compact the disturbed zone into the interrogated zone, while in layer-by-layer support the disturbed zone may be larger than the interrogated zone.[5]

$\tau_{\text {all }}$ confidence values indicate low-level confidence in capillary and gravity spreading for layer-by-layer support and slightly higher confidence in capillary spreading for bath support. It is possible that both types of spreading occur in layer-by-layer support, leading to more diffuse evidence of each spreading force because contact line movement due to gravity and capillarity oppose each other at times. Because we did not predict any effect of gravity spreading in bath support, $\tau_{\text {all }}$ values must be 0 for gravity spreading in bath support. In layer-by-layer support, spreading can be more prominently observed in the particle distribution during relaxation, while in bath support, evidence of spreading can also be observed in the initial distribution and change during shear, which could come from overlap with disturbed zone flow or interactions between effects.
\end{abstract}




\section{S3.2. Rheology}
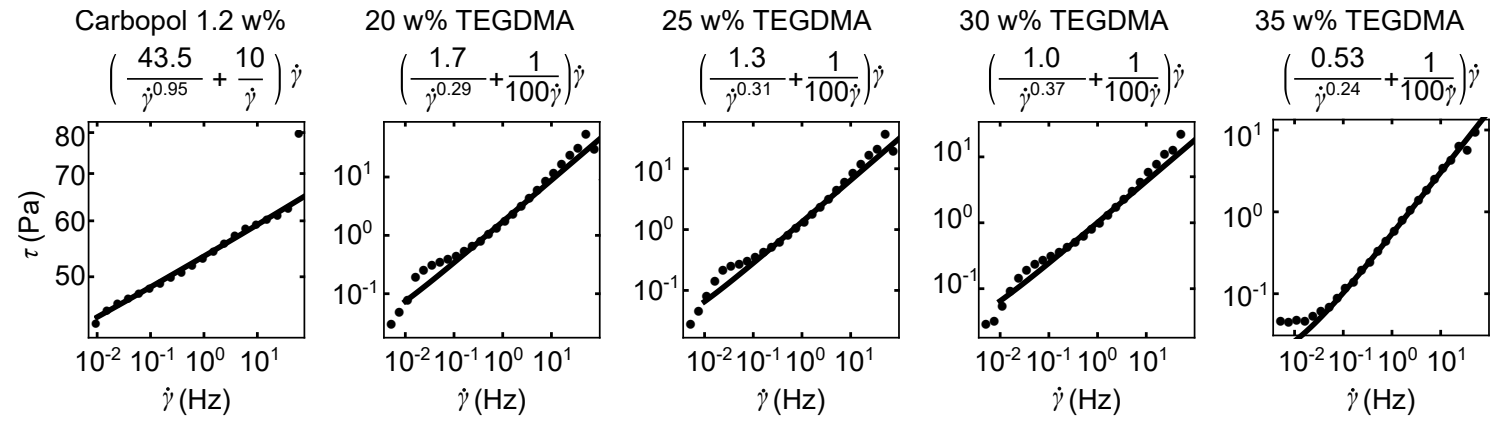

Figure S6: Herschel-Bulkley fits for fluids studied in this experiment. $\tau$ is the shear stress, and $\dot{\gamma}$ is the shear strain rate.

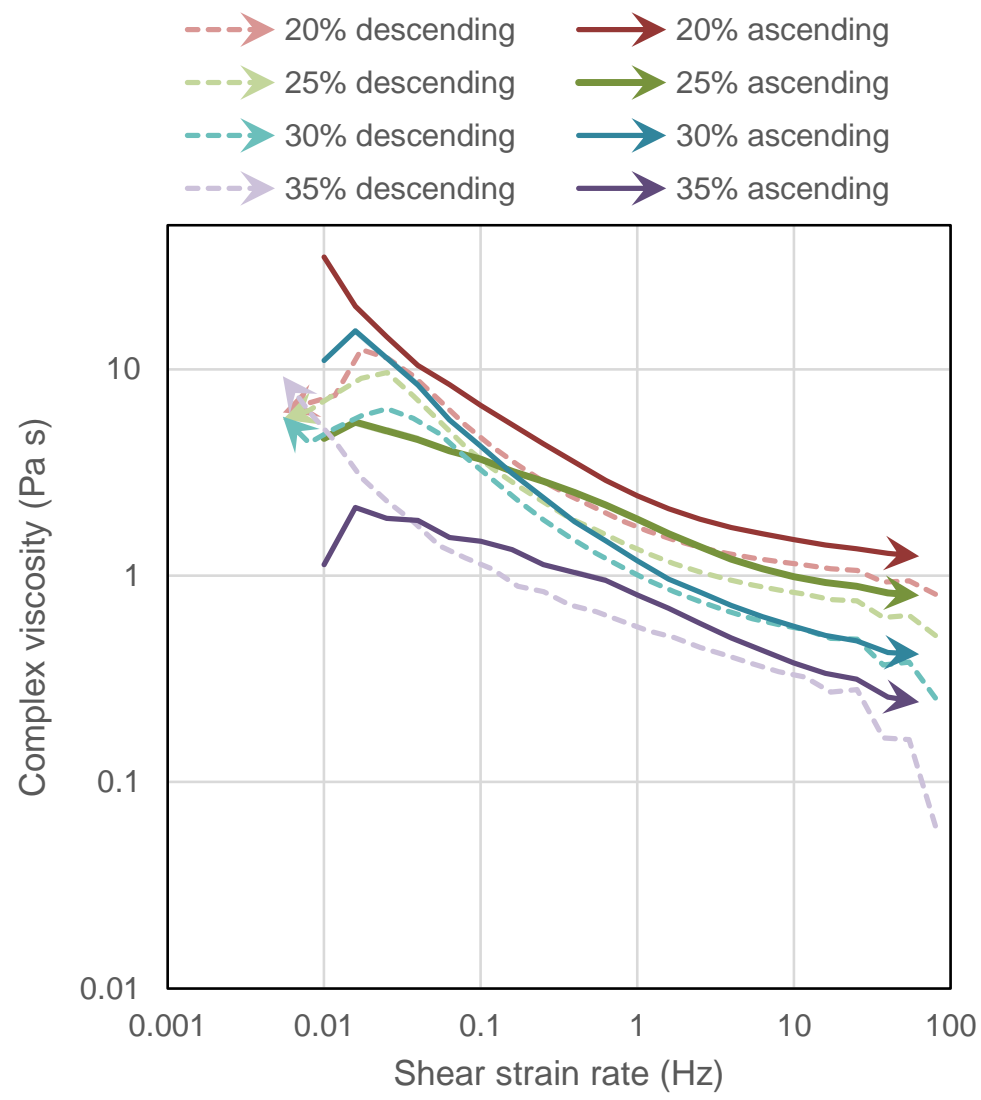

Figure S7: Viscosity of inks as a function of shear strain rate measured during oscillatory strain rate sweeps collected using ascending or descending strain rates, with varying wt $\%$ TEGDMA.

The measured complex viscosity of the inks varies between sweeps collected with ascending and descending strain rates, at very high and very low shear strain rates. Note that at low shear strain rates, the $35 \mathrm{wt} \%$ TEGDMA exhibits an unusually high viscosity during the descending sweep, and the $30 \mathrm{wt} \%$ TEGDMA exhibits an unusually high viscosity during the ascending sweep. These complex rheological behaviors may contribute to the lack of correlation between ink composition and flow behaviors shown in previous sections. 


\section{S3.3. Spreading time scales}

Depending on the desired characteristics of the particle distribution, relaxation can be useful tool or an obstacle. To understand the roots of changes in distribution during relaxation, we can measure the change in distribution as a function of time. The scaling of the change in distribution with time indicates that the observed spreading is more likely to come from capillarity than gravity. Because we printed polygons with a range of perimeters and printing speeds, initial distributions and distributions after relaxation are measured at varying lengths of time apart. Based on capillarity-driven spreading, we would expect the contact line speed to scale with time raised to the $1 / 10$ or $1 / 7$, depending on the model used.[6] Based on gravity-driven spreading, we would expect the power law scaling to be $1 / 5$ or $1 / 8$. [6] Based on a combination of gravity and capillarity, we expect a $1 / 4$ or $1 / 7$ scaling. [6] If we assume that the change in position during relaxation and the change in width during relaxation are proportional to the contact line velocity, the changes in position and width during relaxation as a function of time can be used to evaluate the relative strengths of capillarity and gravity.

Measuring an exact scaling of the spreading velocity in these experiments is not straightforward. Figure $\mathbf{S} 8$ shows the change in position and change in width during relaxation as a function of relaxation time for layer-by-layer and bath support. While long time-scale changes in position are negative, short time-scale changes are positive (Fig. S8A,B). Similarly, while long time-scale changes in width are positive, short time-scale changes are negative (Fig. [S8C,D). If we discard the few positive changes in position, we can fit a linear regression to the log of the change in position $(\Delta p)$ as a function of the log of time $(t)$ to determine the scaling of the change in position with time (Fig. S9]. These fits demonstrate that the change in position scales with time following the relationship $\Delta p \propto t^{1 / 10}$. However, because this fit excludes some of the data set, its relevance is questionable. Instead, it may be more appropriate to assume that the change in position follows the equation $\Delta p=a+b t^{c}$, where $a$ represents some constant positive shift in position that occurs at the point when the relaxed position is measured, possibly because the relaxed position is measured within the disturbed zone or plastic zone. Capping $a$ at $0.6 \mathrm{~mm}$, which is the outer width of the nozzle, the scaling exponent $c$ is on the order of 1/100, which is smaller than any of the models in the literature (Fig. [S8A,B). [6] Similarly, fitting the change in width using the same model, the scaling exponent is still on the order of 1/100 (Fig. S8 C,D). A more accurate fit for both change in width and position can be achieved with the equation $\Delta p=a+b(t-d)^{c}$, assuming that for some period of time $d$ after deposition, the printed line does not relax at all, perhaps because the line is still within the disturbed zone (Fig. S10). With time parameters $d$ near 1 second, this fit still produces scaling exponents on the order of 1/100. An experiment which probes longer relaxation times could produce a more precise scaling relationship between the change in position and relaxation time.

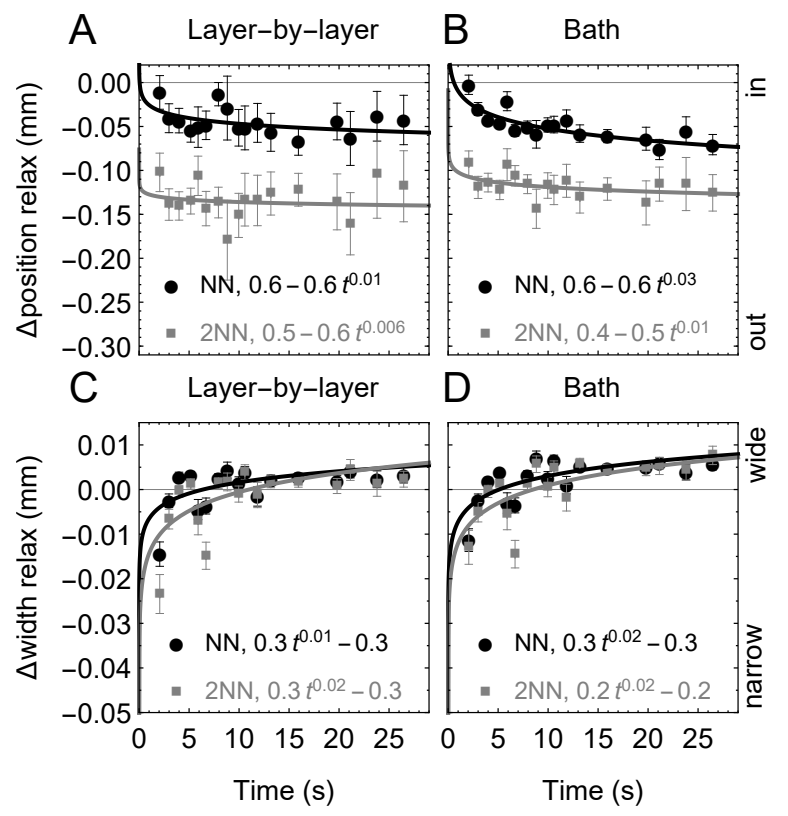

Figure S8: Changes in particle distribution positions and widths during relaxation as a function of time between measurement of the initial and relaxed distributions. Error bars indicate standard error. "NN" nearest neighbor, "2NN" second nearest neighbor. 


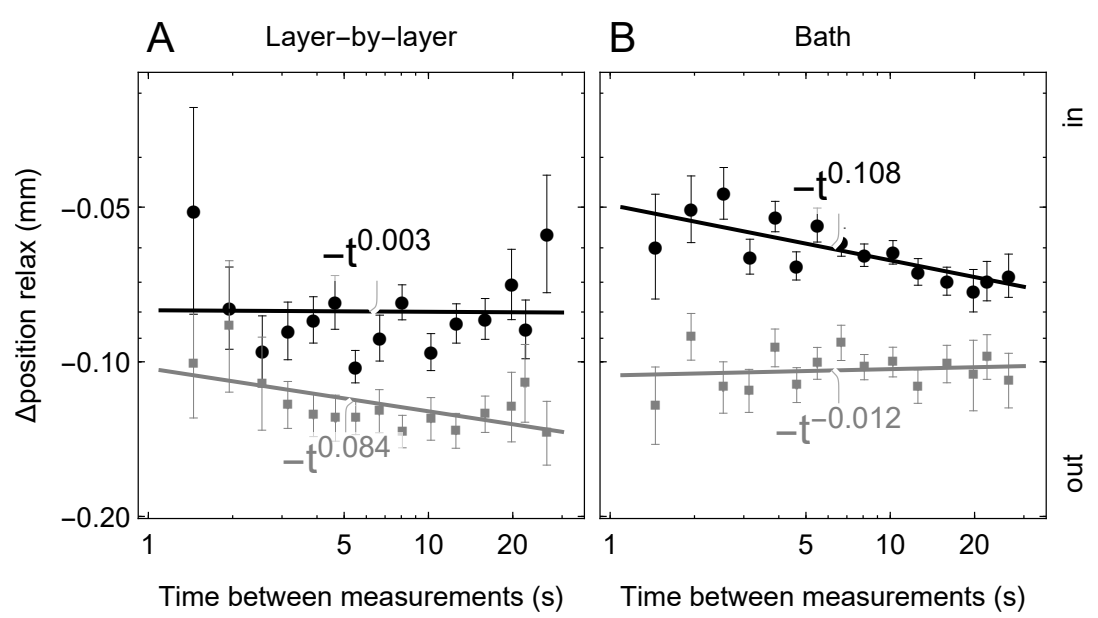

Figure S9: Log-log plot of the negative change in position during relaxation as a function of relaxation time. Positive changes in position are discarded, and averages of logs are shown. 

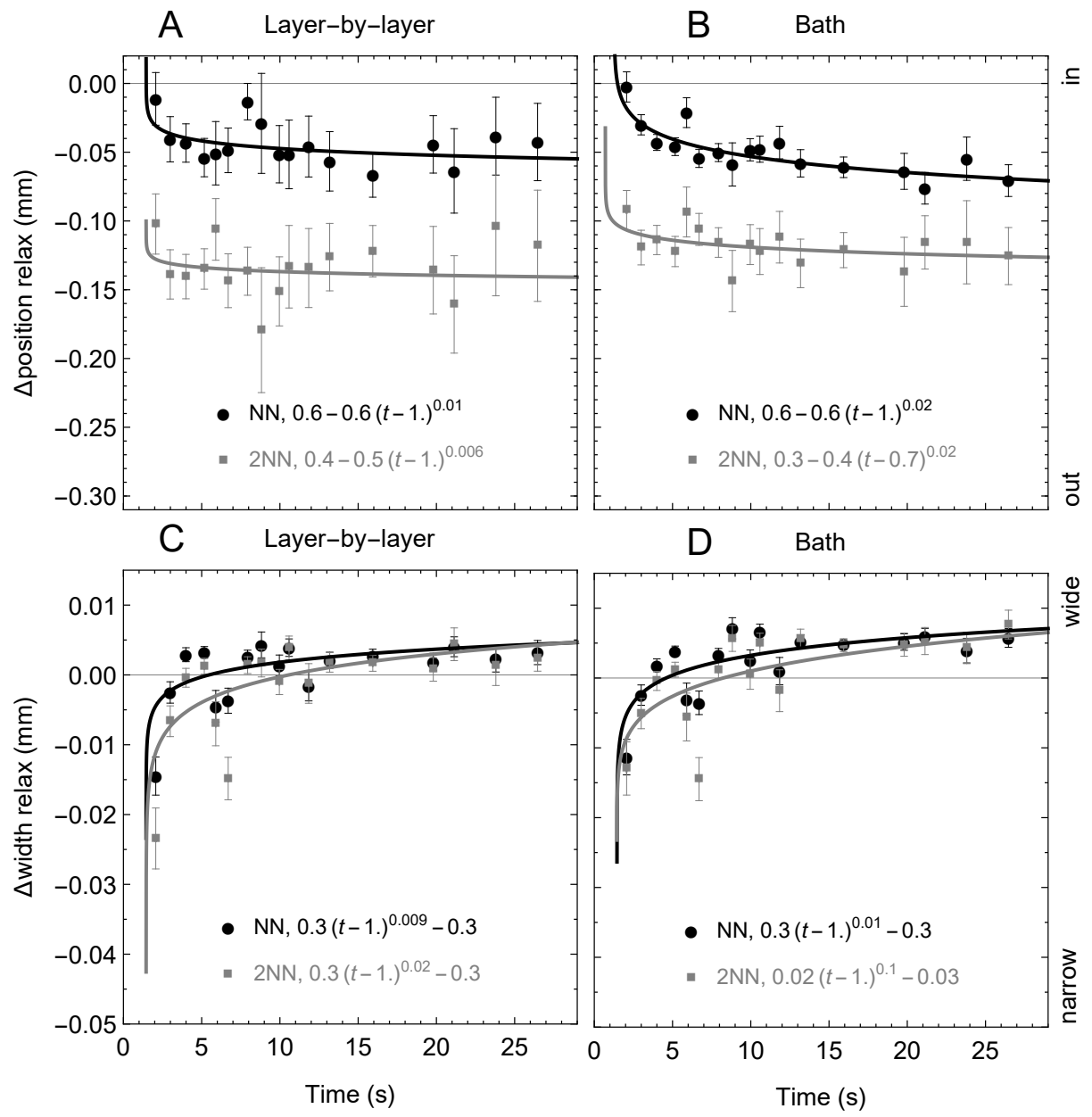

Figure S10: Changes in particle distribution positions and widths during relaxation as a function of time between measurement of the initial and relaxed distributions. Error bars indicate standard error. "NN" nearest neighbor, "2NN" second nearest neighbor. 


\section{S3.4. Correlations between dependent variables}

Table S12: Summary of statistically significant Kendall $\tau$ correlations between absolute particle distribution positions, where $p<0.05$. "-" indicates a negative correlation where $-0.1<\tau<0$, "++" indicates a stronger positive correlation where $0.1<\tau<0.2$, and so on, where $-1<\tau<1$.

\section{Layer-by-layer}

Initial position

Relaxed position NN

Relaxed position 2nd NN

Sheared position

Bath

Initial position

Relaxed position NN

Relaxed position 2nd NN

Sheared position
Initial position Relaxed position NN Relaxed position 2nd NN Sheared position
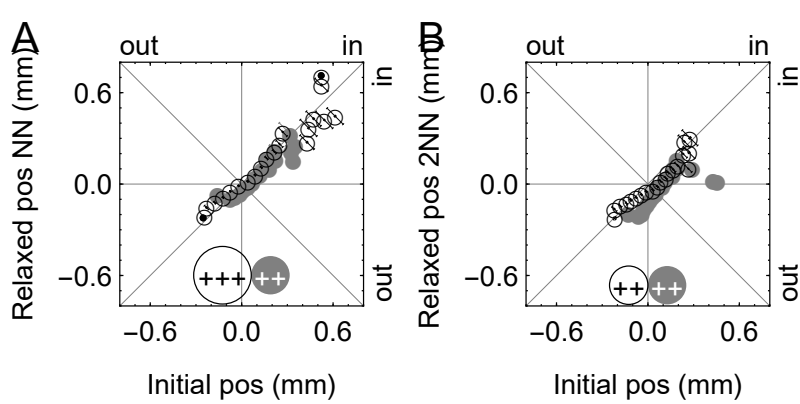

○ Layer-by-layer • Bath
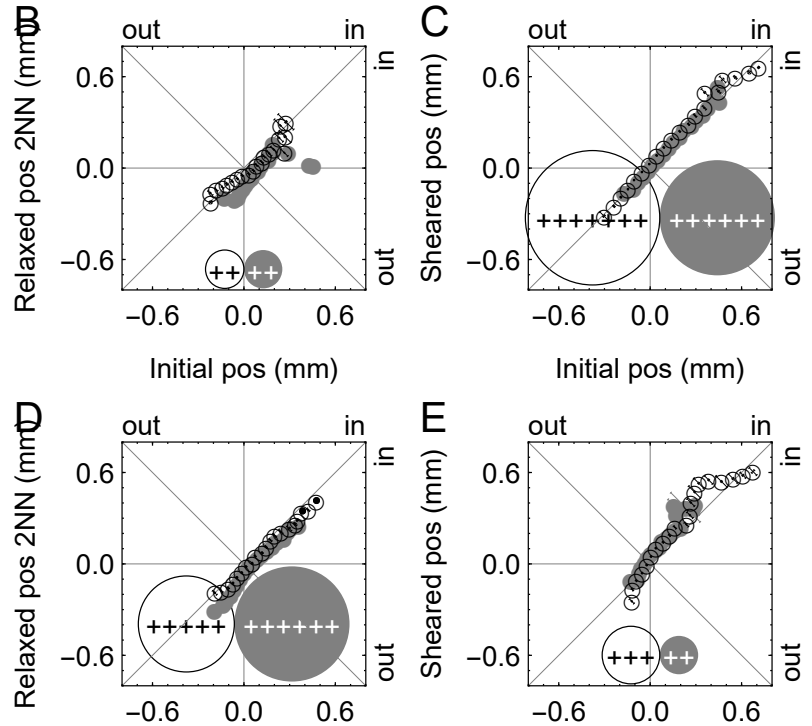

Relaxed pos NN (mm)

Relaxed pos NN (mm)

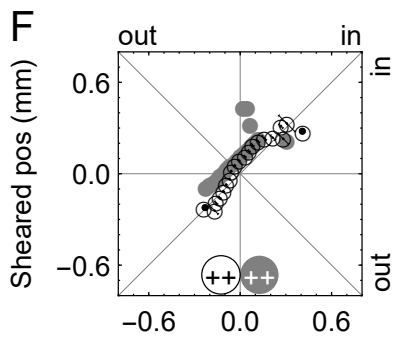

Relaxed pos 2NN (mm)

Figure S11: Summary of statistically significant Kendall $\tau$ correlations between absolute particle distribution positions, where $p<0.05$. “-” indicates a negative correlation where $-0.1<\tau<0$, "++" indicates a stronger positive correlation where $0.1<\tau<0.2$, and so on, where $-1<\tau<1$. Error bars indicate standard error with respect to the line $x=y$. 
Table S13: Summary of statistically significant Kendall $\tau$ correlations between relative particle distribution positions over the course of the print, where $p<0.05$. "." indicates a negative correlation where $-0.1<\tau<0$, " ++ " indicates a stronger positive correlation where $0.1<\tau<0.2$, and so on, where $-1<\tau<1$. Error bars indicate standard error with respect to the line $-x=y$ or $x=y$.

\section{Layer-by-layer}

Initial pos

Initial pos $\Delta$ pos relax NN $\Delta$ pos relax 2nd NN $\Delta$ pos shear

$\Delta$ pos relax NN

$\Delta$ pos relax 2nd NN

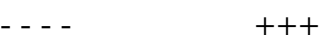

$\Delta$ pos shear

Bath

Initial pos

$\triangle$ pos relax NN

$\Delta$ pos relax 2nd NN

$++++--\cdot$

$\Delta$ pos shear

O Layer-by-layer Bath
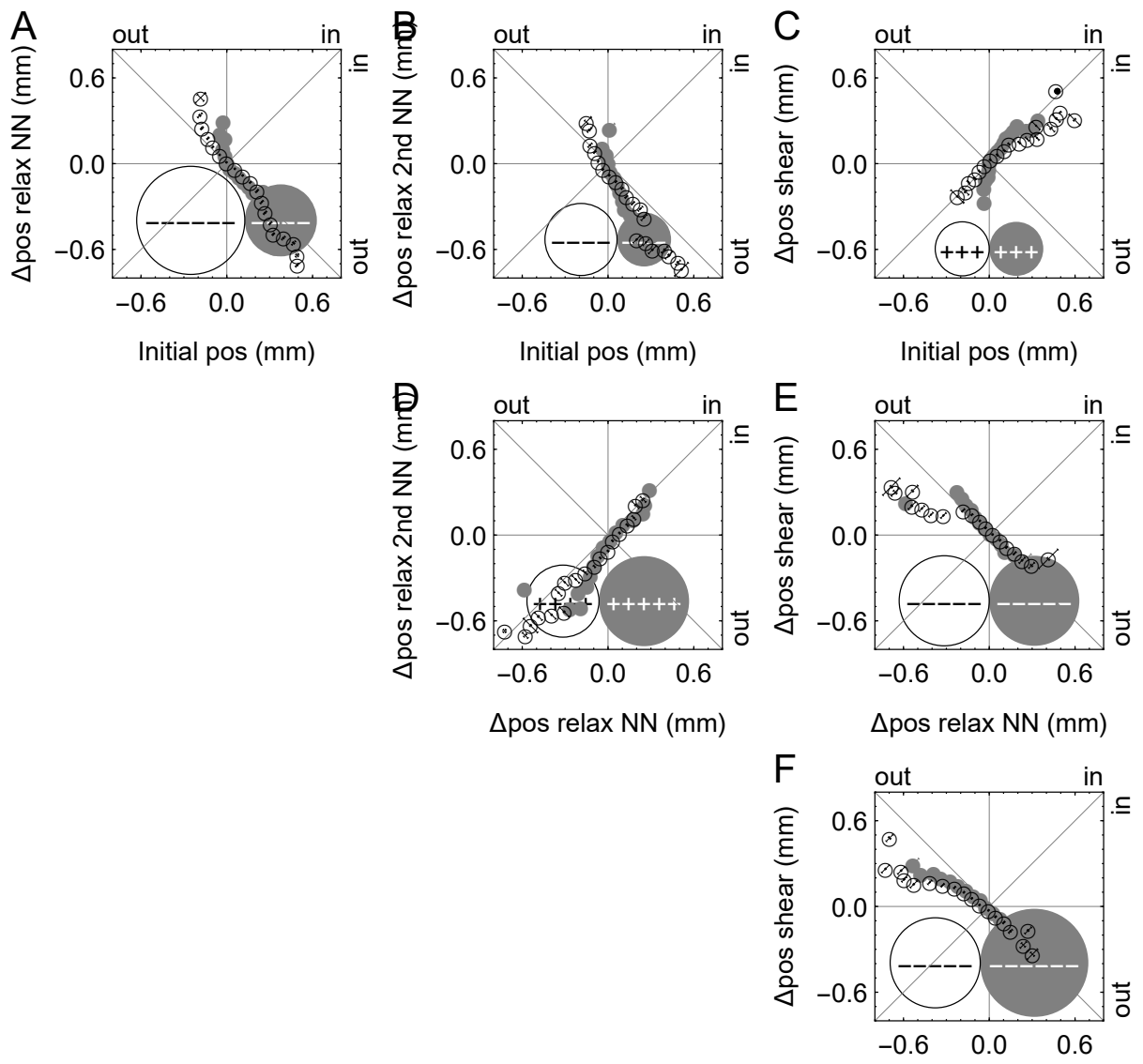

$\Delta$ pos relax $2 \mathrm{nd} \mathrm{NN}(\mathrm{mm})$

Figure S12: Summary of statistically significant Kendall $\tau$ correlations between relative particle distribution positions over the course of the print, where $p<0.05$. "-" indicates a negative correlation where $-0.1<\tau<0$, "++" indicates a stronger positive correlation where $0.1<\tau<0.2$, and so on, where $-1<\tau<1$. Error bars indicate standard error with respect to the line $x=y$. 
Table S14: Summary of statistically significant Kendall $\tau$ correlations between absolute particle distribution widths, where $p<0.05$. “-” indicates a negative correlation where $-0.1<\tau<0$, "++" indicates a stronger positive correlation where $0.1<\tau<0.2$, and so on, where $-1<\tau<1$.

Layer-by-layer

Initial width

Relaxed width NN

Initial width Relaxed width NN Relaxed width 2nd NN Sheared width

Relaxed width 2nd NN

$+++\quad++\quad+++$

Sheared width

Bath

Initial width

Relaxed width NN

Relaxed width 2nd NN

$++++$

Sheared width
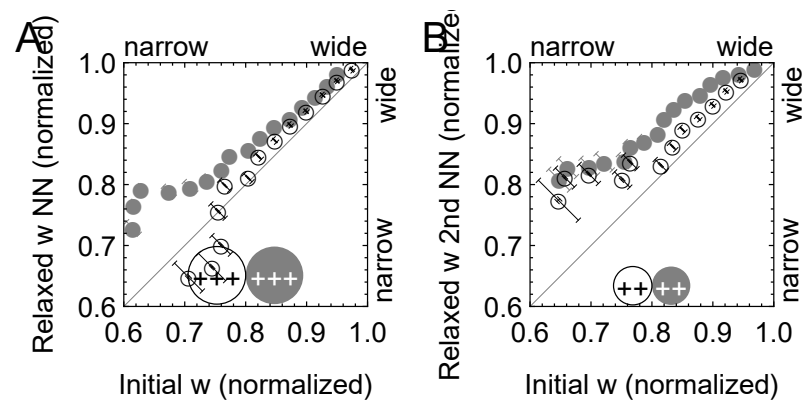

$\bigcirc$ Layer-by-layer $\bigcirc$ Bath
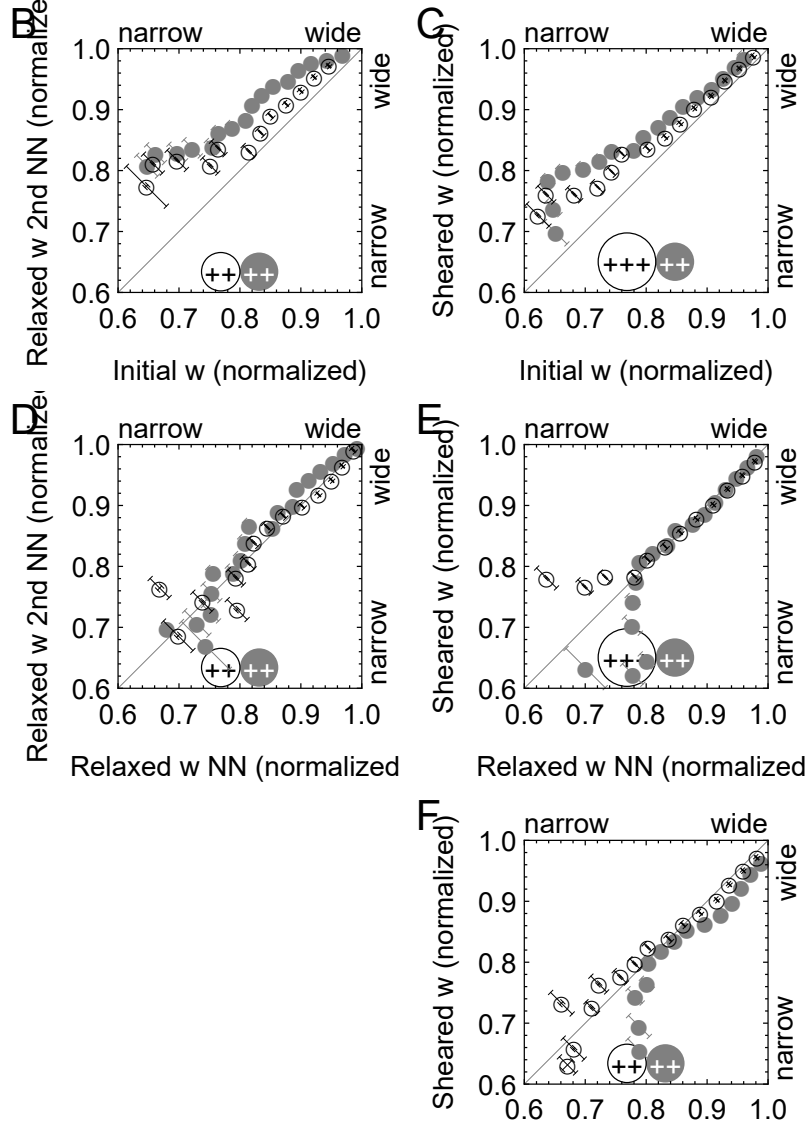

Relaxed w 2nd NN (normalizı

Figure S13: Summary of statistically significant Kendall $\tau$ correlations between absolute particle distribution widths, where $p<0.05$. “-” indicates a negative correlation where $-0.1<\tau<0$, “++" indicates a stronger positive correlation where $0.1<\tau<0.2$, and so on, where $-1<\tau<1$. Error bars indicate standard error with respect to the line $y=0$ or $-x=y$ or $x=y$. 
Table S15: Summary of statistically significant Kendall $\tau$ correlations between relative particle distribution widths over the course of the print, where $p<0.05$. "-" indicates a negative correlation where $-0.1<\tau<0$, "++" indicates a stronger positive correlation where $0.1<\tau<0.2$, and so on, where $-1<\tau<1$.

\author{
Layer-by-layer \\ Initial width \\ $\Delta$ width relax NN \\ $\Delta$ width relax 2nd NN \\ $\Delta$ width shear \\ Bath \\ Initial width \\ $\Delta$ width relax NN \\ $\Delta$ width relax 2nd NN \\ $\Delta$ width shear
}

\section{Initial width $\Delta$ width relax NN $\Delta$ width relax 2nd NN $\Delta$ width shear}
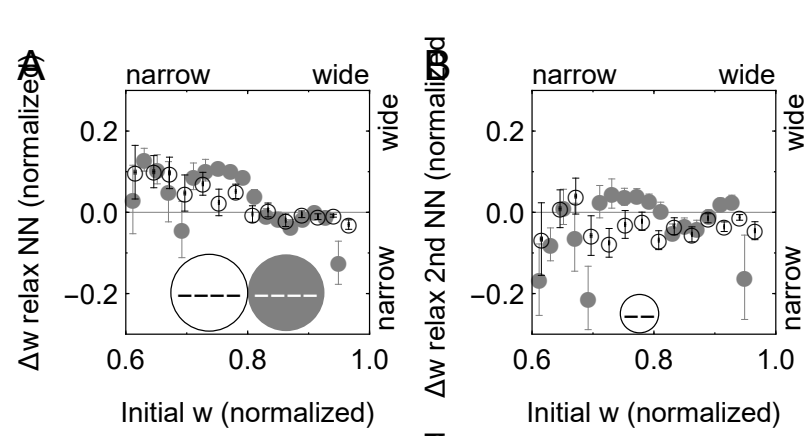

Layer-by-layer B Bath
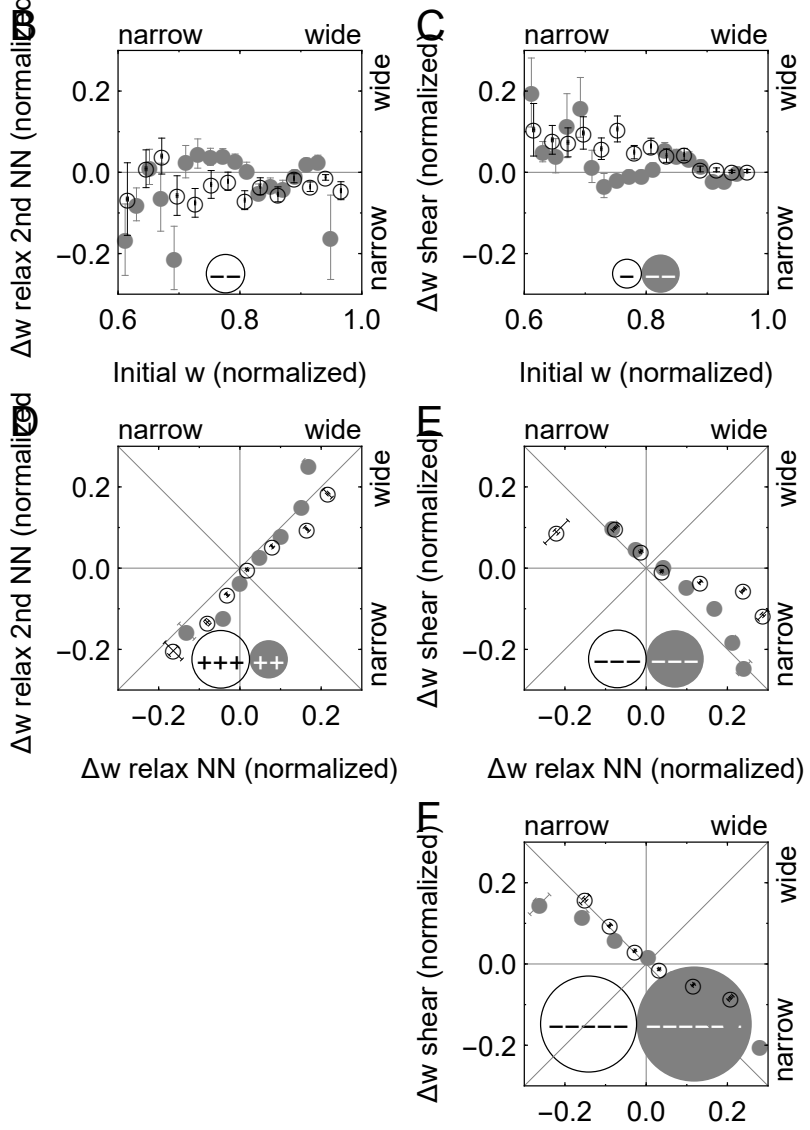

$\Delta \mathrm{w}$ relax $2 \mathrm{nd} \mathrm{NN}$ (normalize

Figure S14: Summary of statistically significant Kendall $\tau$ correlations between relative particle distribution widths over the course of the print, where $p<0.05$. "-" indicates a negative correlation where $-0.1<\tau<0$, "++" indicates a stronger positive correlation where $0.1<\tau<0.2$, and so on, where $-1<\tau<1$. Error bars indicate standard error with respect to the line $y=0$ or $-x=y$ or $x=y$. 
Table S16: Summary of statistically significant Kendall $\tau$ correlations between absolute particle distribution positions and widths, where $p<0.05$. "-" indicates a negative correlation where $-0.1<\tau<0$, "++" indicates a stronger positive correlation where $0.1<\tau<0.2$, and so on, where $-1<\tau<1$.

Layer-by-layer

Initial position Relaxed position NN Relaxed position 2nd NN Sheared position

Initial width

Relaxed width NN

Relaxed width 2nd NN

Sheared width

Bath

Initial width

Relaxed width NN

Relaxed width 2nd NN

Sheared width 

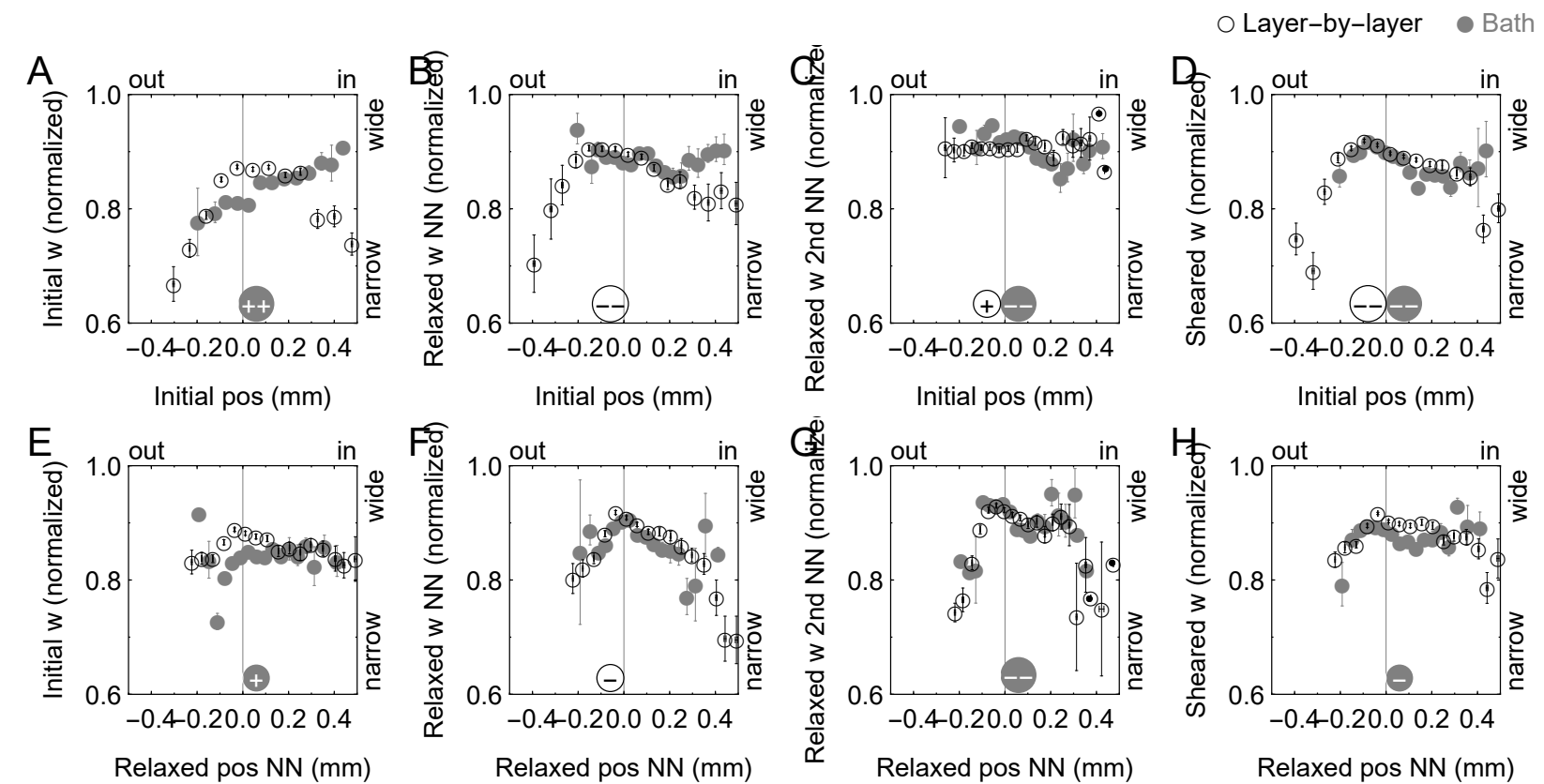

Relaxed pos NN (mm)

Relaxed pos NN (mm)
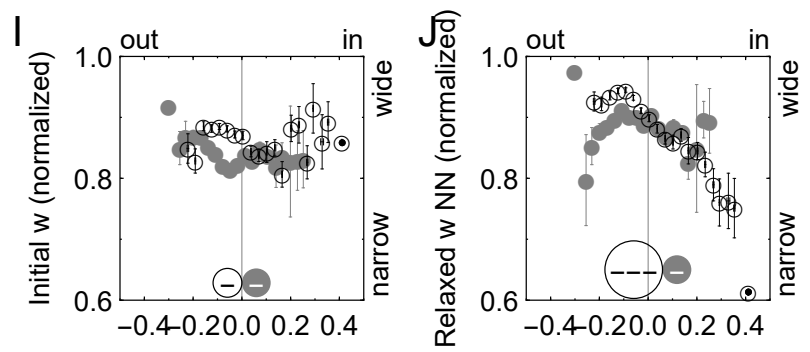

Relaxed pos 2NN (mm)

Relaxed pos 2NN (mm)
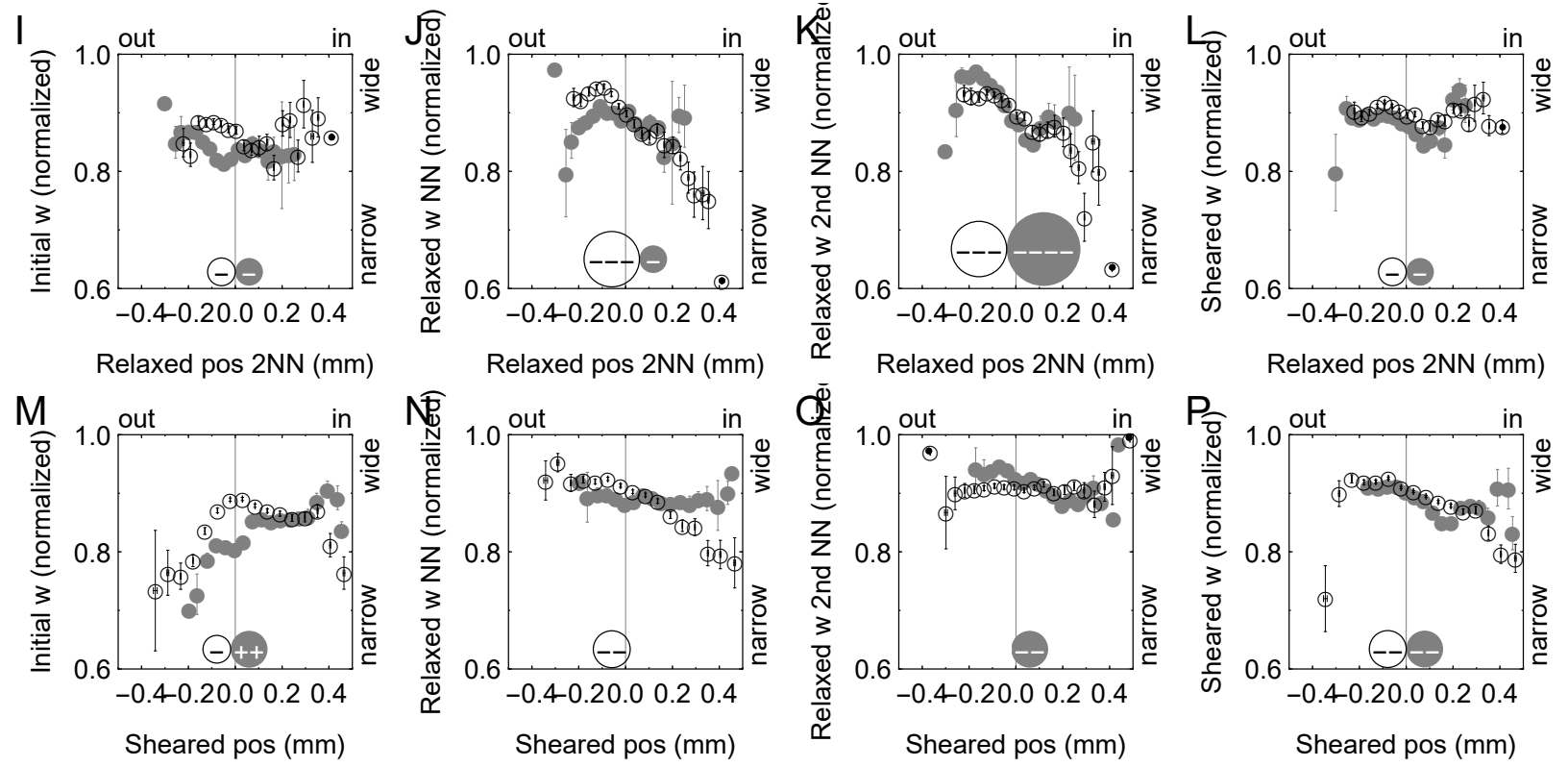

Relaxed pos 2NN (mm)

Relaxed pos 2NN (mm)
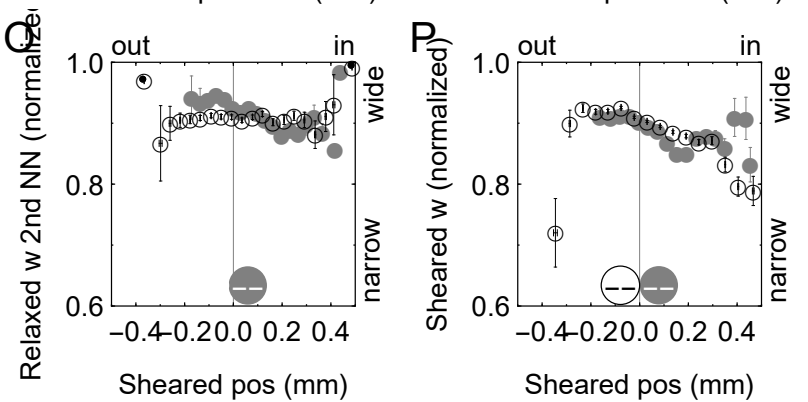

Figure S15: Summary of statistically significant Kendall $\tau$ correlations between absolute particle distribution positions and widths, where $p<0.05$. "-" indicates a negative correlation where $-0.1<\tau<0$, "++" indicates a stronger positive correlation where $0.1<\tau<0.2$, and so on, where $-1<\tau<1$. Error bars indicate standard error with respect to the line $y=0$. 
Table S17: Summary of statistically significant Kendall $\tau$ correlations between relative particle distribution positions and widths over the course of the print, where $p<0.05$. "-" indicates a negative correlation where $-0.1<\tau<0$, "++" indicates a stronger positive correlation where $0.1<\tau<0.2$, and so on, where $-1<\tau<1$.

\section{Layer-by-layer}

Initial width

$\Delta$ width relax NN

$\Delta$ width relax 2nd NN

$\Delta$ width shear

Bath

Initial width

$\Delta$ width relax NN

$\Delta$ width relax 2nd NN

$\Delta$ width shear

Initial pos $\quad \Delta$ pos relax NN $\Delta$ pos relax 2nd NN $\quad \Delta$ pos shear

$\begin{array}{cccc}-- & ++ & ++ & - \\ ++ & - & -- & + \\ - & & ++ & ++ \\ & & -- & + \\ ++ & & + & \\ -- & & -- & ++ \\ ++ & -- & ++ & -\end{array}$



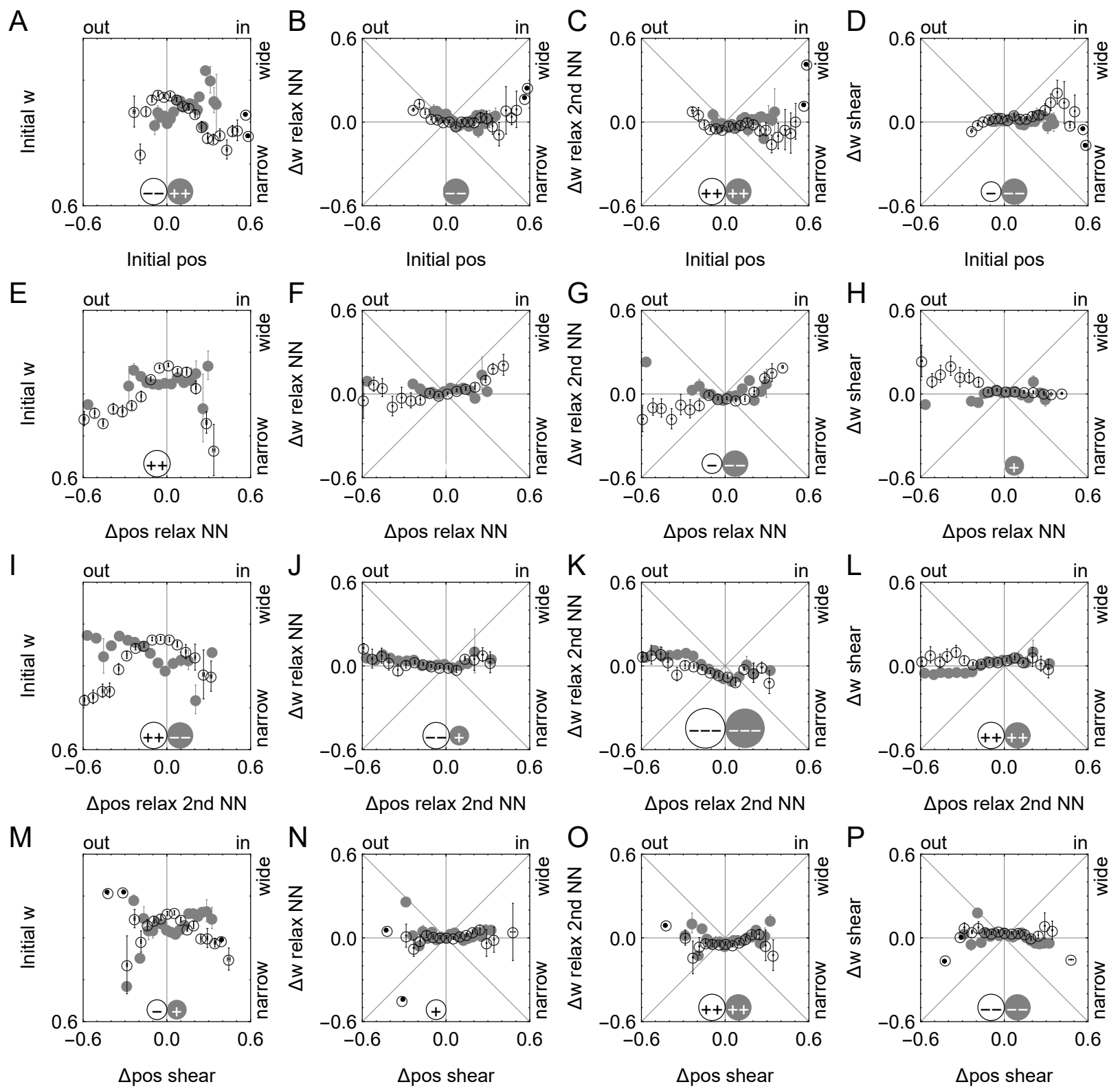

Figure S16: Summary of statistically significant Kendall $\tau$ correlations between relative particle distribution positions and widths over the course of the print, where $p<0.05$. "-" indicates a negative correlation where $-0.1<\tau<0$, " ++ " indicates a stronger positive correlation where $0.1<\tau<0.2$, and so on, where $-1<\tau<1$. Error bars indicate standard error with respect to the line $y=0$. 


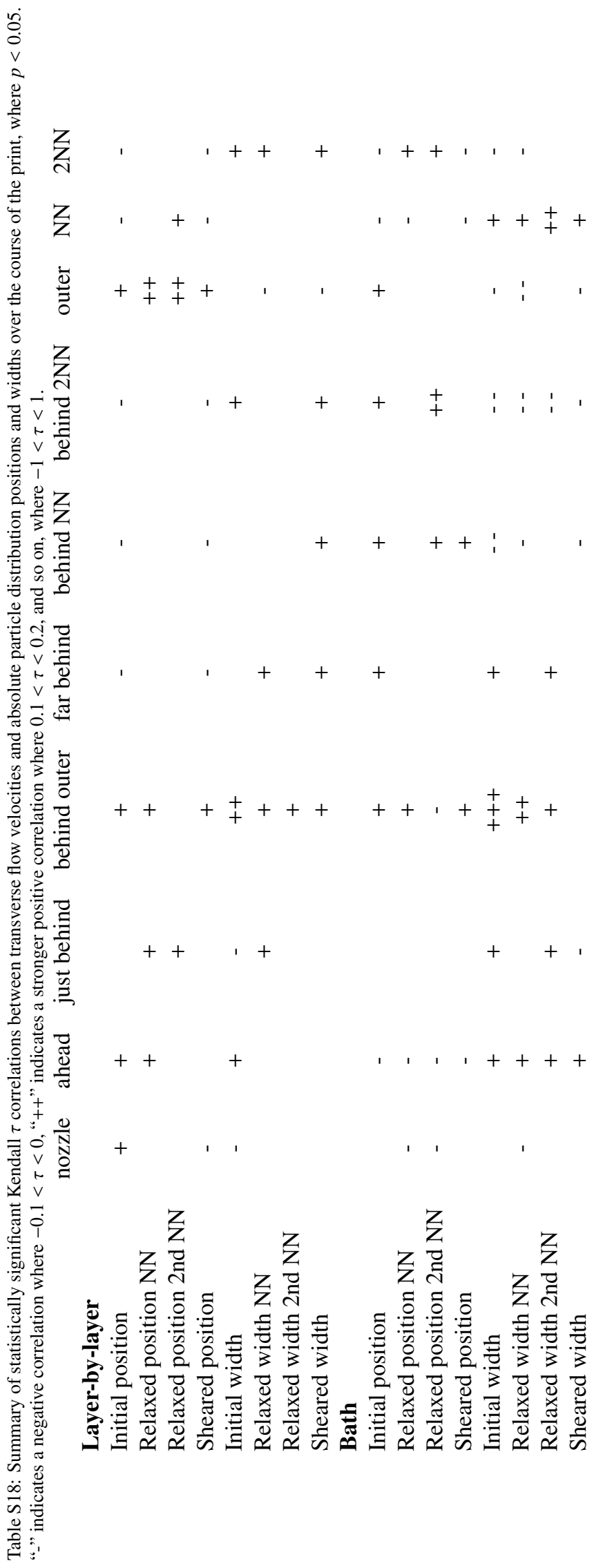




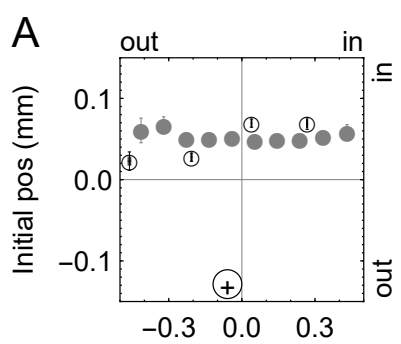

$v_{T}$ nozzle $(\mathrm{mm} / \mathrm{s})$

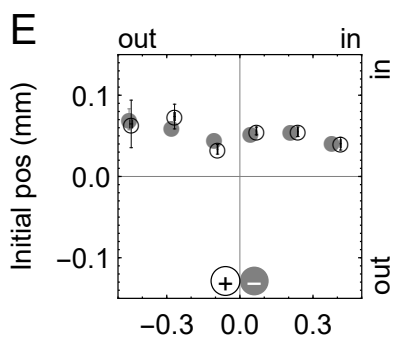

$v_{T}$ ahead $(\mathrm{mm} / \mathrm{s})$

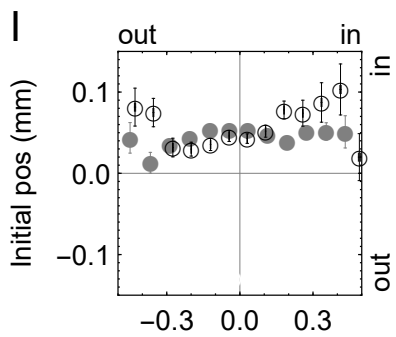

$v_{T}$ just behind $(\mathrm{mm} / \mathrm{s})$

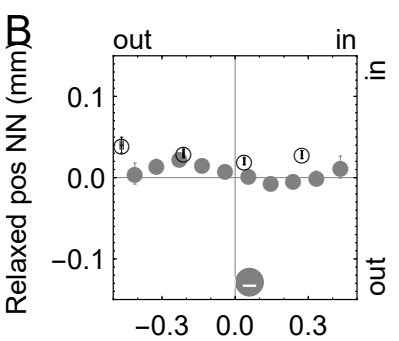

$v_{T}$ nozzle $(\mathrm{mm} / \mathrm{s})$

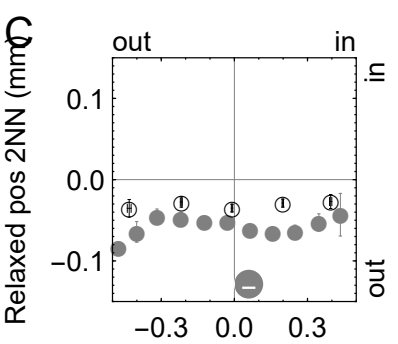

$v_{T}$ nozzle $(\mathrm{mm} / \mathrm{s})$

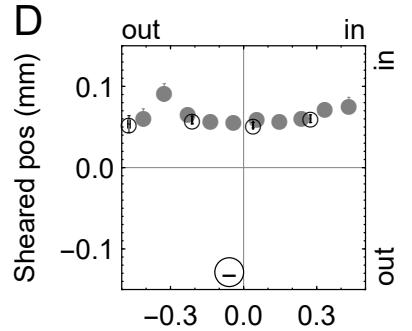

$v_{T}$ nozzle $(\mathrm{mm} / \mathrm{s})$

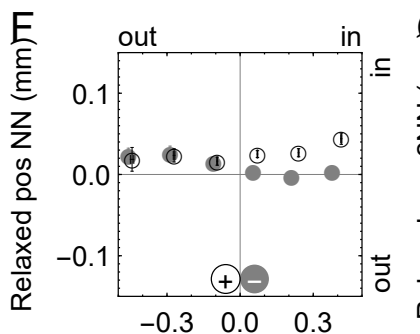

$v_{T}$ ahead $(\mathrm{mm} / \mathrm{s})$
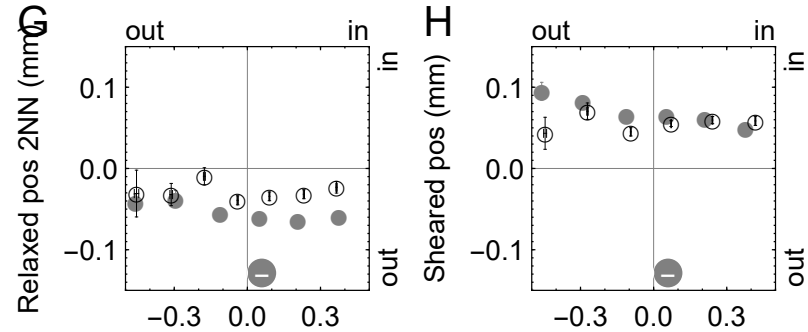

$v_{T}$ ahead $(\mathrm{mm} / \mathrm{s})$

$v_{T}$ ahead $(\mathrm{mm} / \mathrm{s})$

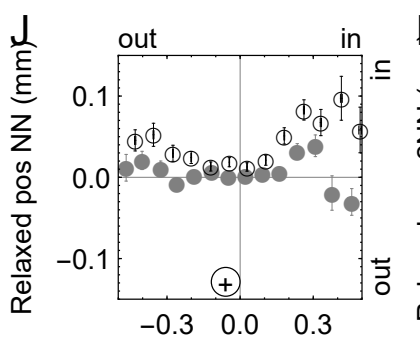

$v_{T}$ just behind $(\mathrm{mm} / \mathrm{s})$

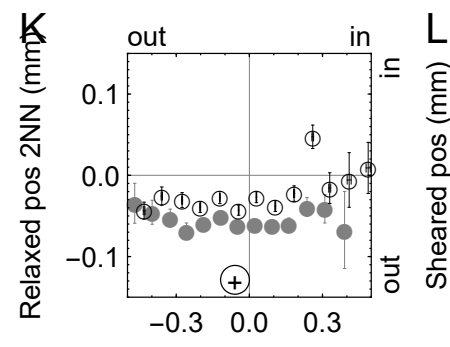

$v_{T}$ just behind $(\mathrm{mm} / \mathrm{s})$
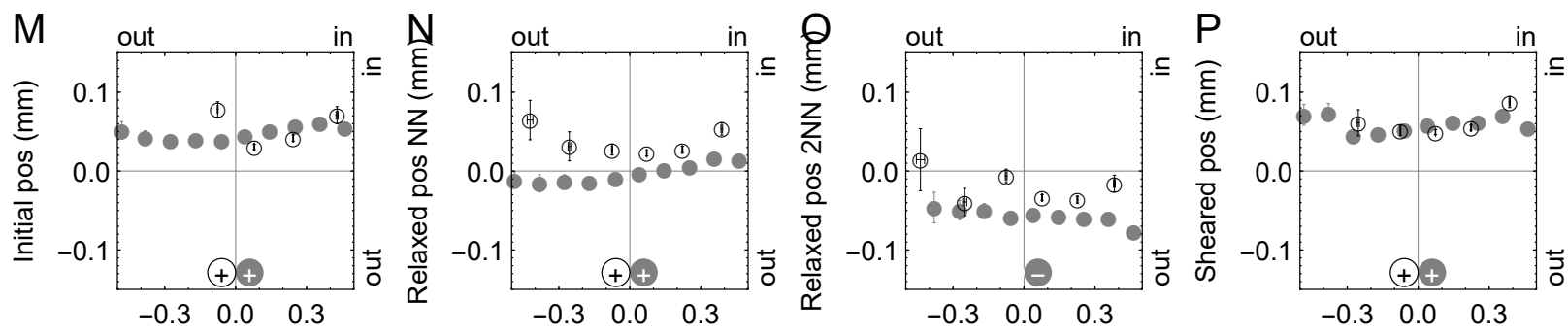

$v_{T}$ behind outer $(\mathrm{mm} / \mathrm{s})$

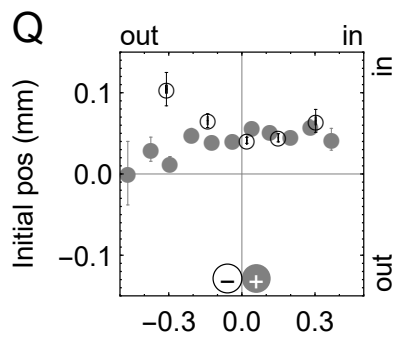

$v_{T}$ far behind $(\mathrm{mm} / \mathrm{s})$

$v_{T}$ behind outer $(\mathrm{mm} / \mathrm{s})$

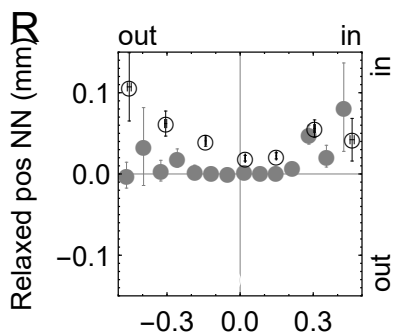

$v_{T}$ far behind $(\mathrm{mm} / \mathrm{s})$

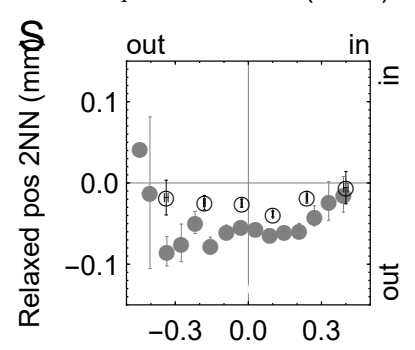

$v_{T}$ far behind $(\mathrm{mm} / \mathrm{s})$

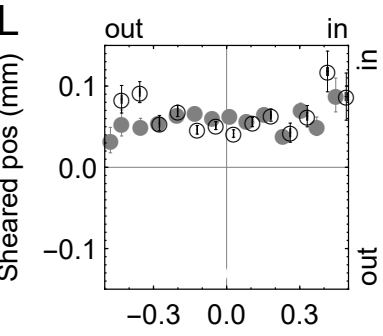

$v_{T}$ just behind $(\mathrm{mm} / \mathrm{s})$

$v_{T}$ behind outer $(\mathrm{mm} / \mathrm{s})$

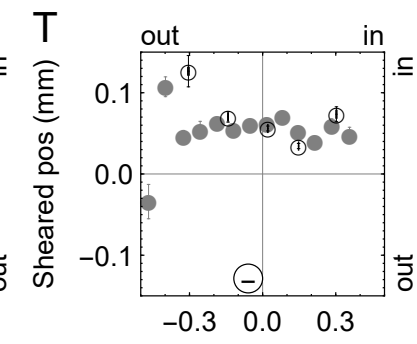

$v_{T}$ far behind $(\mathrm{mm} / \mathrm{s})$

Figure S17: Summary of statistically significant Kendall $\tau$ correlations between transverse flow velocities and absolute particle distribution positions and widths over the course of the print, where $p<0.05$. "-" indicates a negative correlation where $-0.1<\tau<0$, "++" indicates a stronger positive correlation where $0.1<\tau<0.2$, and so on, where $-1<\tau<1$. Error bars indicate standard error with respect to the line $y=0$. 


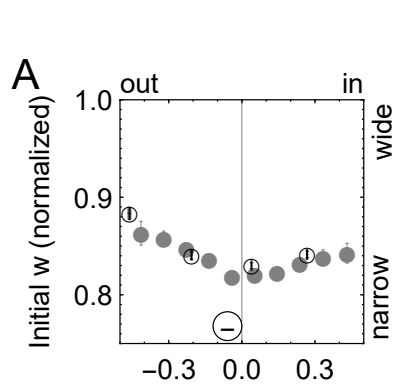

$v_{T}$ nozzle $(\mathrm{mm} / \mathrm{s})$

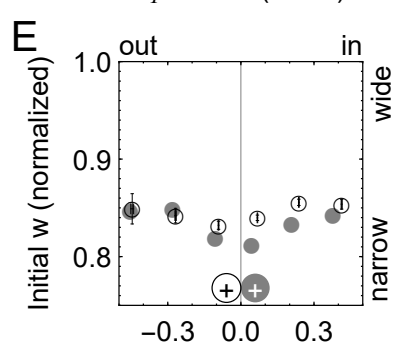

$v_{T}$ ahead $(\mathrm{mm} / \mathrm{s})$

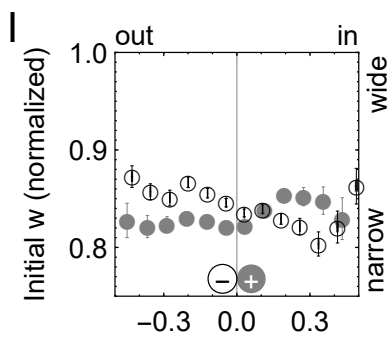

$v_{T}$ just behind $(\mathrm{mm} / \mathrm{s})$

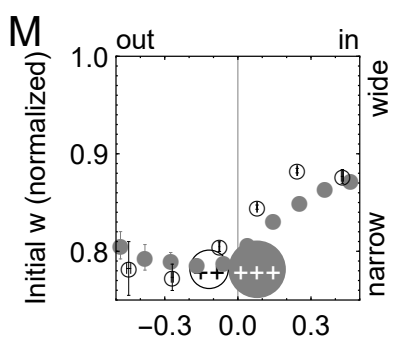

$v_{T}$ behind outer $(\mathrm{mm} / \mathrm{s})$

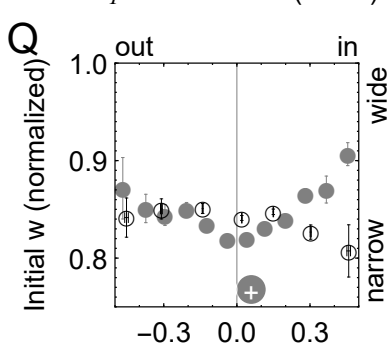

$v_{T}$ far behind $(\mathrm{mm} / \mathrm{s})$

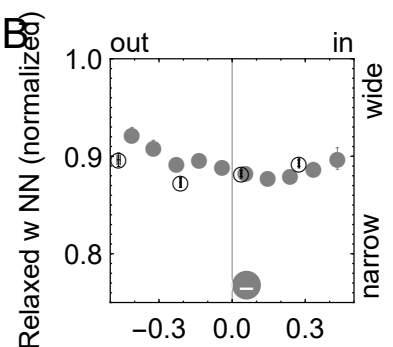

$v_{T}$ nozzle $(\mathrm{mm} / \mathrm{s})$

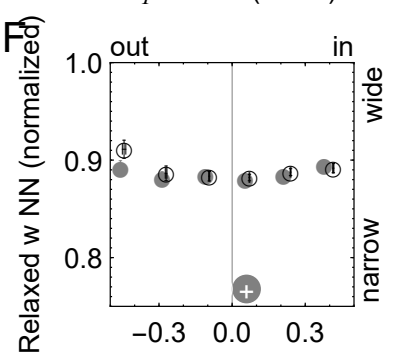

$v_{T}$ ahead (mm/s)

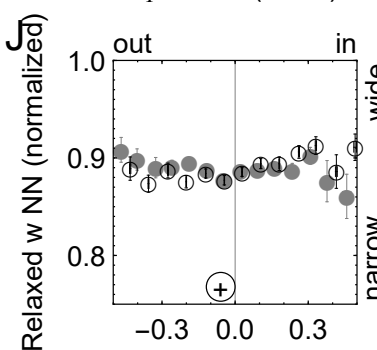

$v_{T}$ just behind $(\mathrm{mm} / \mathrm{s})$

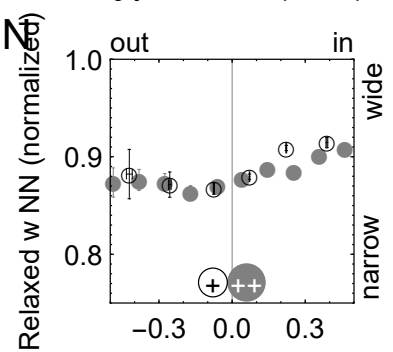

$v_{T}$ behind outer $(\mathrm{mm} / \mathrm{s})$

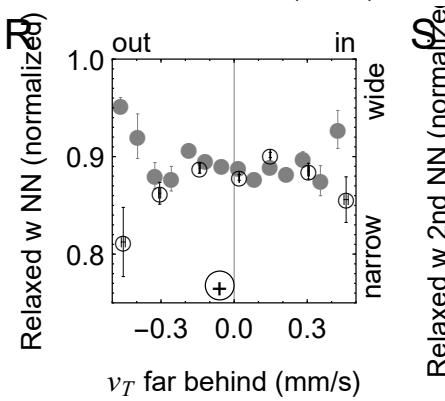

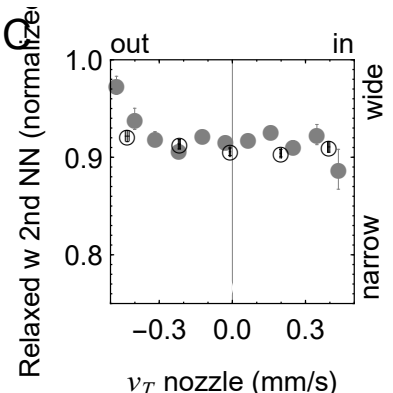

Layer-by-layer B Bath

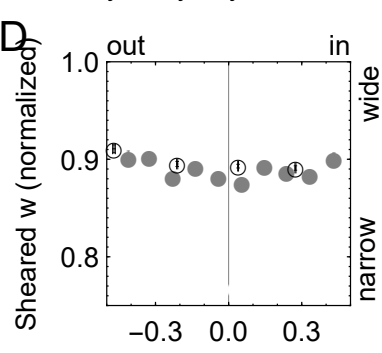

$v_{T}$ nozzle $(\mathrm{mm} / \mathrm{s})$
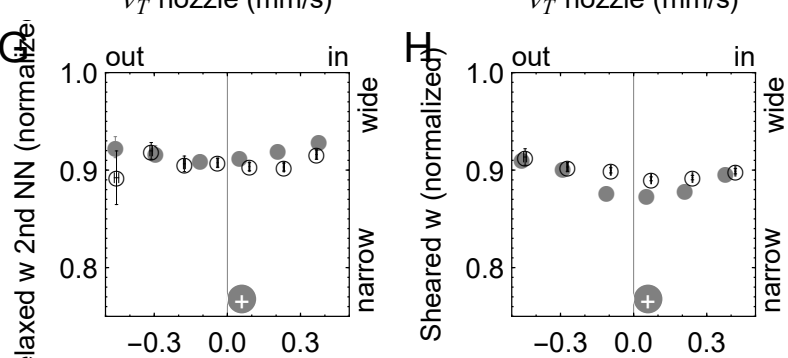

$v_{T}$ ahead $(\mathrm{mm} / \mathrm{s})$

$v_{T}$ ahead $(\mathrm{mm} / \mathrm{s})$

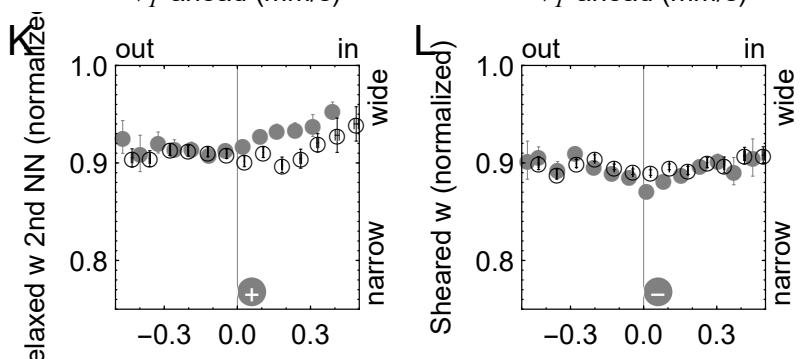

$v_{T}$ just behind (mm/s)

$v_{T}$ just behind $(\mathrm{mm} / \mathrm{s})$

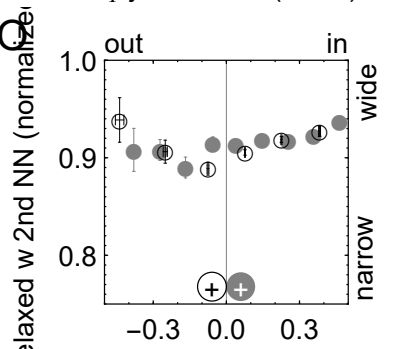

$v_{T}$ behind outer $(\mathrm{mm} / \mathrm{s})$

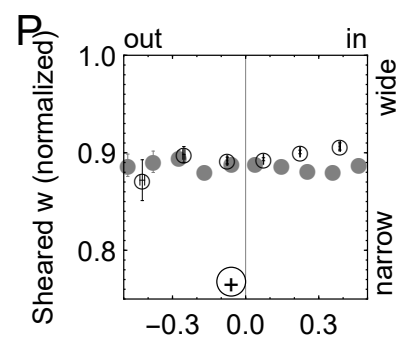

$v_{T}$ behind outer $(\mathrm{mm} / \mathrm{s})$

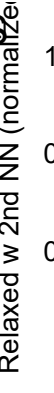

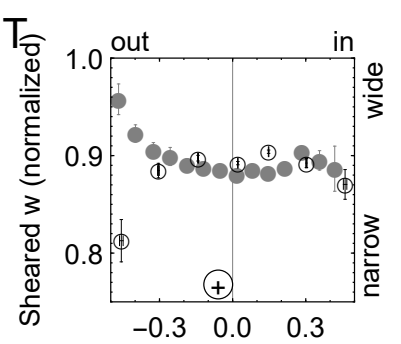

$v_{T}$ far behind $(\mathrm{mm} / \mathrm{s})$

Figure S18: Summary of statistically significant Kendall $\tau$ correlations between transverse flow velocities and absolute particle distribution positions and widths over the course of the print, where $p<0.05$. "-" indicates a negative correlation where $-0.1<\tau<0$, " ++ " indicates a stronger positive correlation where $0.1<\tau<0.2$, and so on, where $-1<\tau<1$. Error bars indicate standard error with respect to the line $y=0$. 


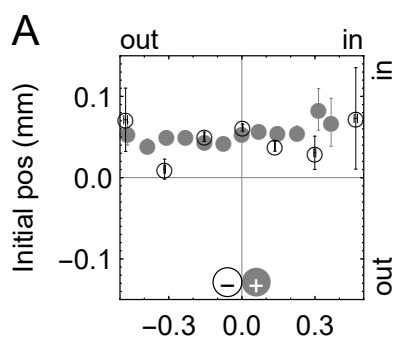

$v_{T}$ behind $\mathrm{NN}(\mathrm{mm} / \mathrm{s})$

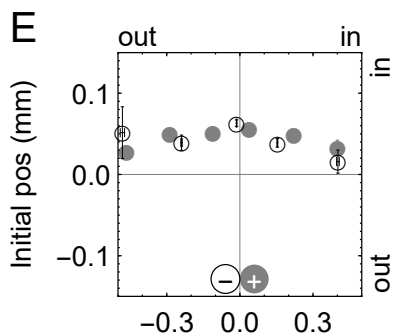

$v_{T}$ behind $2 \mathrm{NN}(\mathrm{mm} / \mathrm{s})$

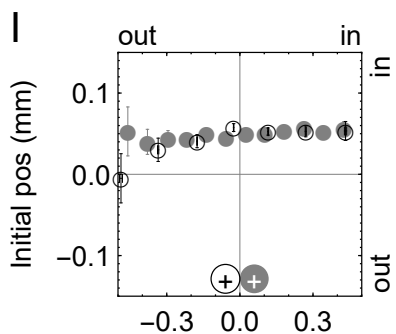

$v_{T}$ outer $(\mathrm{mm} / \mathrm{s})$

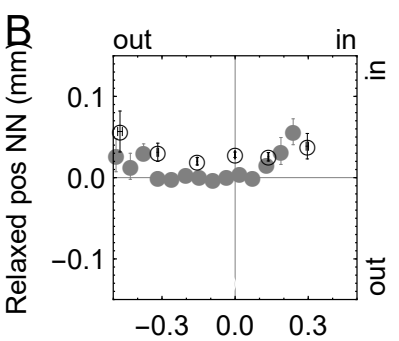

$v_{T}$ behind $\mathrm{NN}(\mathrm{mm} / \mathrm{s})$

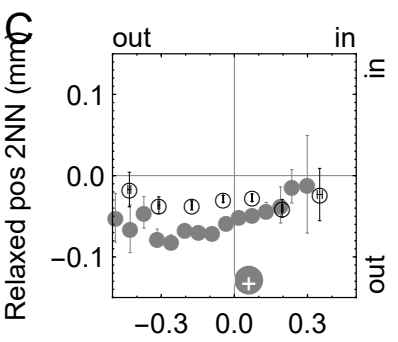

$v_{T}$ behind $\mathrm{NN}(\mathrm{mm} / \mathrm{s})$
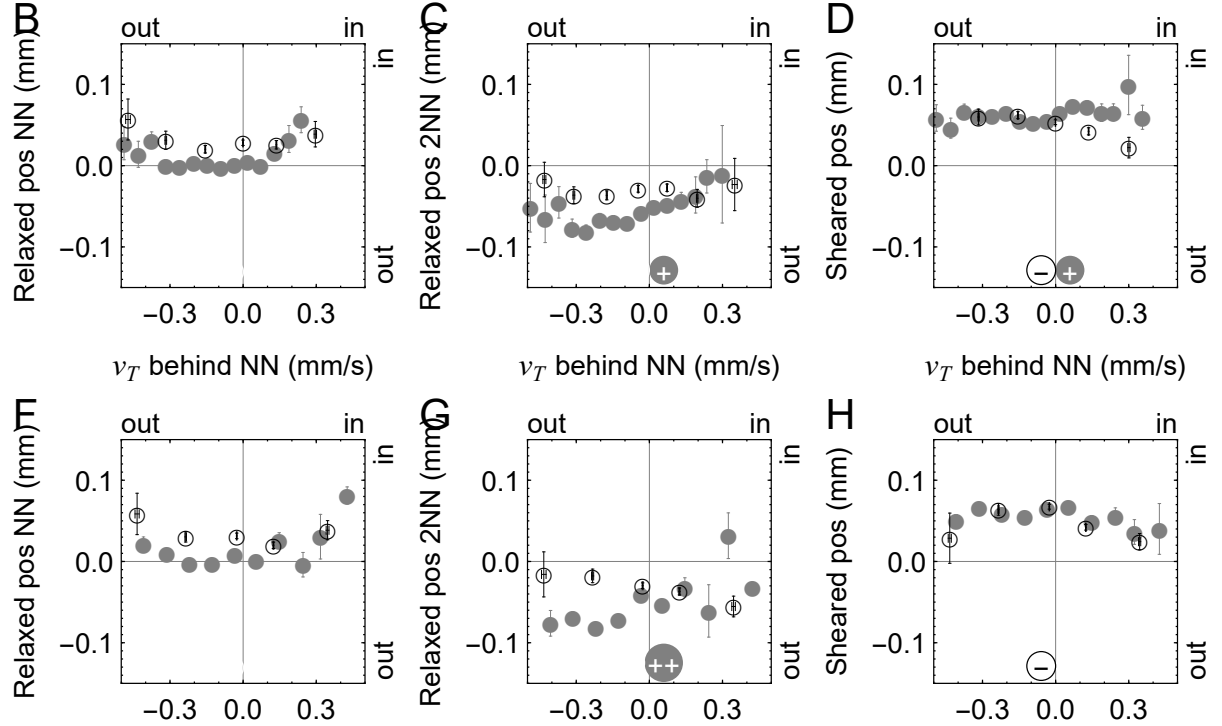

$v_{T}$ behind $2 \mathrm{NN}(\mathrm{mm} / \mathrm{s})$

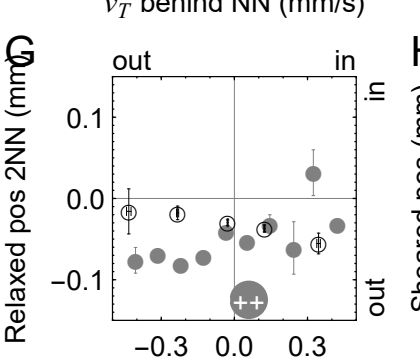

$v_{T}$ behind $\mathrm{NN}(\mathrm{mm} / \mathrm{s})$

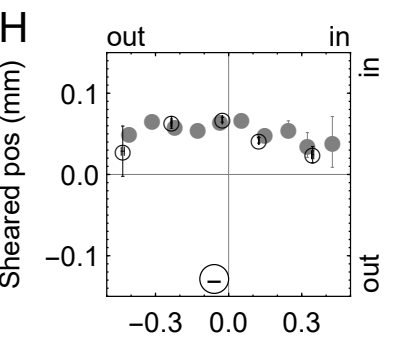

$v_{T}$ behind $2 \mathrm{NN}(\mathrm{mm} / \mathrm{s})$

$v_{T}$ behind $2 \mathrm{NN}(\mathrm{mm} / \mathrm{s})$
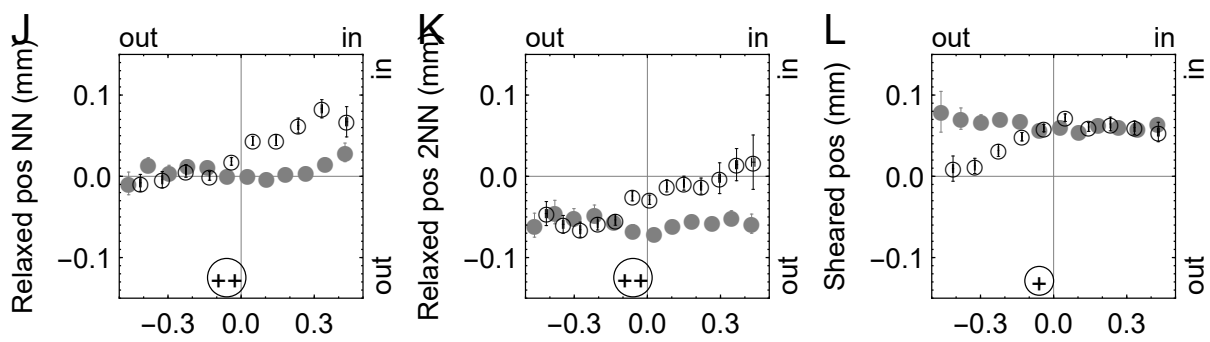

$v_{T}$ outer $(\mathrm{mm} / \mathrm{s})$

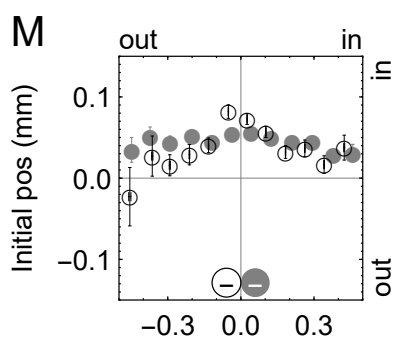

$v_{T}$ outer $(\mathrm{mm} / \mathrm{s})$
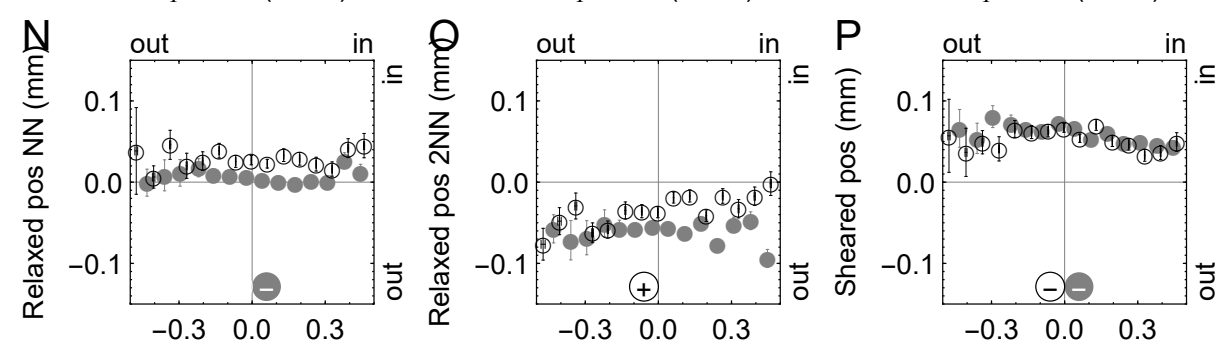

$v_{T} \mathrm{NN}(\mathrm{mm} / \mathrm{s})$
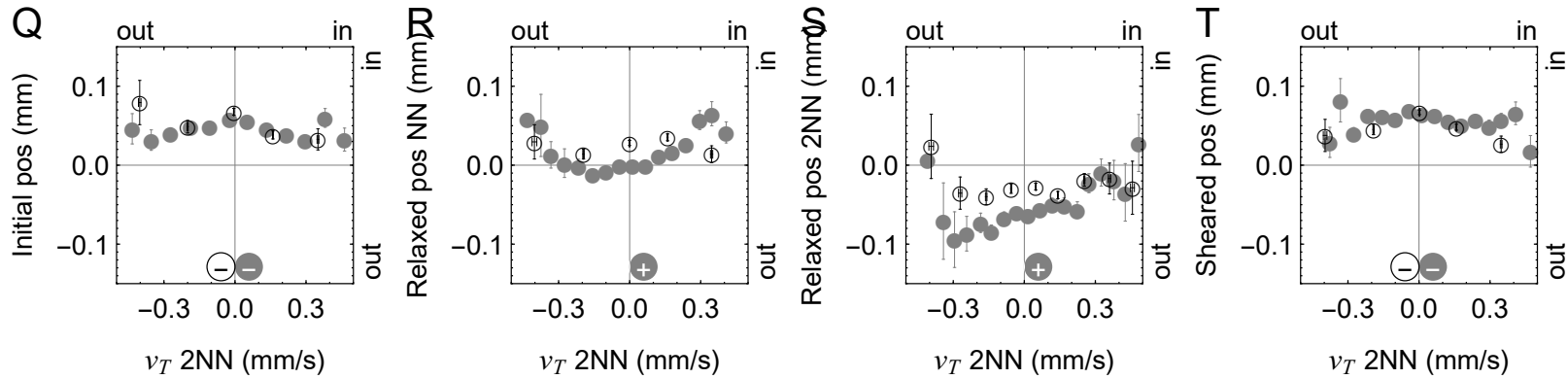

Figure S19: Summary of statistically significant Kendall $\tau$ correlations between transverse flow velocities and absolute particle distribution positions and widths over the course of the print, where $p<0.05$. "-" indicates a negative correlation where $-0.1<\tau<0$, " ++ " indicates a stronger positive correlation where $0.1<\tau<0.2$, and so on, where $-1<\tau<1$. Error bars indicate standard error with respect to the line $y=0$. 


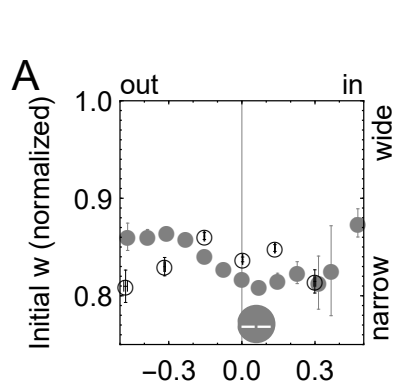

$v_{T}$ behind $\mathrm{NN}(\mathrm{mm} / \mathrm{s})$

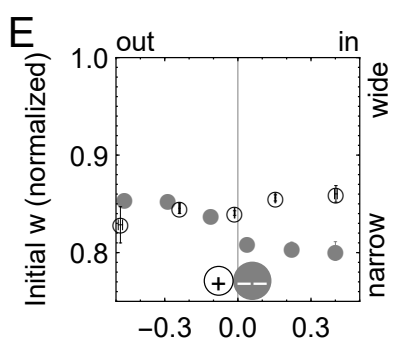

$v_{T}$ behind $2 \mathrm{NN}(\mathrm{mm} / \mathrm{s})$

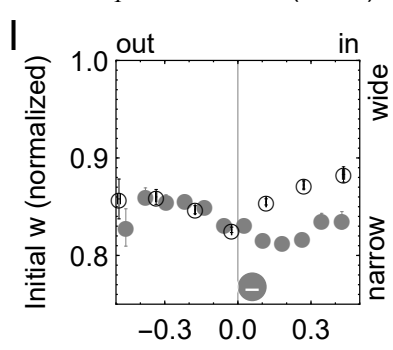

$v_{T}$ outer $(\mathrm{mm} / \mathrm{s})$

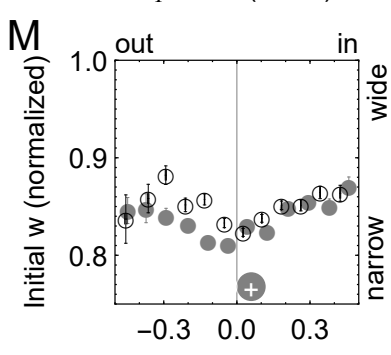

$v_{T} \mathrm{NN}(\mathrm{mm} / \mathrm{s})$

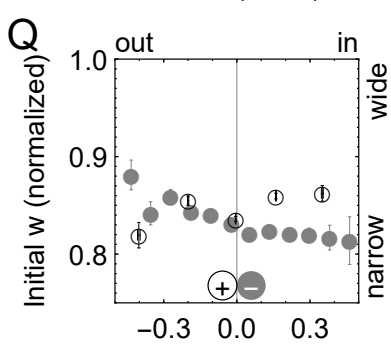

$v_{T} 2 \mathrm{NN}(\mathrm{mm} / \mathrm{s})$

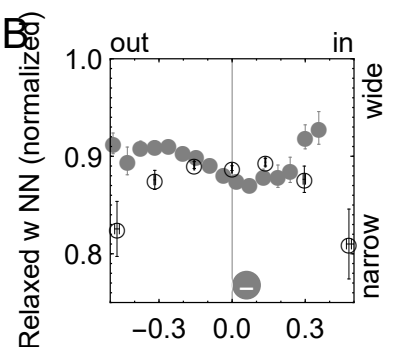

$v_{T}$ behind $\mathrm{NN}(\mathrm{mm} / \mathrm{s})$

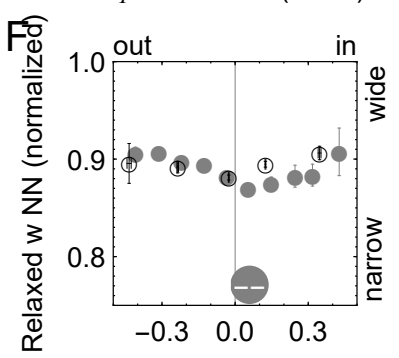

$v_{T}$ behind $2 \mathrm{NN}(\mathrm{mm} / \mathrm{s})$

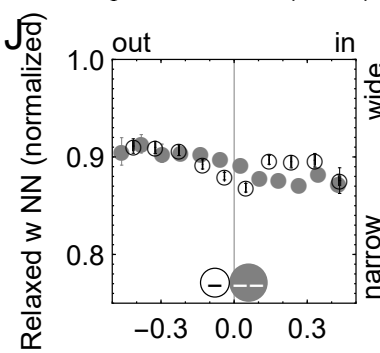

$v_{T}$ outer $(\mathrm{mm} / \mathrm{s})$

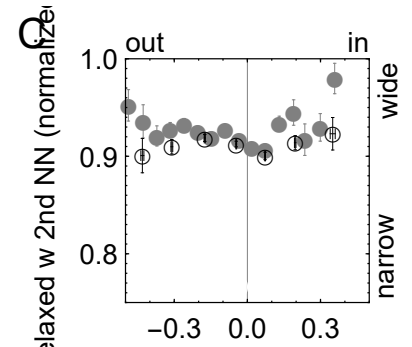

$v_{T}$ behind $\mathrm{NN}(\mathrm{mm} / \mathrm{s})$
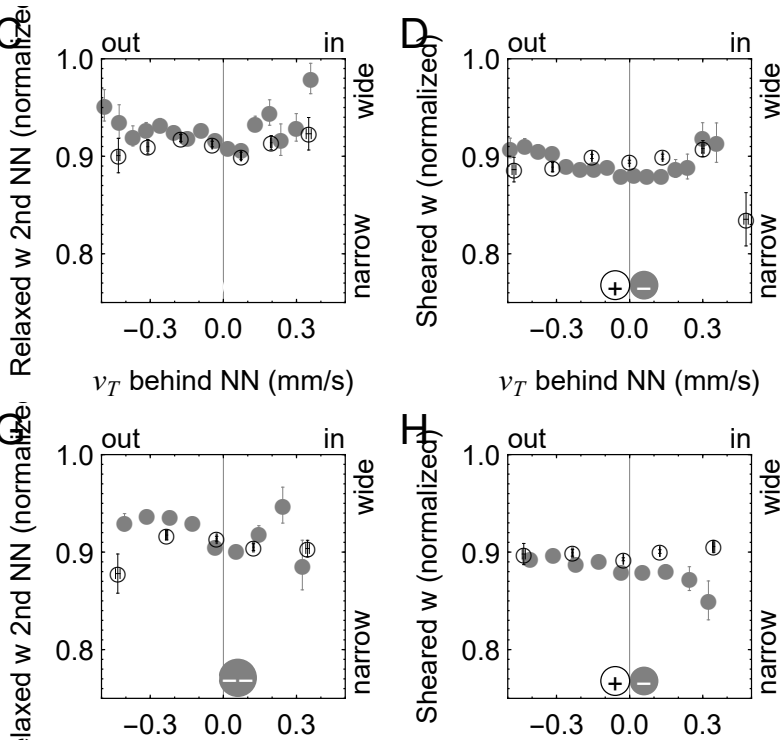

$v_{T}$ behind $\mathrm{NN}(\mathrm{mm} / \mathrm{s})$

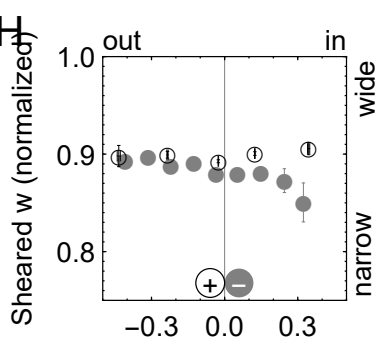

$v_{T}$ behind $2 \mathrm{NN}(\mathrm{mm} / \mathrm{s})$

$v_{T}$ behind $2 \mathrm{NN}(\mathrm{mm} / \mathrm{s})$

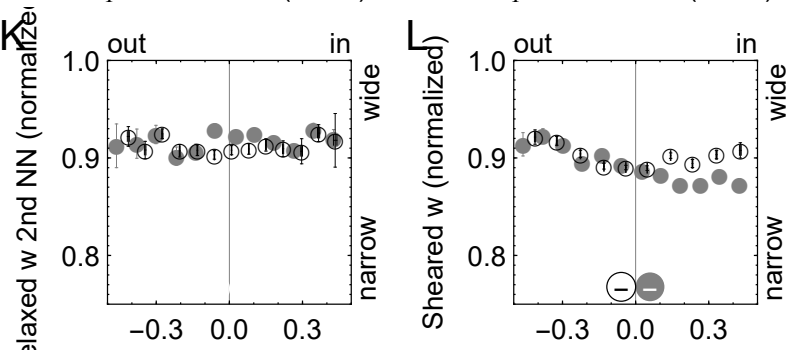

$v_{T}$ outer $(\mathrm{mm} / \mathrm{s})$

$v_{T}$ outer $(\mathrm{mm} / \mathrm{s})$
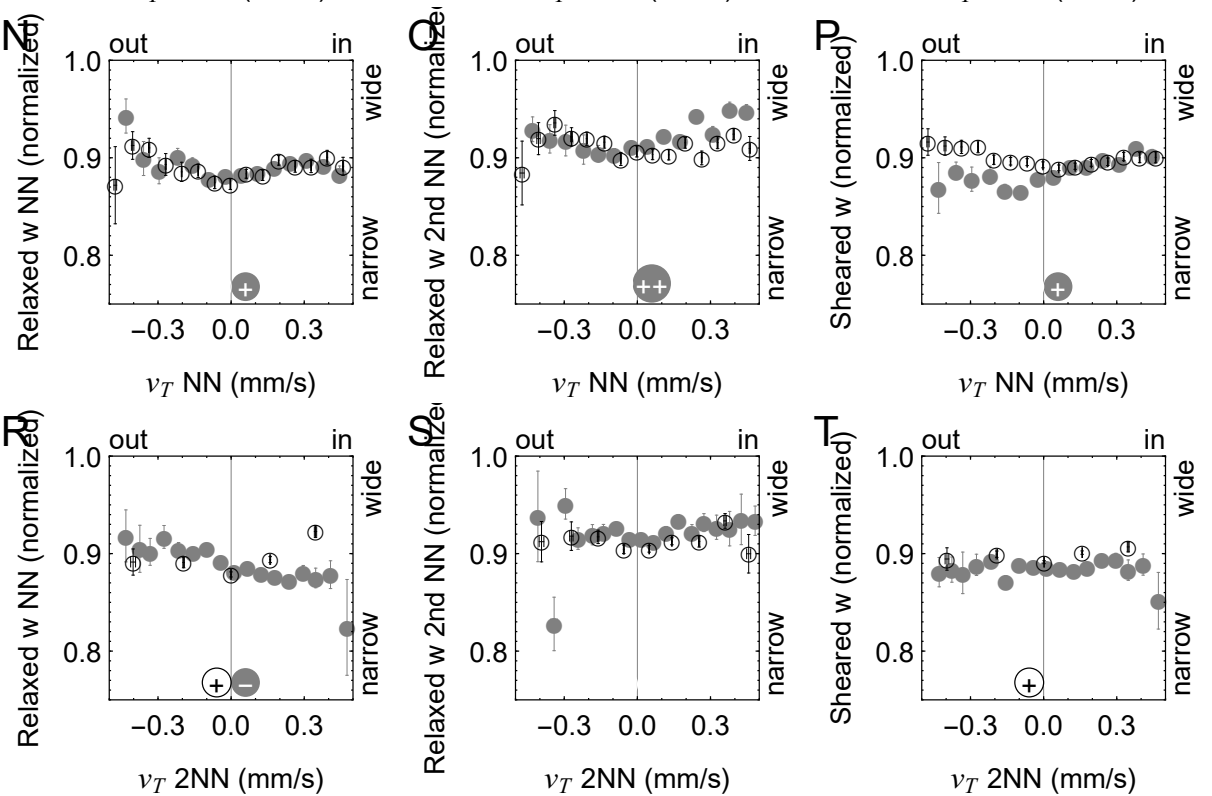

Figure S20: Summary of statistically significant Kendall $\tau$ correlations between transverse flow velocities and absolute particle distribution positions and widths over the course of the print, where $p<0.05$. "-" indicates a negative correlation where $-0.1<\tau<0$, " ++ " indicates a stronger positive correlation where $0.1<\tau<0.2$, and so on, where $-1<\tau<1$. Error bars indicate standard error with respect to the line $y=0$. 


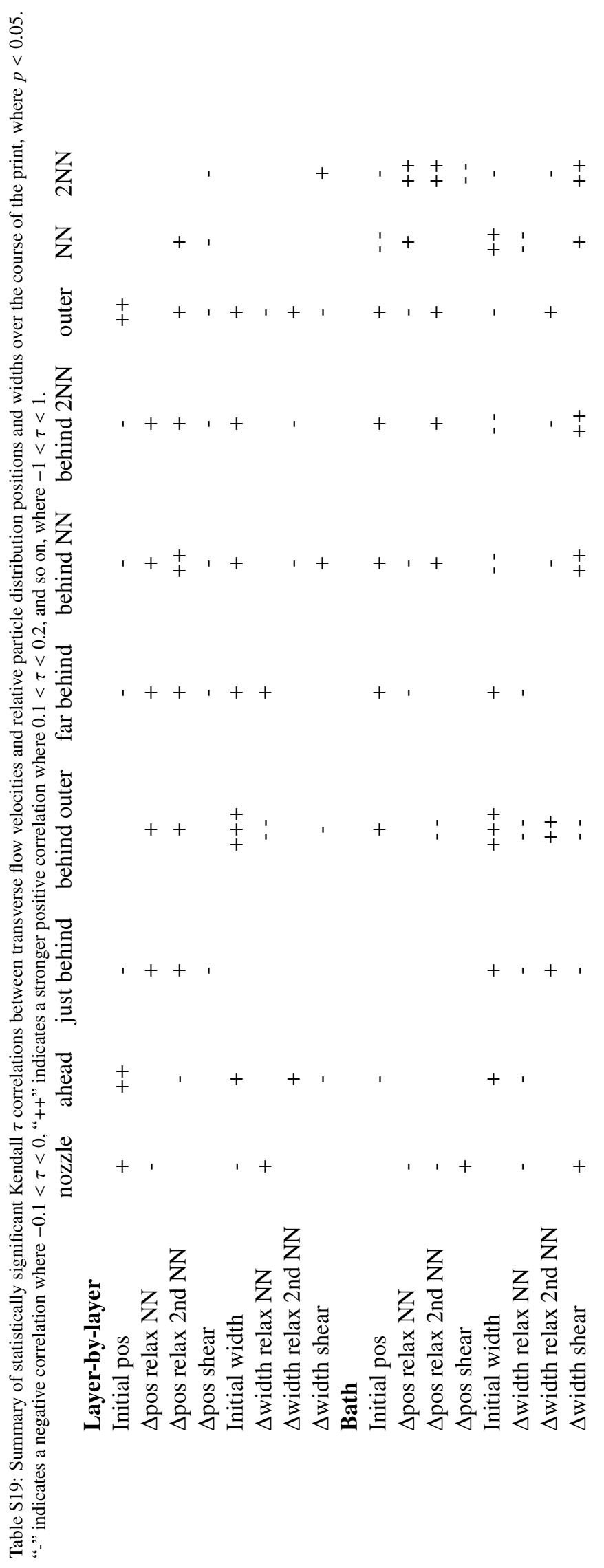




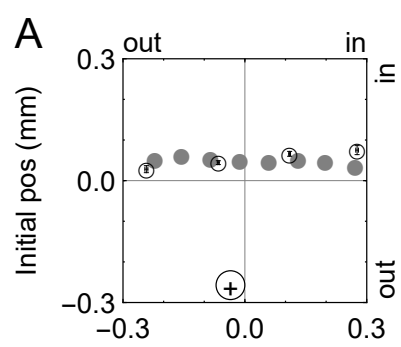

$v_{T}$ nozzle $(\mathrm{mm} / \mathrm{s})$

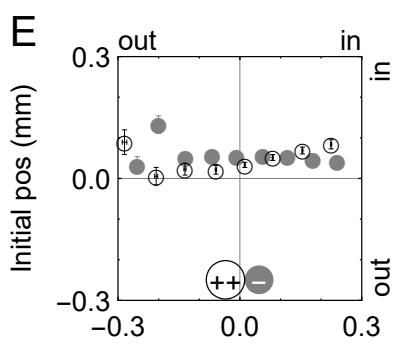

$v_{T}$ ahead $(\mathrm{mm} / \mathrm{s})$

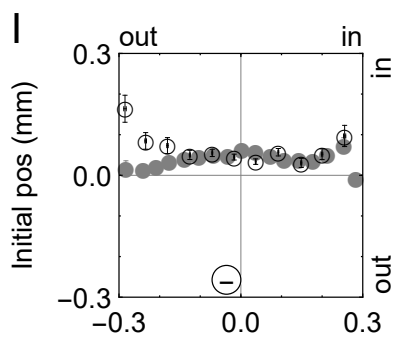

$v_{T}$ just behind $(\mathrm{mm} / \mathrm{s})$

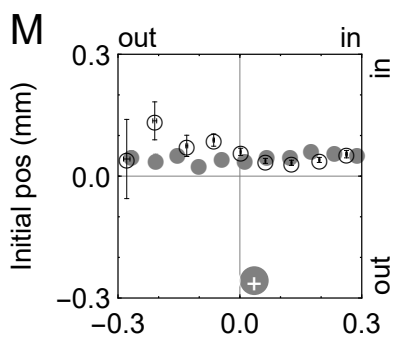

$v_{T}$ behind outer $(\mathrm{mm} / \mathrm{s})$

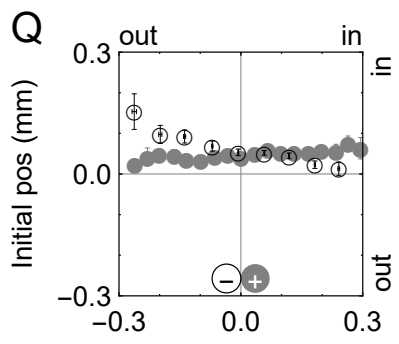

$v_{T}$ far behind $(\mathrm{mm} / \mathrm{s})$

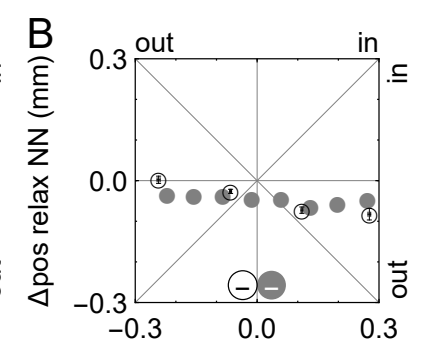

$v_{T}$ nozzle $(\mathrm{mm} / \mathrm{s})$

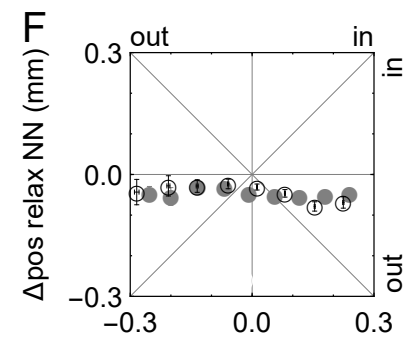

$v_{T}$ ahead $(\mathrm{mm} / \mathrm{s})$

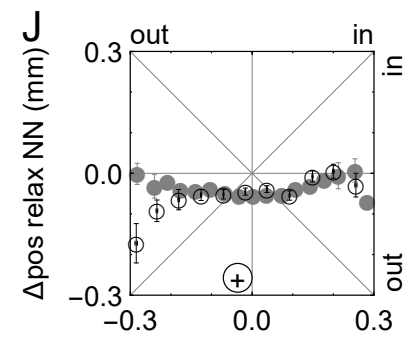

$v_{T}$ just behind $(\mathrm{mm} / \mathrm{s})$

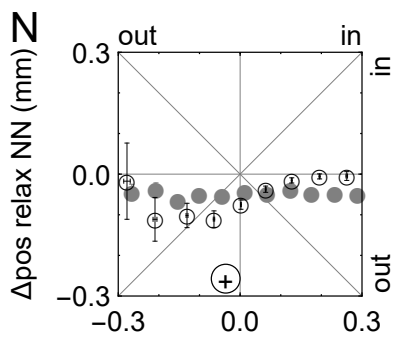

$v_{T}$ behind outer $(\mathrm{mm} / \mathrm{s})$

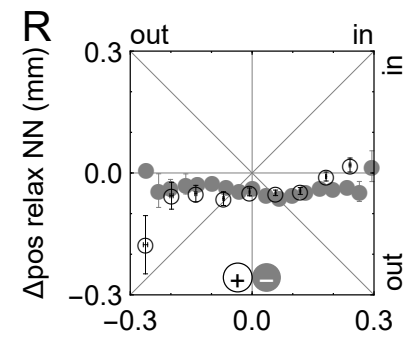

$v_{T}$ far behind $(\mathrm{mm} / \mathrm{s})$

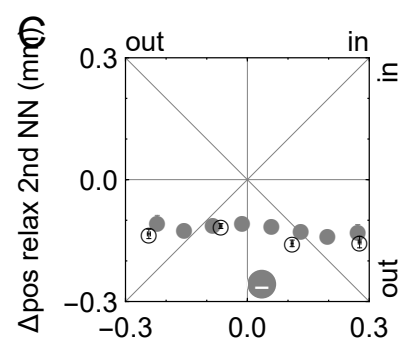

$v_{T}$ nozzle $(\mathrm{mm} / \mathrm{s})$

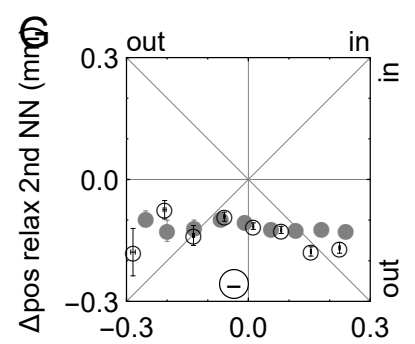

$v_{T}$ ahead $(\mathrm{mm} / \mathrm{s})$
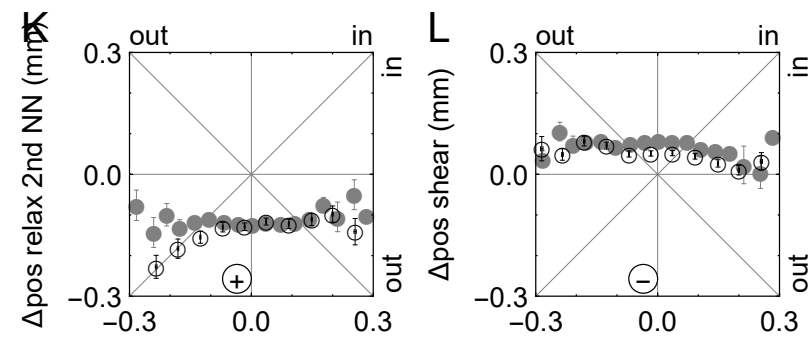

$v_{T}$ just behind $(\mathrm{mm} / \mathrm{s})$

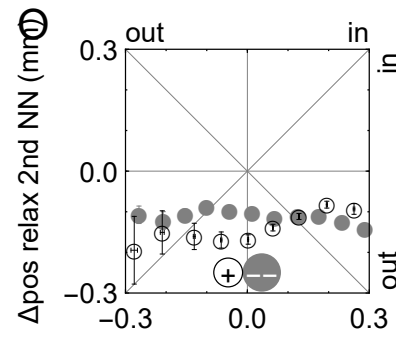

$v_{T}$ behind outer $(\mathrm{mm} / \mathrm{s})$

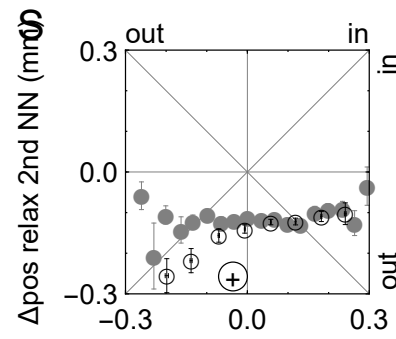

$v_{T}$ far behind $(\mathrm{mm} / \mathrm{s})$
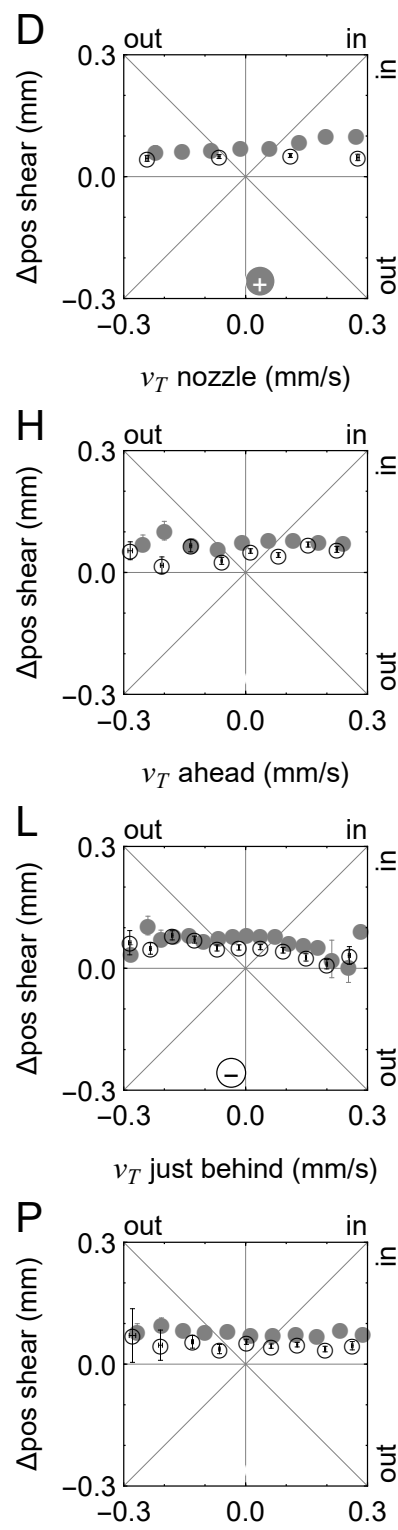

$v_{T}$ nozzle $(\mathrm{mm} / \mathrm{s})$

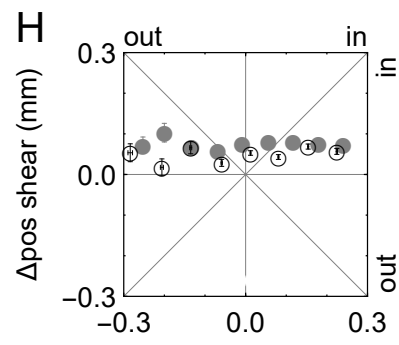

$v_{T}$ ahead $(\mathrm{mm} / \mathrm{s})$

$v_{T}$ behind outer $(\mathrm{mm} / \mathrm{s})$

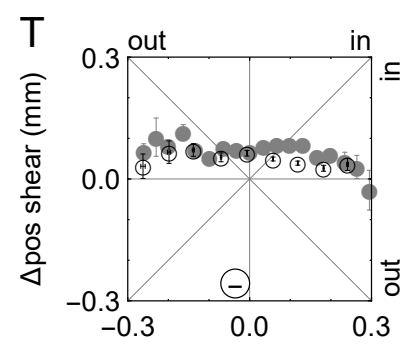

$v_{T}$ far behind $(\mathrm{mm} / \mathrm{s})$

Figure S21: Summary of statistically significant Kendall $\tau$ correlations between transverse flow velocities and relative particle distribution positions and widths over the course of the print, where $p<0.05$. "-" indicates a negative correlation where $-0.1<\tau<0$, "++" indicates a stronger positive correlation where $0.1<\tau<0.2$, and so on, where $-1<\tau<1$. Error bars indicate standard error with respect to the line $y=0$. 

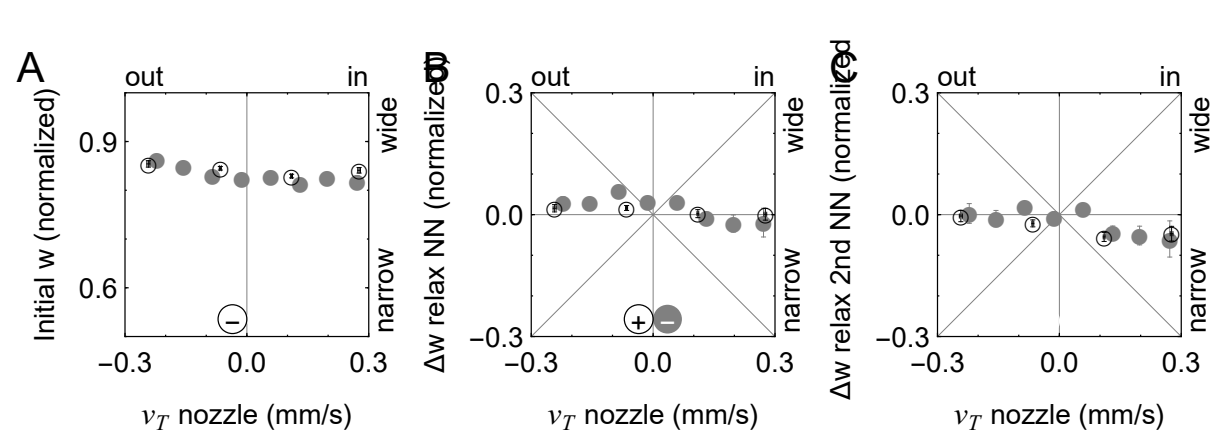

$\bigcirc$ Layer-by-layer Bath
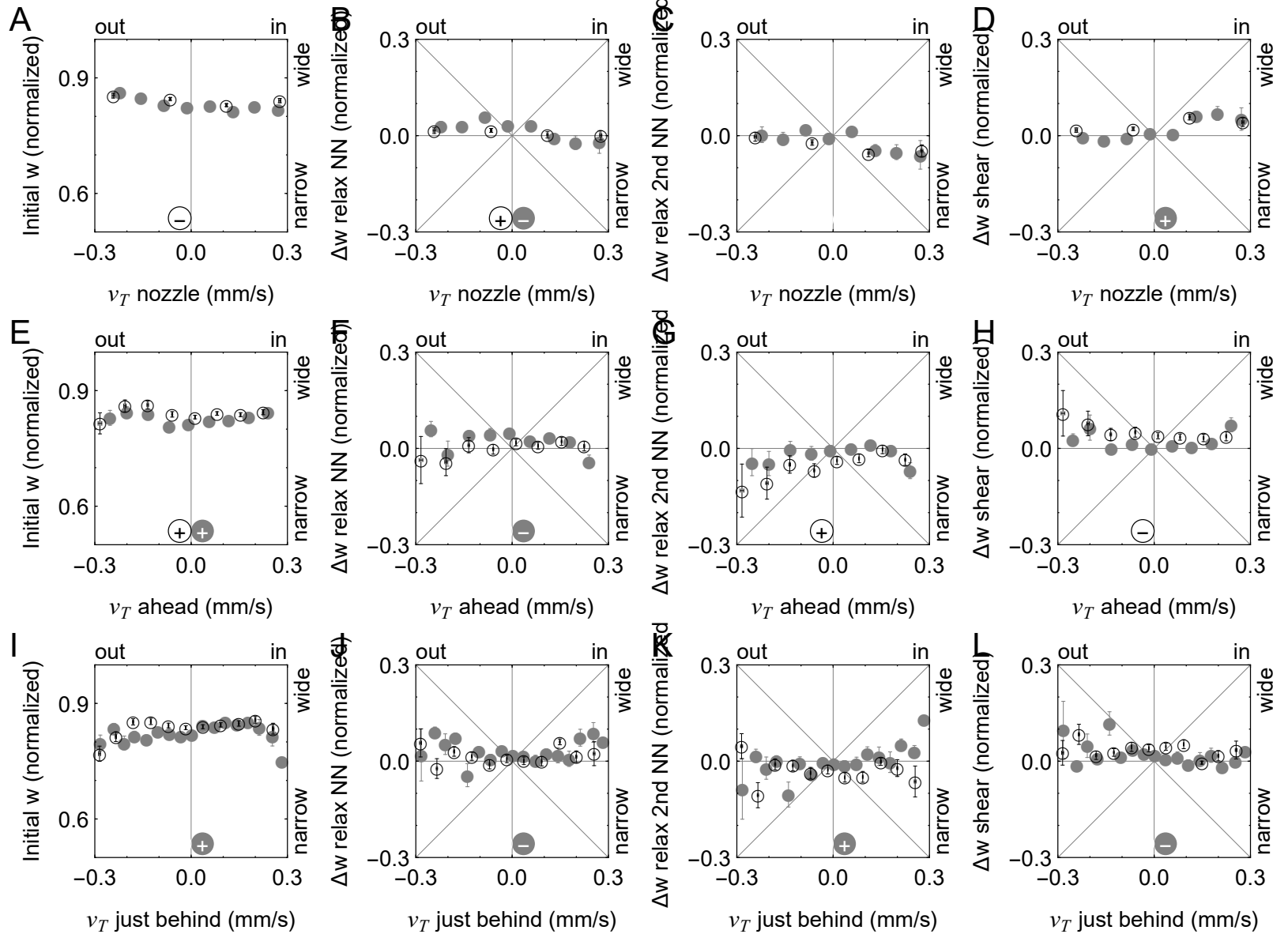

$v_{T}$ just behind $(\mathrm{mm} / \mathrm{s})$
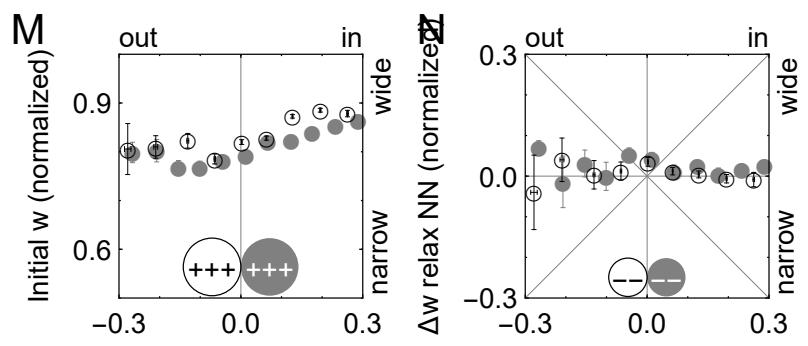

$v_{T}$ behind outer $(\mathrm{mm} / \mathrm{s})$

$v_{T}$ behind outer $(\mathrm{mm} / \mathrm{s})$
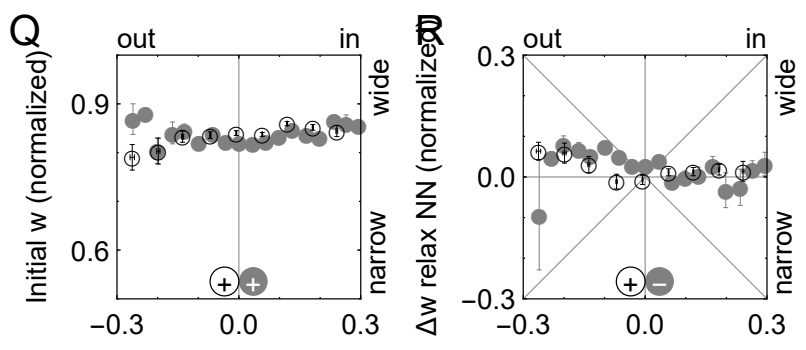

$v_{T}$ far behind $(\mathrm{mm} / \mathrm{s})$

$v_{T}$ far behind (mm/s)
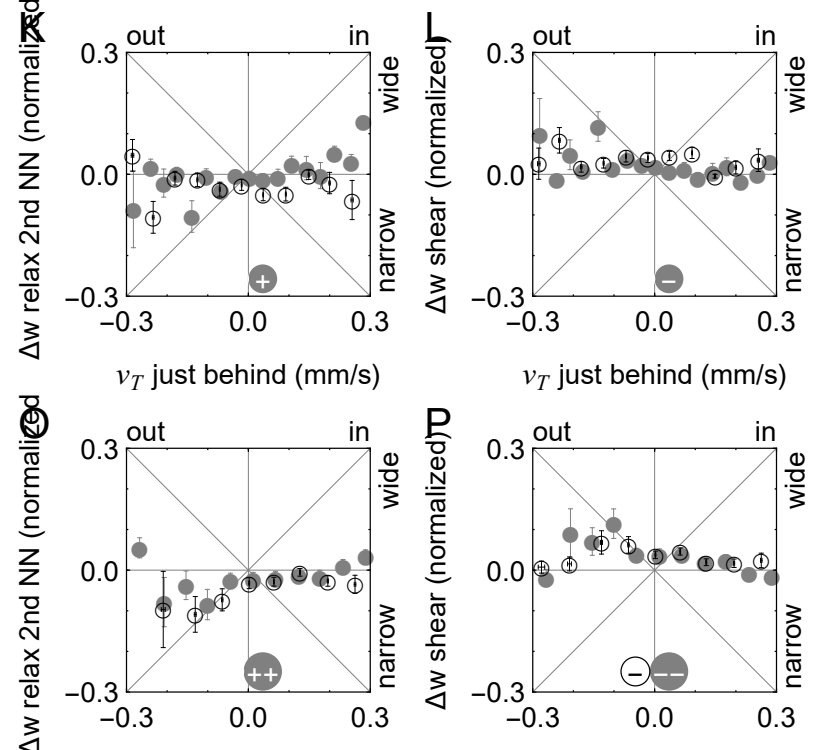

$v_{T}$ behind outer $(\mathrm{mm} / \mathrm{s})$

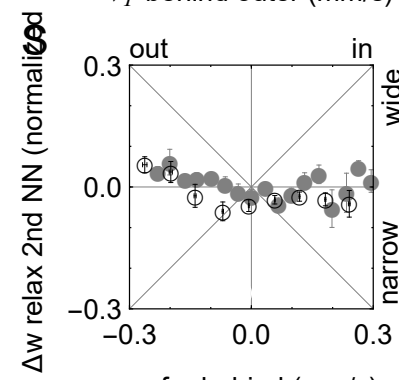

$v_{T}$ behind outer $(\mathrm{mm} / \mathrm{s})$

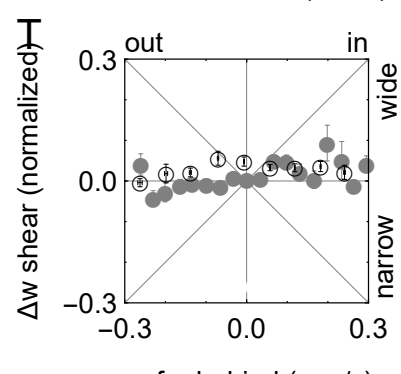

$v_{T}$ far behind $(\mathrm{mm} / \mathrm{s})$

$v_{T}$ far behind $(\mathrm{mm} / \mathrm{s})$

Figure S22: Summary of statistically significant Kendall $\tau$ correlations between transverse flow velocities and relative particle distribution positions and widths over the course of the print, where $p<0.05$. "-" indicates a negative correlation where $-0.1<\tau<0$, " ++ " indicates a stronger positive correlation where $0.1<\tau<0.2$, and so on, where $-1<\tau<1$. Error bars indicate standard error with respect to the line $y=0$. 


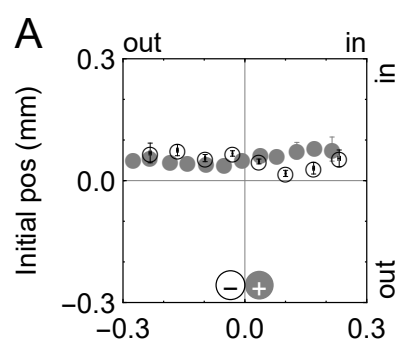

$v_{T}$ behind $\mathrm{NN}(\mathrm{mm} / \mathrm{s})$

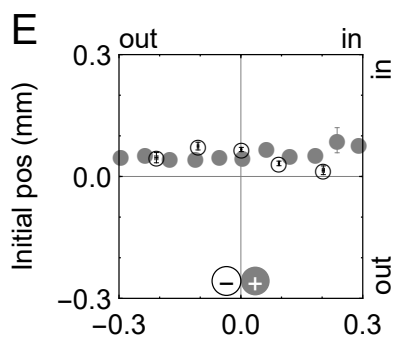

$v_{T}$ behind $2 \mathrm{NN}(\mathrm{mm} / \mathrm{s})$
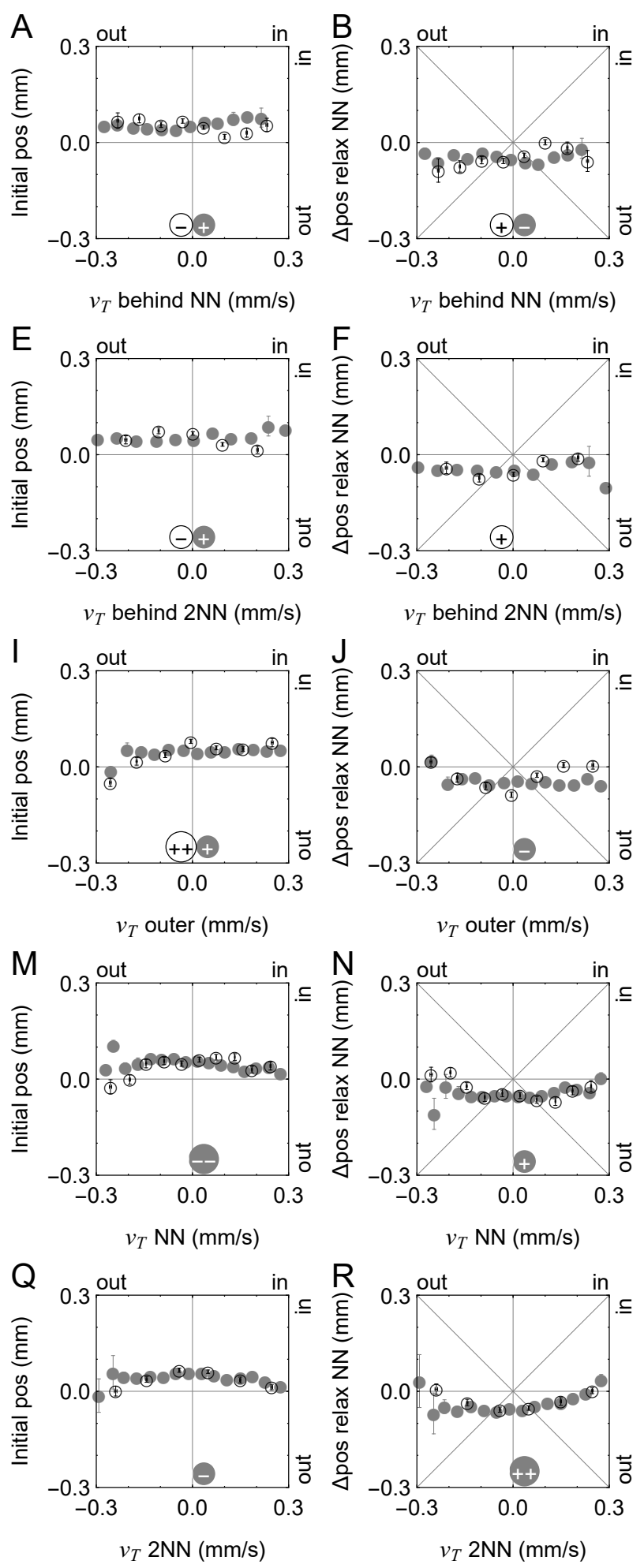

$v_{T}$ behind NN $(\mathrm{mm} / \mathrm{s})$

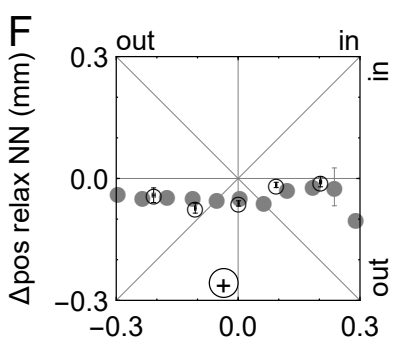

$v_{T}$ behind $2 \mathrm{NN}(\mathrm{mm} / \mathrm{s})$
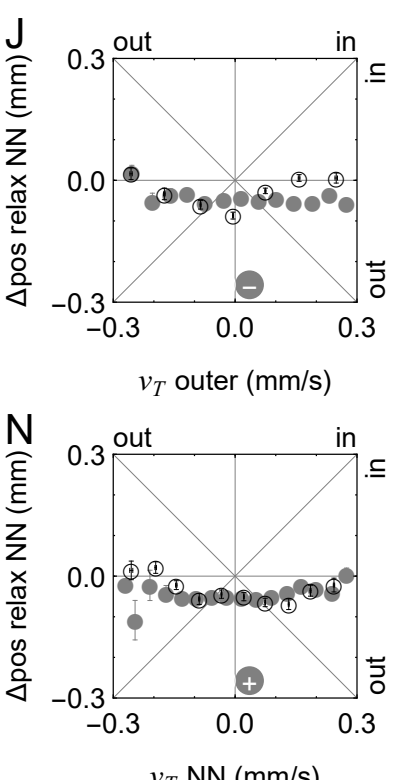
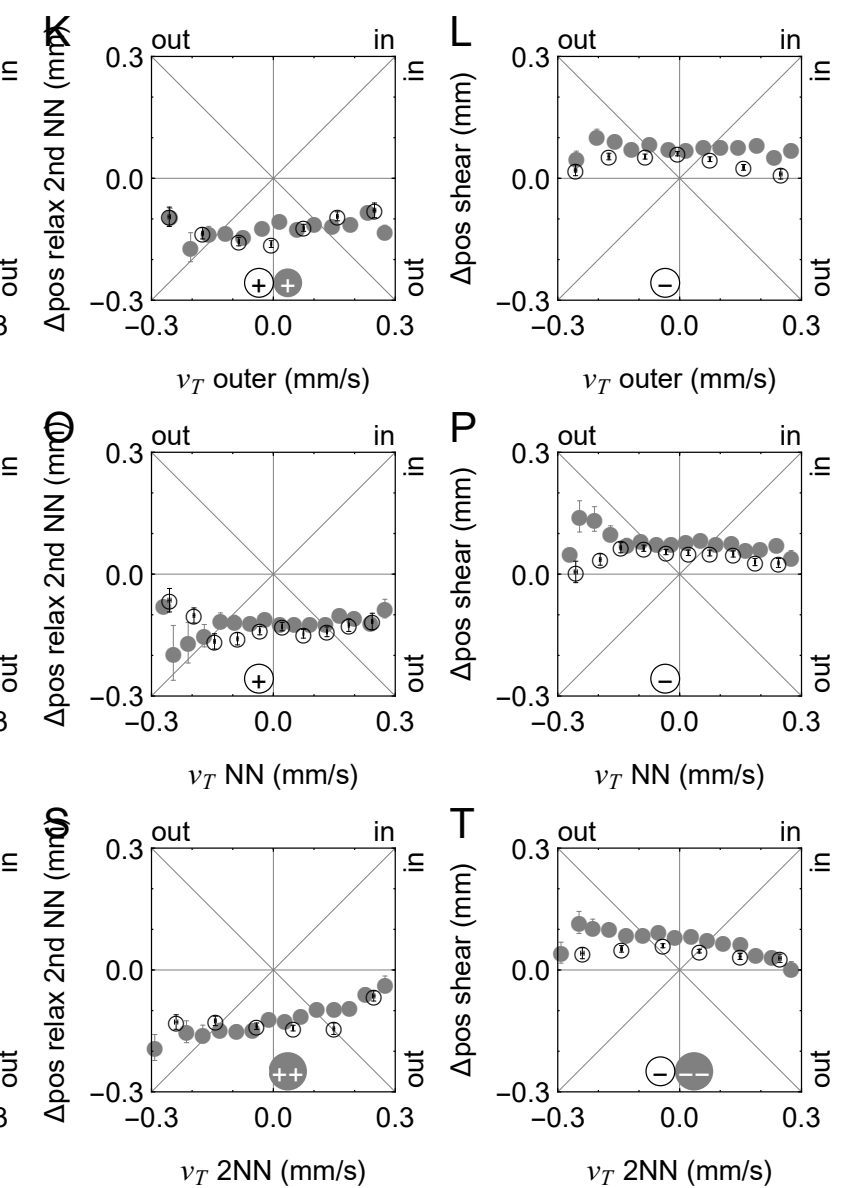

Figure S23: Summary of statistically significant Kendall $\tau$ correlations between transverse flow velocities and relative particle distribution positions and widths over the course of the print, where $p<0.05$. "-" indicates a negative correlation where $-0.1<\tau<0$, " ++ " indicates a stronger positive correlation where $0.1<\tau<0.2$, and so on, where $-1<\tau<1$. Error bars indicate standard error with respect to the line $y=0$. 

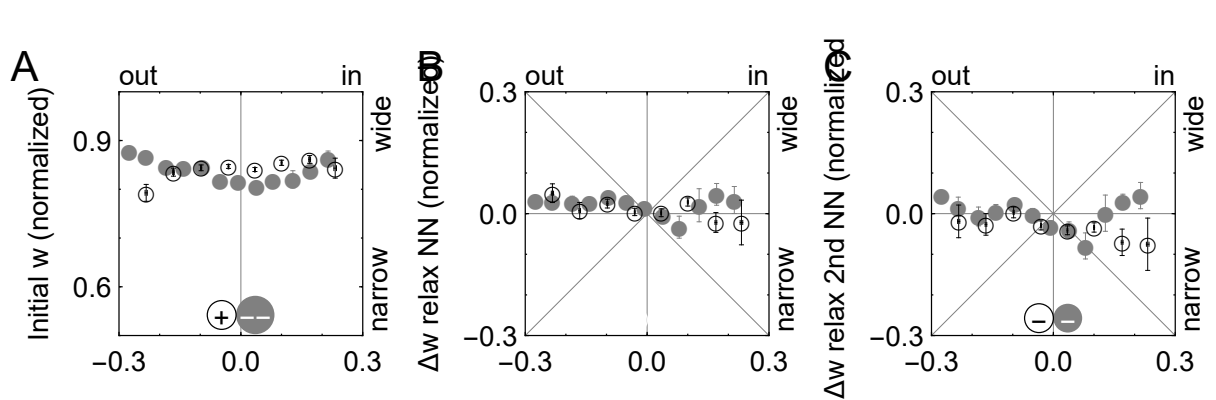

Layer-by-layer $\odot$ Bath
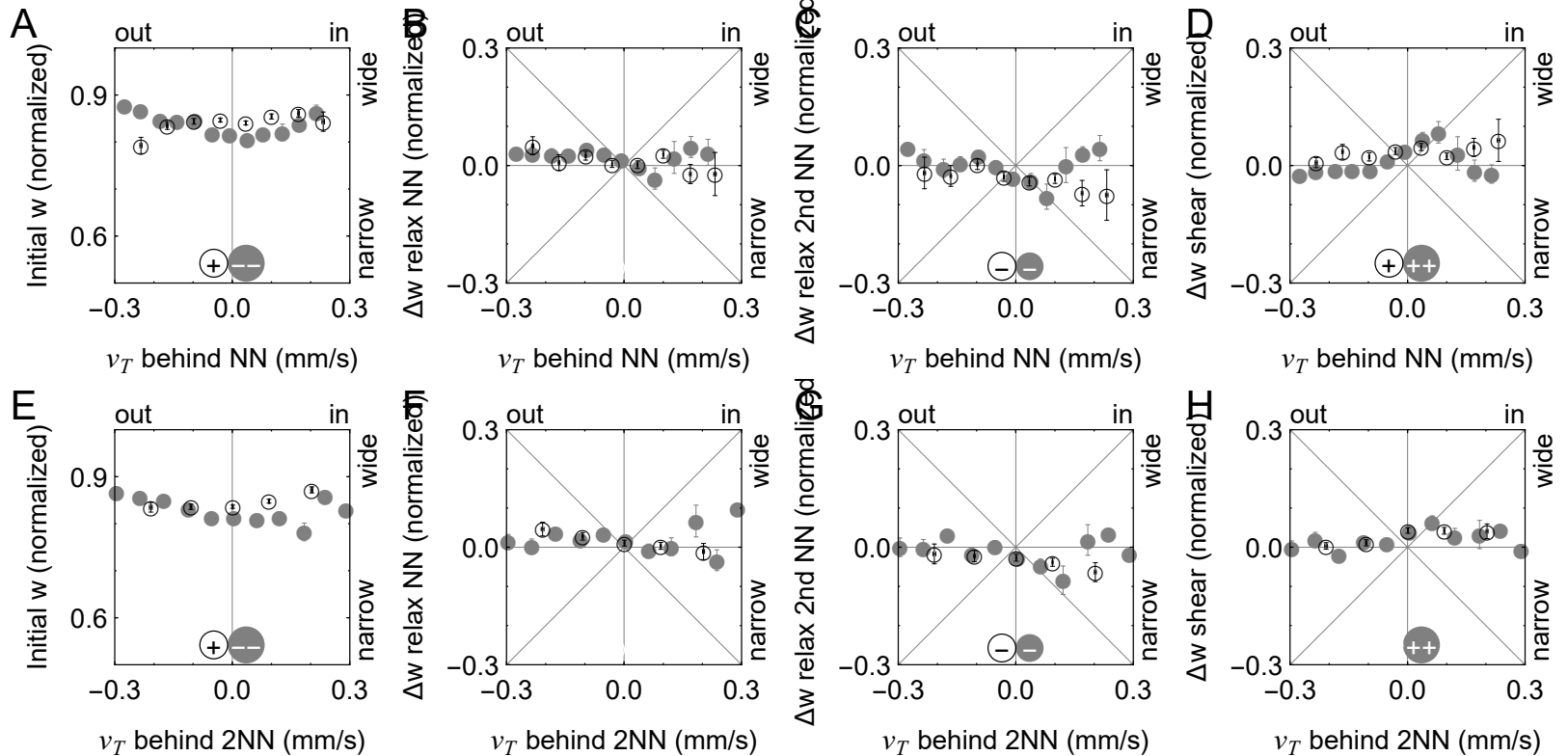

$v_{T}$ behind $2 \mathrm{NN}(\mathrm{mm} / \mathrm{s})$
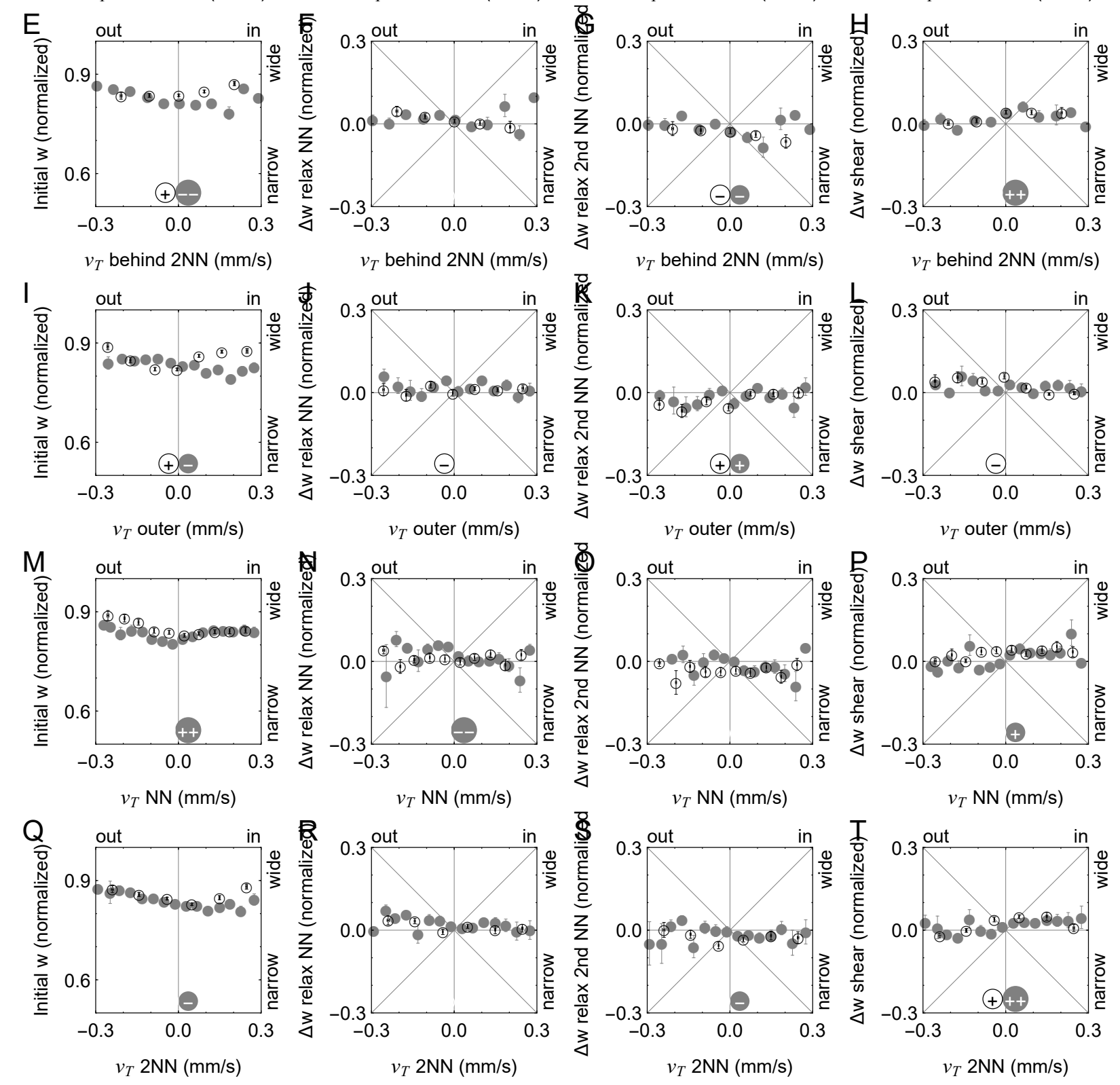

Figure S24: Summary of statistically significant Kendall $\tau$ correlations between transverse flow velocities and relative particle distribution positions and widths over the course of the print, where $p<0.05$. "-" indicates a negative correlation where $-0.1<\tau<0$, " ++ " indicates a stronger positive correlation where $0.1<\tau<0.2$, and so on, where $-1<\tau<1$. Error bars indicate standard error with respect to the line $y=0$. 
Table S20: Summary of statistically significant Kendall $\tau$ correlations between transverse flow velocities in different regions, where $p<0.05$.

"“" indicates a negative correlation where $-0.1<\tau<0$, "++" indicates a stronger positive correlation where $0.1<\tau<0.2$, and so on, where

$-1<\tau<1$.

\section{Layer-by-layer}

\section{nozitis}

nozzle

ahead

just behind

behind outer

far behind

behind NN

behind $2 \mathrm{NN}$

outer

$\mathrm{NN}$

2NN

Bath

nozzle

ahead

just behind

behind outer

far behind

behind NN

behind 2NN

outer

$\mathrm{NN}$

nozzle ahead just behind behind outer far behind behind NN behind 2NN outer NN

$2 \mathrm{NN}$

$2 \mathrm{NN}$

\begin{tabular}{|c|c|c|c|c|c|c|c|c|}
\hline++ & - & & & & + & + & + & + \\
\hline & - & + & & - & - & ++ & ++ & + \\
\hline & & + & ++++ & +++ & ++ & ++ & ++ & \\
\hline & & & ++ & + & + & ++ & + & ++ \\
\hline & & & & +++ & ++ & + & ++ & + \\
\hline & & & & & +++ & + & + & + \\
\hline & & & & & & + & + & ++ \\
\hline & & & & & & & + & $\begin{array}{c}+ \\
+++\end{array}$ \\
\hline+ & + & + & + & + & & + & ++ & + \\
\hline & - & + & + & - & - & ++ & +++ & \\
\hline & & ++ & +++ & ++ & + & + & ++ & ++ \\
\hline & & & + & - - & -- & ++ & + & - \\
\hline & & & & ++++ & ++ & ++ & ++ & ++ \\
\hline & & & & & ++++ & + & + & ++ \\
\hline & & & & & & + & + & $\begin{array}{c}++ \\
+\end{array}$ \\
\hline & & & & & & & & $\begin{array}{l}+ \\
++\end{array}$ \\
\hline
\end{tabular}




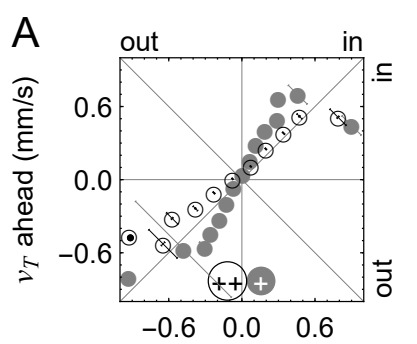

$v_{T}$ nozzle $(\mathrm{mm} / \mathrm{s})$

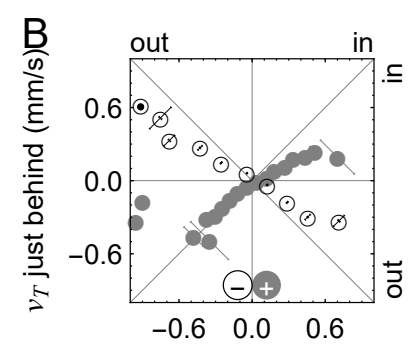

$v_{T}$ nozzle $(\mathrm{mm} / \mathrm{s})$

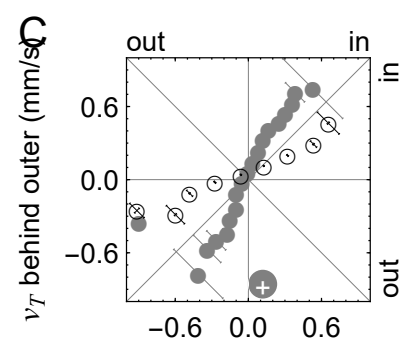

$v_{T}$ nozzle $(\mathrm{mm} / \mathrm{s})$

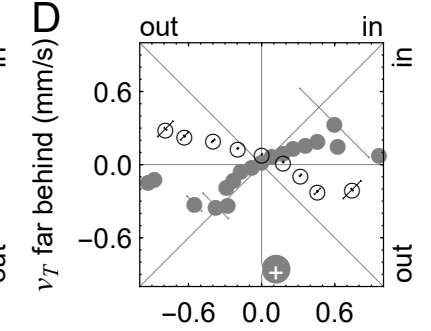

$v_{T}$ nozzle $(\mathrm{mm} / \mathrm{s})$

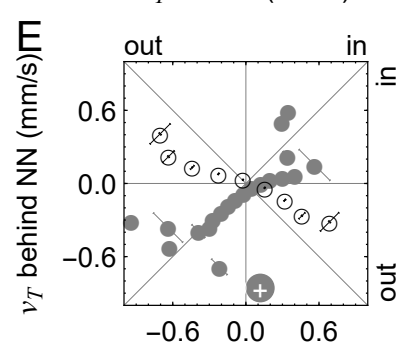

$v_{T}$ nozzle $(\mathrm{mm} / \mathrm{s})$

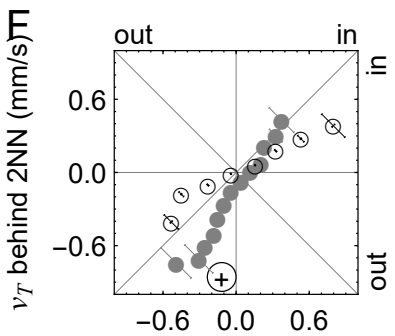

$v_{T}$ nozzle $(\mathrm{mm} / \mathrm{s})$

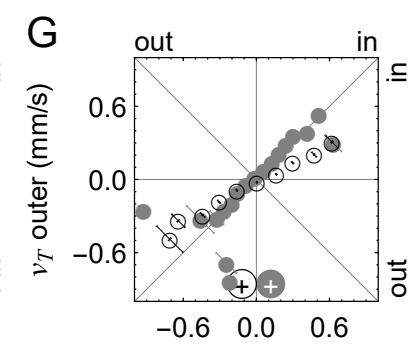

$v_{T}$ nozzle $(\mathrm{mm} / \mathrm{s})$
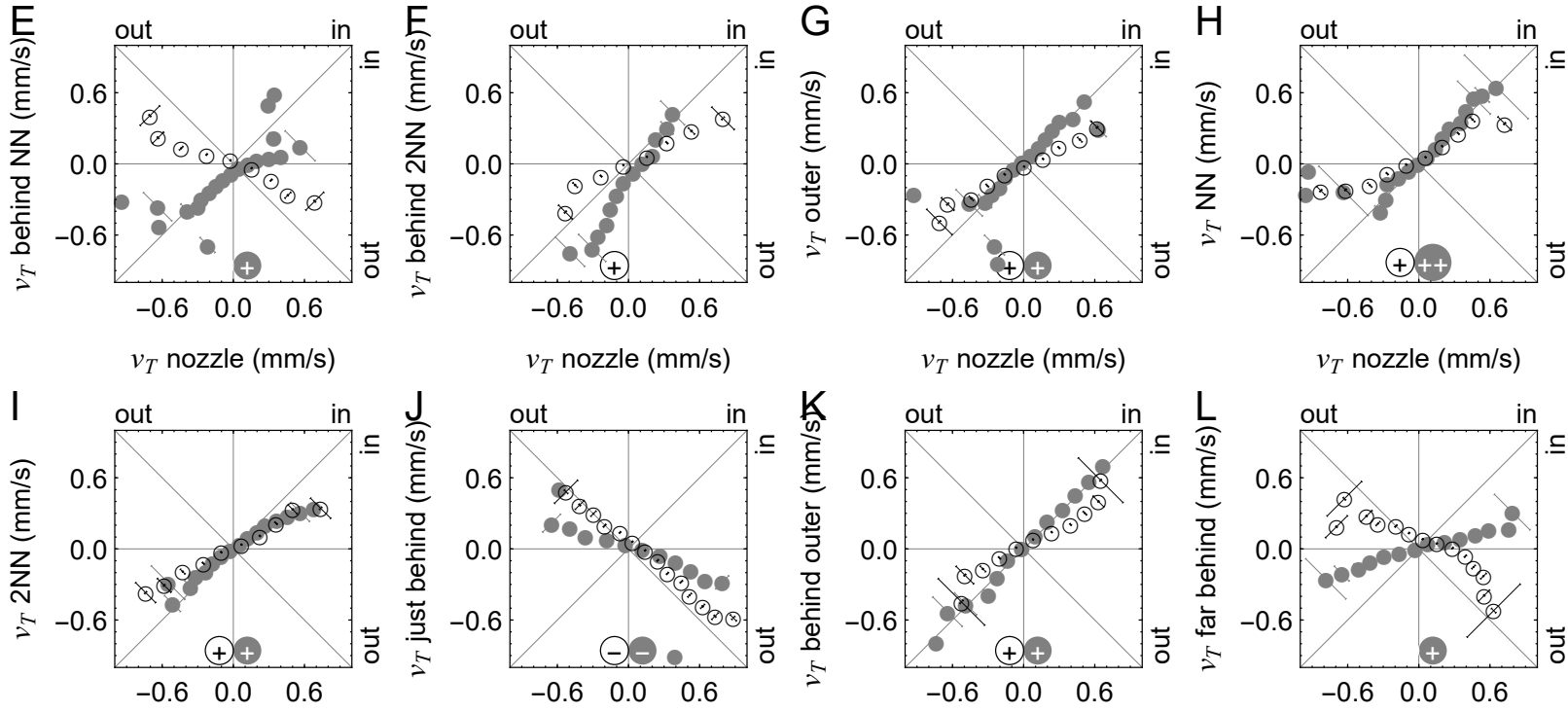

$v_{T}$ nozzle $(\mathrm{mm} / \mathrm{s})$ $v_{T}$ nozzle $(\mathrm{mm} / \mathrm{s})$

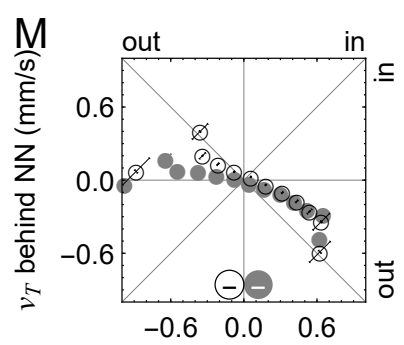

$v_{T}$ ahead $(\mathrm{mm} / \mathrm{s})$

$v_{T}$ ahead $(\mathrm{mm} / \mathrm{s})$

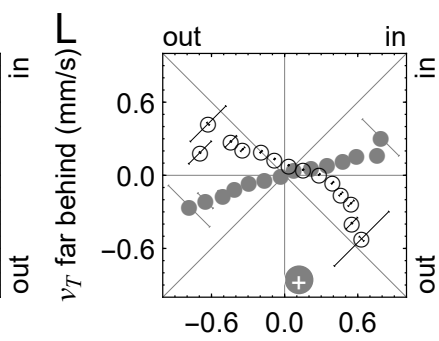

$v_{T}$ ahead $(\mathrm{mm} / \mathrm{s})$
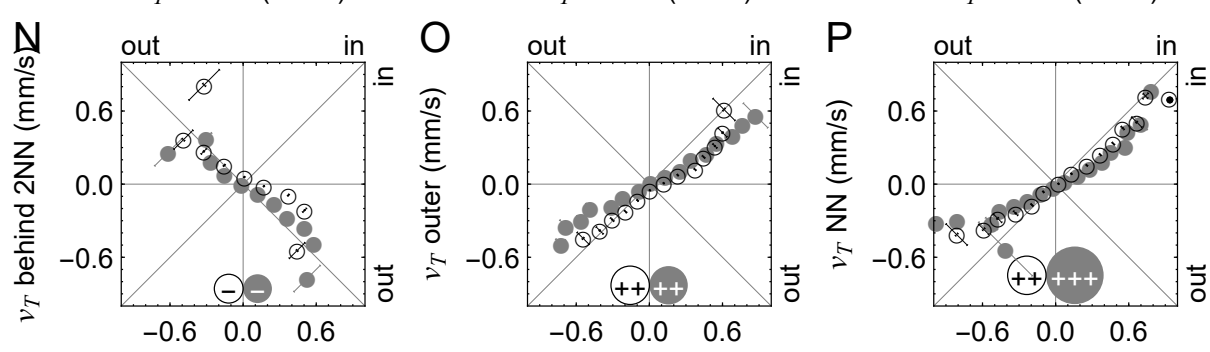

$v_{T}$ ahead $(\mathrm{mm} / \mathrm{s})$

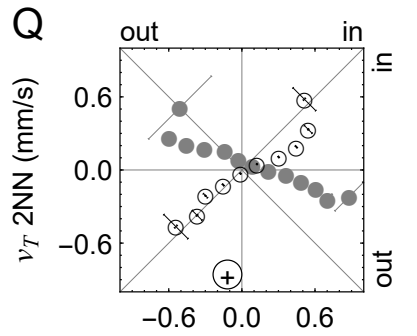

$v_{T}$ ahead $(\mathrm{mm} / \mathrm{s})$

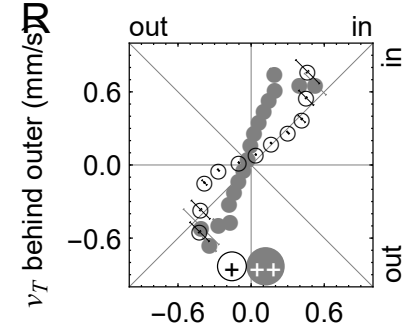

$v_{T}$ just behind $(\mathrm{mm} / \mathrm{s})$

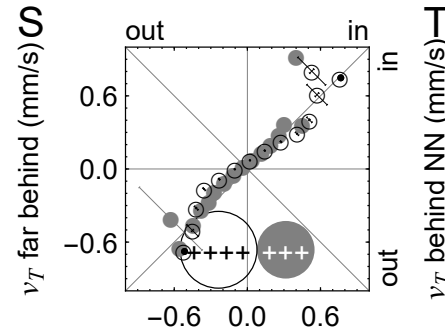

$v_{T}$ just behind $(\mathrm{mm} / \mathrm{s})$

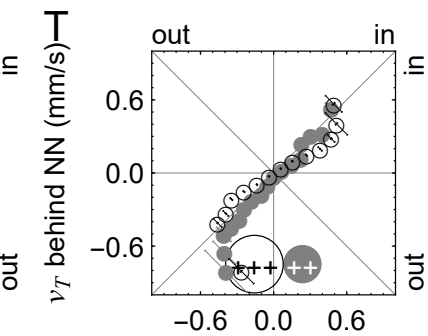

$v_{T}$ just behind $(\mathrm{mm} / \mathrm{s})$

Figure S25: Summary of statistically significant Kendall $\tau$ correlations between transverse flow velocities in different regions, where $p<0.05$. "-" indicates a negative correlation where $-0.1<\tau<0$, "++" indicates a stronger positive correlation where $0.1<\tau<0.2$, and so on, where $-1<\tau<1$. Error bars indicate standard error with respect to the line $-x=y$ or $x=y$. 


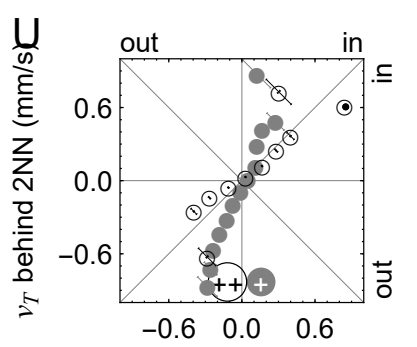

$v_{T}$ just behind $(\mathrm{mm} / \mathrm{s})$

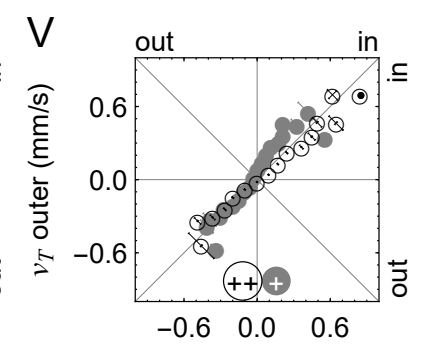

$v_{T}$ just behind $(\mathrm{mm} / \mathrm{s})$

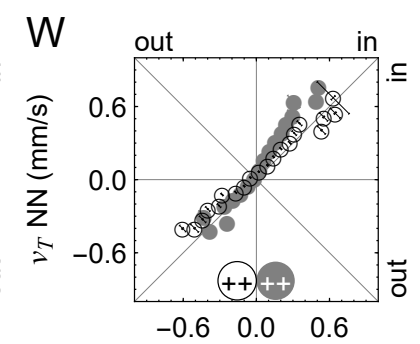

$v_{T}$ just behind $(\mathrm{mm} / \mathrm{s})$

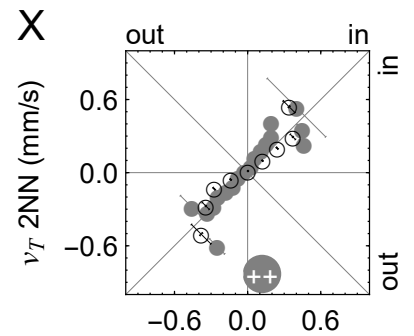

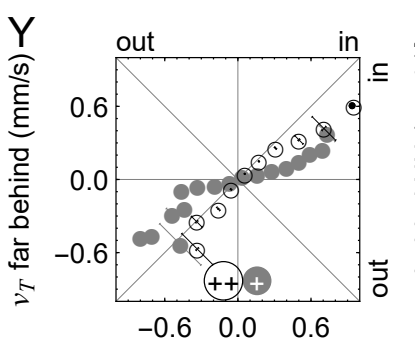

$v_{T}$ behind outer $(\mathrm{mm} / \mathrm{s})$

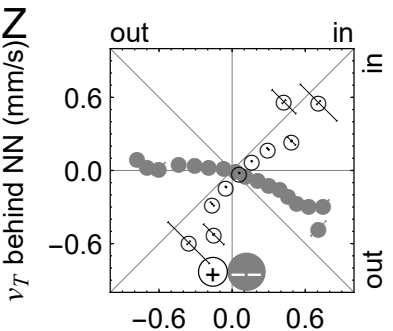

$v_{T}$ behind outer $(\mathrm{mm} / \mathrm{s})$

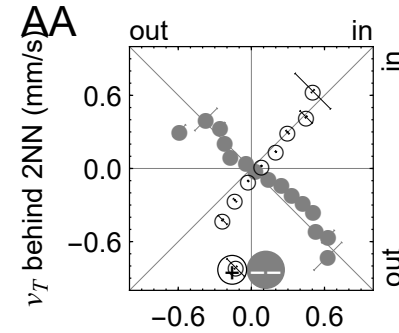

$v_{T}$ behind outer $(\mathrm{mm} / \mathrm{s})$

$v_{T}$ just behind $(\mathrm{mm} / \mathrm{s})$

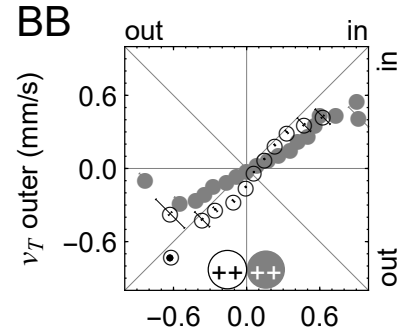

$v_{T}$ behind outer $(\mathrm{mm} / \mathrm{s})$

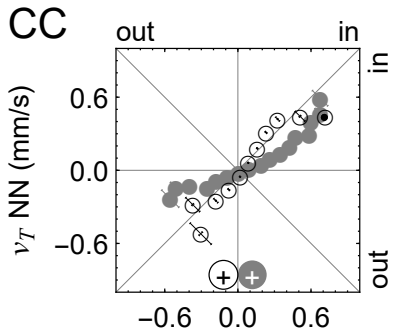

$v_{T}$ behind outer $(\mathrm{mm} / \mathrm{s})$
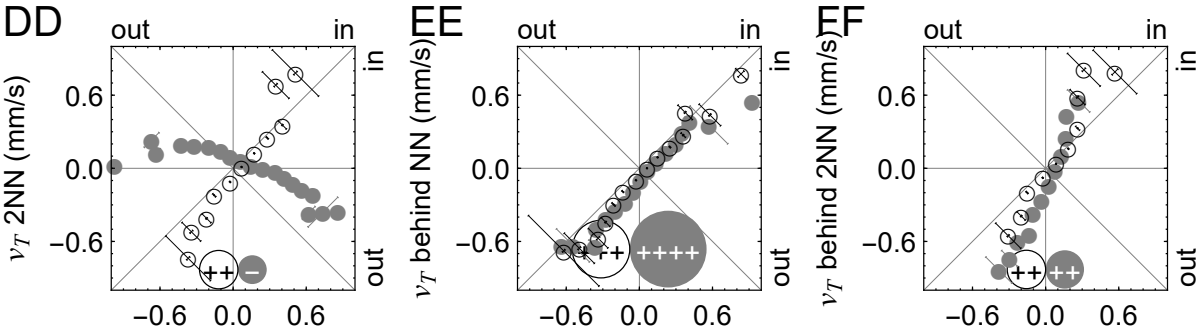

$v_{T}$ behind outer $(\mathrm{mm} / \mathrm{s})$
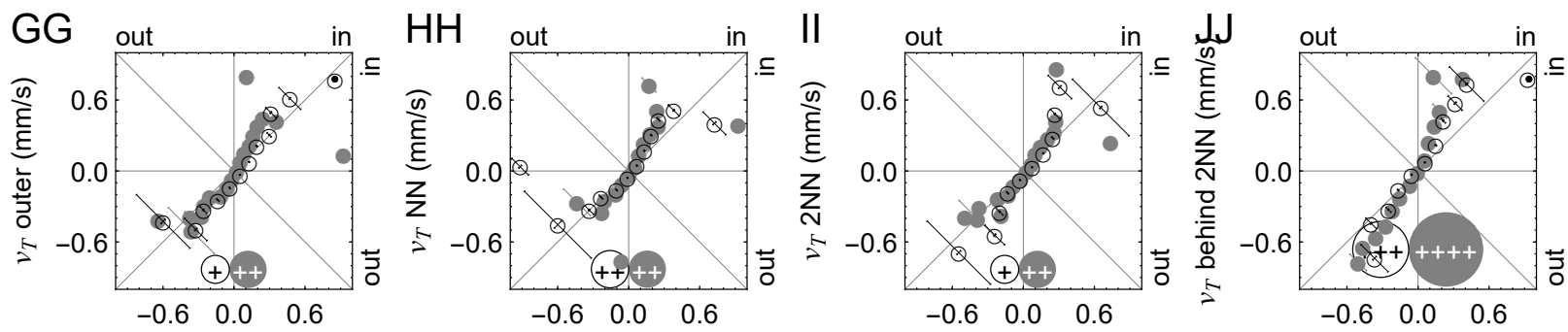

$v_{T}$ far behind $(\mathrm{mm} / \mathrm{s})$

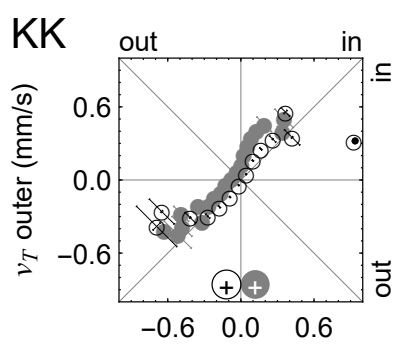

$v_{T}$ behind $\mathrm{NN}(\mathrm{mm} / \mathrm{s})$

$v_{T}$ far behind $(\mathrm{mm} / \mathrm{s})$

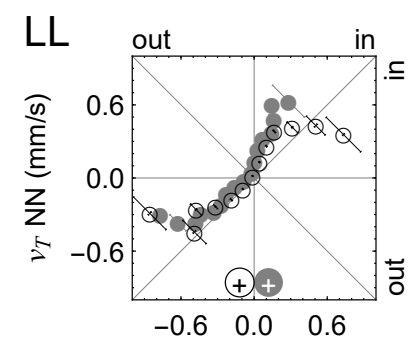

$v_{T}$ behind $\mathrm{NN}(\mathrm{mm} / \mathrm{s})$

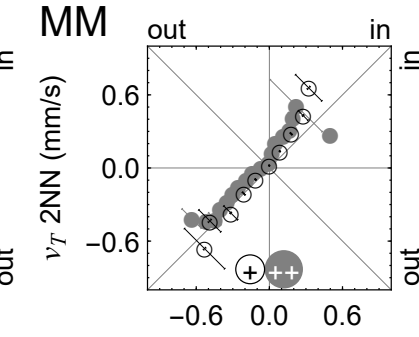

$v_{T}$ behind $\mathrm{NN}(\mathrm{mm} / \mathrm{s})$

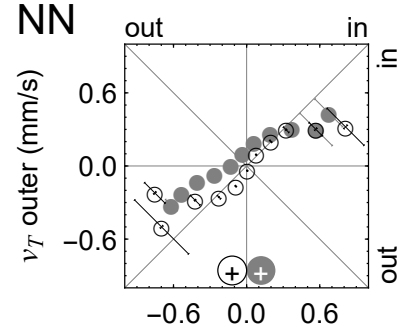

$v_{T}$ behind $2 \mathrm{NN}(\mathrm{mm} / \mathrm{s})$

Figure S26: Summary of statistically significant Kendall $\tau$ correlations between transverse flow velocities in different regions, where $p<0.05$. "-" indicates a negative correlation where $-0.1<\tau<0$, "++" indicates a stronger positive correlation where $0.1<\tau<0.2$, and so on, where $-1<\tau<1$. Error bars indicate standard error with respect to the line $-x=y$ or $x=y$. 


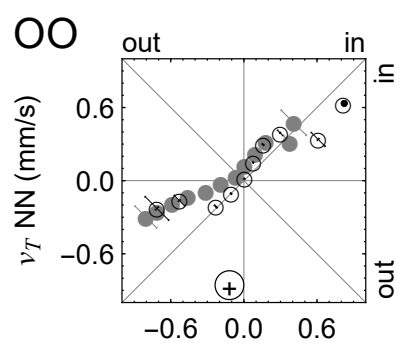

$v_{T}$ behind $2 \mathrm{NN}(\mathrm{mm} / \mathrm{s})$

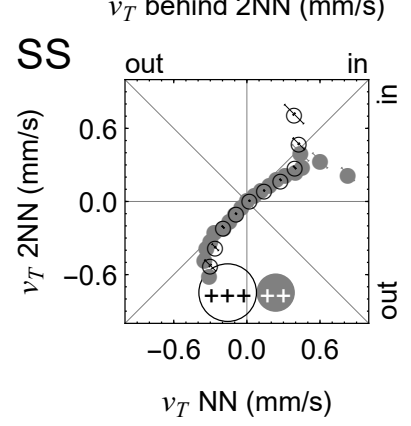

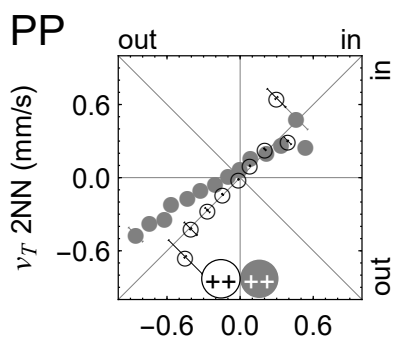

$v_{T}$ behind $2 \mathrm{NN}(\mathrm{mm} / \mathrm{s})$

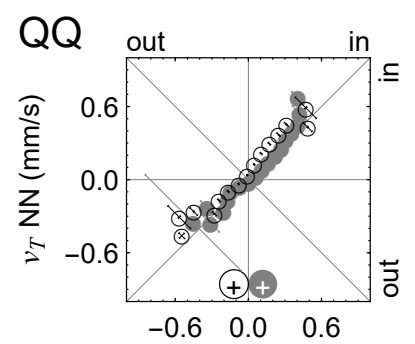

$v_{T}$ outer $(\mathrm{mm} / \mathrm{s})$

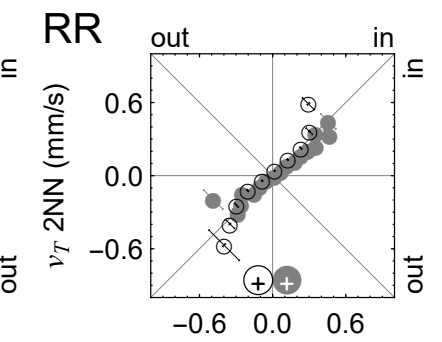

$v_{T}$ outer $(\mathrm{mm} / \mathrm{s})$ . 